\begin{tabular}{|c|l|}
\hline Title & PARA BOLIC GEOMETRIES A SSOCIA TED WITH DIFFERENTIAL EQUA TIONS OF FINITE TYPE \\
\hline Author(s) & Yamaguchi, Keizo; Yatsui, Tomoaki \\
\hline Citation & Hokkaido University Preprint Series in Mathematics, 756, 1-41 \\
\hline Issue Date & 2005 \\
\hline DOI & 10.14943/83906 \\
\hline Doc URL & http://hdl.handle.net/2115/69564 \\
\hline Type & bulletin (article) \\
\hline File Information & pre756.pdf \\
\hline
\end{tabular}

Instructions for use 


\title{
PARABOLIC GEOMETRIES \\ ASSOCIATED WITH \\ DIFFERENTIAL EQUATIONS OF FINITE TYPE
}

\author{
KEIZO YAMAGUCHI AND TOMOAKI YATSUI
}

\begin{abstract}
We present here classes of parabolic geometries arising naturally from Seashi's principle to form good classes of linear differential equations of finite type, which generalize the cases of second and third order ODE for scalar function. We will explicitly describe the symbols of these differential equations. The model equations of these classes admit nonlinear contact transformations and their symmetry algebras become finite dimensional and simple.
\end{abstract}

\section{INTRODUCTION}

The geometry of ordinary differential equations for scalar function is strongly linked to the Lie algebra $\mathfrak{s l}(2, \mathbb{R})=\mathfrak{s l}(\hat{V})$, where $\hat{V}$ is a vector space of dimension 2. Associated to the geometry of $k$-th order ordinary differential equation;

$$
\frac{d^{k} y}{d x^{k}}=F\left(x, y, \frac{d y}{d x}, \ldots, \frac{d^{k-1} y}{d x^{k-1}}\right)
$$

we have the irreducible representation of $\hat{\mathfrak{l}}=\mathfrak{s l}(\hat{V})$ on $S=S^{k-1}\left(\hat{V}^{*}\right)$, where $S^{k-1}\left(\hat{V}^{*}\right)$ is the space of homogeneopus polynomials of degree $k-1$ in two variables and is the solution space of the model equation $\frac{d^{k} y}{d x^{k}}=0$ on the model space $\mathbb{P}^{1}(\mathbb{R})=\mathbb{P}(\hat{V})$. It is known that the Lie algebra $\mathfrak{l}=\mathfrak{g l}(\hat{V})$ is the infinitesimal group of linear automorphisms of the model equation (cf. Proposition 4.4.1 [Sea88]). Moreover the Lie algebra $\mathfrak{g}^{k}=\mathfrak{g}^{k}(1,1)$ of infinitesimal contact transformations of $\frac{d^{k} y}{d x^{k}}=0$ is given as follows; (1) $\mathfrak{g}^{2}$ is isomorphic to $\mathfrak{s l}(3, \mathbb{R})$. (2) $\mathfrak{g}^{3}$ is isomorphic to $\mathfrak{s p}(2, \mathbb{R})$. (3) Otherwise, for $k \geqq 4, \mathfrak{g}^{k}=S \oplus \mathfrak{l}$ is a subalgebra of the affine Lie algebra $\mathfrak{A}(S)=S \oplus \mathfrak{g l}(S)$ (see $\S 2.1)$. The Lie algebra $\mathfrak{g}^{k}$ plays the fundamental role in the contact geometry of k-th order ordinary differential equations.

Thus, when $k=2$ and 3, special phenomena prevail and result in rich automorphism groups so that these two cases offer examples of parabolic geometries associated with differential equations. Here the Parabolic Geometry is a geometry modeled after the homogeneous space $G / P$, where $G$ is a (semi-)simple Lie group and $P$ is a parabolic subgroup of $G$ (cf. [Bai93]). Precisely, in this paper, we mean, by a parabolic geometry, the geometry associated with the simple graded Lie algebra in the sense of N.Tanaka ([Tan79]). The main purpose of this paper is to seek to find other such special phenomena and to present other classes of parabolic geometries associated with differential equations of finite type, which naturally arise from Se-ashi's principle and generalize the above cases of $\mathfrak{g}^{2}$ and $\mathfrak{g}^{3}$.

For the geometry of differential equations of finite type, our study is based on the geometry of differential systems in the following way (cf. [YY02]): We regard a $k$-th order differential equation as a submanifold $R$ of the $k$-jet space $J^{k}(n, m)$ for $n$ independent and $m$ dependent variables. Defined on $R$, we have the differential system $\hat{D}$ obtained by restricting to $R$ the canonical system $C^{k}$ on $J^{k}(n, m)$ (see $\S 2.1$ ). Especially, when 
$R$ is a $k$-th order involutive differential equation of finite type, $p=\left.\pi_{k-1}^{k}\right|_{R}: R \rightarrow J^{k-1}$ is an immersion so that we have a pseudo-product structure $D=E \oplus F$ on $R$, where $D$ is the pullback $\left(p_{*}\right)^{-1}\left(C^{k-1}\right)$ of $C^{k-1}$ through $p, E$ is the restriction of $C^{k}$ to $R$ and $F=\operatorname{Ker}\left(\left.\pi_{k-2}^{k}\right|_{R}\right)_{*}$ is the fibre direction of $\left.\pi_{k-2}^{k}\right|_{R}$.

Now, let us recall Se-ashi's procedure to form good classes of linear differential equations of finite type, following [Sea88] and [YY02]. Se-ashi's procedure starts from a reductive graded Lie algebra (GLA) $\mathfrak{l}=\mathfrak{l}_{-1} \oplus \mathfrak{l}_{0} \oplus \mathfrak{l}_{1}$ and a faithful irreducible $\mathfrak{l}$-module $S$. Then we form the pseudo-product GLA $\mathfrak{g}=\bigoplus_{p \in \mathbb{Z}} \mathfrak{g}_{p}$ of type $(\mathfrak{l}, S)$ as follows: Let $\mathfrak{l}=\mathfrak{l}_{-1} \oplus \mathfrak{l}_{0} \oplus \mathfrak{l}_{1}$ be a finite dimensional reductive GLA of the first kind such that

(1) The ideal $\hat{\mathfrak{l}}=\mathfrak{l}_{-1} \oplus\left[\mathfrak{l}_{-1}, \mathfrak{l}_{1}\right] \oplus \mathfrak{l}_{1}$ of $\mathfrak{l}$ is a simple Lie algebra.

(2) The center $\mathfrak{z}(\mathfrak{l})$ of $\mathfrak{l}$ is contained in $\mathfrak{l}_{0}$.

Let $S$ be a finite dimensional faithful irreducible $\mathfrak{l}$-module. We put

$$
S_{-1}=\left\{s \in S \mid \mathfrak{l}_{1} \cdot s=0\right\}
$$

and

$$
S_{p}=\operatorname{ad}\left(\mathfrak{l}_{-1}\right)^{-p-1} S_{-1} \text { for } p<0
$$

We form the semi-direct product $\mathfrak{g}$ of $\mathfrak{l}$ by $S$, and put

$$
\begin{gathered}
\mathfrak{g}=S \oplus \mathfrak{l}, \quad[S, S]=0 \\
\mathfrak{g}_{k}=\mathfrak{l}_{k}(k \geqq 0), \quad \mathfrak{g}_{-1}=\mathfrak{l}_{-1} \oplus S_{-1}, \\
\mathfrak{g}_{p}=S_{p}(p<-1) .
\end{gathered}
$$

Then $\mathfrak{g}=\bigoplus_{p \in \mathbb{Z}} \mathfrak{g}_{p}$ enjoys the following properties (Lemma 2.1);

(1) $S=\bigoplus_{p=-1}^{-\mu} S_{p}$, where $S_{-\mu}=\left\{s \in S \mid\left[\mathfrak{l}_{-1}, s\right]=0\right\}$.

(2) $\mathfrak{m}=\bigoplus_{p<0} \mathfrak{g}_{p}$ is generated by $\mathfrak{g}_{-1}$.

(3) $S_{p}$ is naturally embedded as a subspace of $W \otimes S^{\mu+p}\left(\mathfrak{l}_{-1}{ }^{*}\right)$

through the bracket operation in $\mathfrak{m}$, where $W=S_{-\mu}$.

Thus $S=S_{-\mu} \oplus S_{-\mu+1} \oplus \cdots \oplus S_{-1} \subset W \oplus W \otimes V^{*} \oplus \cdots \oplus W \otimes S^{\mu-1}\left(V^{*}\right)$ defines a symbol of $\mu$-th order differential equations of finite type by putting $S_{0}=\{0\} \subset W \otimes S^{\mu}\left(V^{*}\right)$. We can construct the model linear equation $R_{o}$ of finite type, whose symbol at each point is isomorphic to $S$ (see $\S 4$ [Sea88]). $R_{o}$ is a $\mu$-th order involutive differential equation of finite type. Then, we see that the symbol algebra of $\left(R_{o}, D_{o}\right)$ is isomorphic to $\mathfrak{m}$, where $D_{o}$ is the pullback of the canonical system $C^{\mu-1}$ on the $(\mu-1)$-jet space $J^{\mu-1} \cdot \mathfrak{m}$ has the splitting $\mathfrak{g}_{-1}=\mathfrak{l}_{-1} \oplus S_{-1}$, corresponding to the pseudo-product structure on $R_{o}$, where $V=\mathfrak{l}_{-1}$ and $W=S_{-\mu}$. In this way, $\mathfrak{m}$ is a symbol algebra of $\mu$-th order differential equation of finite type, which is called the typical symbol of type $(\mathfrak{l}, S)$.

This class of higher order (linear) differential equations of finite type were first appeared in the work of Y.Se-ashi [Sea88], who discussed the linear equivalence of this class of equations and gave the complete system of differential invariants of these equations, generalizing the classical theory of Laguerre-Forsyth for linear ordinary differential equations.

We ask the following question for the pseudo-product GLA $\mathfrak{g}=\bigoplus_{p \in \mathbb{Z}} \mathfrak{g}_{p}$ of type $(\mathfrak{l}, S)$ : When is $\mathfrak{g}$ the prolongation of $\mathfrak{m}$ or $\left(\mathfrak{m}, \mathfrak{g}_{0}\right)$ ?

Namely we ask whether $\mathfrak{g}$ exhausts all the infinitesimal automorphisms of the differential system $\left(R_{o}, D_{o}\right)$ or its psedo-product structure.

The answer to this question is given in Theorem 5.2 of [YY02] (Theorem 2.1 below), where we can find the classes of parabolic geometries, which generalize the cases of second and third order ordinary differential equations. More precisely, this Theorem states : For a 
pseudo-product $G L A \mathfrak{g}=\bigoplus_{p=-\mu}^{1} \mathfrak{g}_{p}$ of type $(\mathfrak{l}, S)$ satisfying the condition $H^{1}(\mathfrak{m}, \mathfrak{g})_{0,0}=0$, $\mathfrak{g}$ is the prolongation of $\mathfrak{m}=\bigoplus_{p<0} \mathfrak{g}_{p}$ except for three cases. Let $\check{\mathfrak{b}}=\bigoplus_{p \in \mathbb{Z}} \check{\mathfrak{b}}_{p}$ be the prolongation of $\mathfrak{g}=\mathfrak{b}_{-1} \oplus \mathfrak{b}_{0}$, where $\mathfrak{b}_{-1}=S$ and $\mathfrak{b}_{0}=\mathfrak{l}$. Then the three exceptional cases correspond to cases : (a) $\operatorname{dim} \check{\mathfrak{b}}<\infty$ and $\check{\mathfrak{b}}_{1} \neq 0,(b) \operatorname{dim} \check{\mathfrak{b}}=\infty,(c)$ g is a pseudo-projective GLA (for the detail, see $\S 2$ ). In case $(a), \check{\mathfrak{b}}=\mathfrak{b}_{-1} \oplus \mathfrak{b}_{0} \oplus \check{\mathfrak{b}}_{1}$ becomes a simple graded Lie algebra containing $\mathfrak{g}=\mathfrak{b}_{-1} \oplus \mathfrak{b}_{0}$ as a parabolic subalgebra. Thus, basically, the case $(a)$ corresponds to parabolic geometries, we are seeking. In fact, in the case of $k$-th order ordinary differential equations for scalar function, $\mathfrak{g}^{2}$ and $\mathfrak{g}^{3}$ belong to case $(a)$ and $\mathfrak{g}^{k}$ belongs to case $(c)$ for $k \geqq 4$.

In $\S 2$, we will recall the above results from [YY02]. The symbol algebras of these parabolic geometries will be given in Theorem 2.1 in terms of root space decompositions of the corresponding simple Lie algebras. We will describe these symbol algebras and the model differential equations of finite type explicitly by utilizing the explicit matrices description of the simple graded Lie algebra $\check{\mathfrak{b}}$ for the classical cases in $\S 3$ and by describing the structure of $\mathfrak{m}$ explicitly by use of the Chevalley basis of the exceptional simple Lie algebras in $\S 4$. Finally, in $\S 5$, we will discuss about the equivalence of each parabolic geometry associated with the differential equations of finite type explicitly described in previous sections.

\section{Pseudo-Product GLA $\mathfrak{g}=\bigoplus_{p \in \mathbb{Z}} \mathfrak{g}_{p}$ OF TyPe $(\mathfrak{l}, S)$}

In this section, we will summarize the results in [YY02] and explain the prolongation theorem (Theorem 2.1). We will first discuss the prolongation of symbol algebras of the pseudo-product structures associated with higher order differential equations of finite type. Moreover we will generalize this algebra to the notion of the pseudo-product GLA (graded Lie algebras) of irreducible type and introduce the pseudo-product GLA $\mathfrak{g}=\bigoplus_{p \in \mathbb{Z}} \mathfrak{g}_{p}$ of type $(\mathfrak{l}, S)$ and ask when $\mathfrak{g}$ is the prolongation of $\mathfrak{m}$ or $\left(\mathfrak{m}, \mathfrak{g}_{0}\right)$, where $\mathfrak{m}=\bigoplus_{p<0} \mathfrak{g}_{p}$. In the answer to this question, we will find the classes of finite type differential equations mentioned in the introduction.

2.1. Pseudo-projective GLA of order $k$ of bidegree $(n, m)$. We first consider a system of higher order differential equations of finite type of the following form :

$$
\begin{aligned}
& \frac{\partial^{k} y^{\alpha}}{\partial x_{i_{1}} \cdots \partial x_{i_{k}}}=F_{i_{1} \cdots i_{k}}^{\alpha}\left(x_{1}, \ldots, x_{n}, y^{1}, \cdots, y^{m}, \ldots, p_{i}^{\beta}, \ldots, p_{j_{1} \cdots j_{k-1}}^{\beta}\right) \\
& \quad\left(1 \leqq \alpha \leqq m, 1 \leqq i_{1} \leqq \cdots \leqq i_{k} \leqq n\right)
\end{aligned}
$$

where $p_{i_{1} \cdots i_{\ell}}^{\beta}=\frac{\partial^{\ell} y^{\beta}}{\partial x_{i_{1}} \cdots \partial x_{i_{\ell}}}$. These equations define a submanifold $R$ in $k$-jets space $J^{k}$ such that the restriction $p$ to $R$ of the bundle projection $\pi_{k-1}^{k}: J^{k} \rightarrow J^{k-1}$ gives a diffeomorphism ;

$$
p: R \rightarrow J^{k-1} ; \text { diffeomorphism }
$$

On $J^{k}$, we have the Contact (differential) system $C^{k}$ defined by

$$
C^{k}=\left\{\varpi^{\alpha}=\varpi_{i}^{\alpha}=\cdots=\varpi_{i_{1} \cdots i_{k-1}}^{\alpha}=0\right\},
$$


where

$$
\left\{\begin{array}{l}
\varpi^{\alpha}=d y^{\alpha}-\sum_{i=1}^{n} p_{i}^{\alpha} d x_{i}, \quad(1 \leqq \alpha \leqq m) \\
\varpi_{i}^{\alpha}=d p_{i}^{\alpha}-\sum_{j=1}^{n} p_{i j}^{\alpha} d x_{j}, \quad(1 \leqq \alpha \leqq m, 1 \leqq i \leqq n) \\
\cdots \cdots \cdots \cdots, \\
\varpi_{i_{1} \cdots i_{k-1}}^{\alpha}=d p_{i_{1} \cdots i_{k-1}}^{\alpha}-\sum_{j=1}^{n} p_{i_{1} \cdots i_{k-1} j}^{\alpha} d x_{j} \\
\quad\left(1 \leqq \alpha \leqq m, 1 \leqq i_{1} \leqq \cdots \leqq i_{k-1} \leqq n\right) .
\end{array}\right.
$$

Then $C^{k}$ gives a foliation on $R$ when $R$ is integrable. Namely the restriction $E$ of $C^{k}$ to $R$ is completely integrable.

Thus, through the diffeomorphism (2.1), $R$ defines a completely integrable differential system $E^{\prime}=p_{*}(E)$ on $J^{k-1}$ such that

$$
C^{k-1}=E^{\prime} \oplus F^{\prime}, \quad F^{\prime}=\operatorname{Ker}\left(\pi_{k-2}^{k-1}\right)_{*}
$$

where $\pi_{k-2}^{k-1}: J^{k-1} \rightarrow J^{k-2}$ is the bundle projection. The triplet $\left(J^{k-1} ; E^{\prime}, F^{\prime}\right)$ is called the pseudo-product structure associated with $R$.

Corresponding to the splitting $D=E \oplus F=\left(p^{-1}\right)_{*}\left(C^{k-1}\right)$, we have the splitting in the symbol algebra of the regular differential system $(R, D) \cong\left(J^{k-1}, C^{k-1}\right)$ of type $\mathfrak{C}^{k-1}(n, m)$;

$$
\mathfrak{C}_{-1}=\mathfrak{e} \oplus \mathfrak{f},
$$

where $\mathfrak{e}=V, \mathfrak{f}=W \otimes S^{k-1}\left(V^{*}\right)$. At each point $x \in R, \mathfrak{e}$ corresponds to $E(x)$ (the point in $R^{(1)}$ over $x$ ) and $\mathfrak{f}$ corresponds to $\operatorname{Ker}\left(\pi_{k-2}^{k-1}\right)_{*}(p(x))$. Here we recall (see $\S 1.3[Y Y 02]$ for detail) that the fundamental graded Lie algebra (FGLA) $\mathfrak{C}^{k-1}(n, m)$ is defined by

$$
\mathfrak{C}^{k-1}(n, m)=\mathfrak{C}_{-k} \oplus \cdots \oplus \mathfrak{C}_{-2} \oplus \mathfrak{C}_{-1},
$$

where $\mathfrak{C}_{-k}=W, \mathfrak{C}_{p}=W \otimes S^{k+p}\left(V^{*}\right), \mathfrak{C}_{-1}=V \oplus W \otimes S^{k-1}\left(V^{*}\right)$. Here $V$ and $W$ are vector spaces of dimension $n$ and $m$ respectively and the bracket product of $\mathfrak{C}^{k-1}(n, m)=$ $\mathfrak{C}^{k-1}(V, W)$ is defined accordingly through the pairing between $V$ and $V^{*}$ such that $V$ and $W \otimes S^{k-1}\left(V^{*}\right)$ are both abelian subspaces of $\mathfrak{C}_{-1}$. Here $S^{r}\left(V^{*}\right)$ denotes the $r$-th symmetric product of $V^{*}$.

Now we put

$$
\check{\mathfrak{g}}_{0}=\left\{X \in \mathfrak{g}_{0}\left(\mathfrak{C}^{k-1}(n, m)\right) \mid[X, \mathfrak{e}] \subset \mathfrak{e},[X, \mathfrak{f}] \subset \mathfrak{f}\right\}
$$

and consider the (algebraic) prolongation $\mathfrak{g}^{k}(n, m)$ of $\left(\mathfrak{C}^{k-1}(n, m), \check{\mathfrak{g}}_{0}\right)$, which is called the pseudo-projective GLA of order $k$ of bidegree $(n, m)$ ([Tan89]). Here $\mathfrak{g}_{0}\left(\mathfrak{C}^{k-1}(n, m)\right)$ denotes the Lie algebra of gradation preserving derivations of $\mathfrak{C}^{k-1}(n, m)$.

Let $\check{G}_{0} \subset G L\left(\mathfrak{C}^{k-1}(n, m)\right)$ be the (gradation preserving) automorphism group of $\mathfrak{C}^{k-1}(n, m)$ which also preserve the splitting $\mathfrak{C}_{-1}=\mathfrak{e} \oplus \mathfrak{f}$. Then $\check{G}_{0}$ is the Lie subgroup of $G L\left(\mathfrak{C}^{k-1}(n, m)\right)$ with Lie algebra $\check{\mathfrak{g}}_{0}$. The pseudo-product structure on a $k$-th order differential equation $R$ of finite type given above, which is called the pseudo-projective system of order $k$ of bidegree $(n, m)$ in [Tan89], can be formulated as the $\check{G}_{0}^{\sharp}$-structure over a regular differential system of type $\mathfrak{C}^{k-1}(n, m)$ ([Tan70], [Tan89],[DKM99]). Thus the prolongation $\mathfrak{g}^{k}(n, m)$ of $\left(\mathfrak{C}^{k-1}(n, m), \check{\mathfrak{g}}_{0}\right)$ represents the Lie algebra of infinitesimal automorphisms of the (local) model $k$-th order differential equation $R_{o}$ of finite type, where

$$
R_{o}=\left\{\frac{\partial^{k} y^{\alpha}}{\partial x_{i_{1}} \cdots \partial x_{i_{k}}}=0 \quad\left(1 \leqq \alpha \leqq m, 1 \leqq i_{1} \leqq \cdots \leqq i_{k} \leqq n\right)\right\} .
$$


The isomorphism $\phi$ of the pseudo-product structure on $R$ preserves the differential system $D=E \oplus F$, which is equivalent to the canonical system $C^{k-1}$ on $J^{k-1}$. Hence, by Bäcklund's Theorem (cf. [Yam83]), $\phi$ is the lift of a point transformation on $J^{0}$ when $m \geqq 2$ and $k \geqq 2$ and is the lift of a contact transformation on $J^{1}$ when $m=1$ and $k \geqq 3$. When $(m, k)=(1,2), \phi$ is the lift of the point transformation on $J^{0}$, since $\phi$ preserves both $D$ and $F=\operatorname{Ker}\left(\pi_{0}^{1}\right)_{*}$. Thus the equivalence of the pseudo-product structure on $R$ is the equivalence of the $k$-th order equation under point or contact transformations. To settle the equivalence problem for the pseudo-projective systems of order $k$ of bidegree $(n, m)$, N.Tanaka constructed the normal Cartan connections of type $\mathfrak{g}^{k}(n, m)$ ([Tan79], [Tan82], [Tan89]).

It is well known that $\mathfrak{g}^{k}(n, m)(k \geqq 2)$ has the following structure ([Tan89],[Yam93], [DKM99], [YY02]);

(1) $k=2 \quad \mathfrak{g}^{2}(n, m)$ is isomorphic to $\mathfrak{s l}(m+n+1, \mathbb{R})$ and has the following gradation:

$$
\mathfrak{s l}(m+n+1, \mathbb{R})=\mathfrak{g}_{-2} \oplus \mathfrak{g}_{-1} \oplus \mathfrak{g}_{0} \oplus \mathfrak{g}_{1} \oplus \mathfrak{g}_{2},
$$

where the gradation is given by subdividing matrices as follows;

$$
\begin{aligned}
\mathfrak{g}_{-2} & =\left\{\left(\begin{array}{lll}
0 & 0 & 0 \\
0 & 0 & 0 \\
\xi & 0 & 0
\end{array}\right) \mid \xi \in W \cong \mathbb{R}^{m}\right\}, \\
\mathfrak{g}_{-1} & =\left\{\left(\begin{array}{lll}
0 & 0 & 0 \\
x & 0 & 0 \\
0 & A & 0
\end{array}\right) \mid x \in V \cong \mathbb{R}^{n}, A \in M(m, n)=W \otimes V^{*}\right\}, \\
\mathfrak{g}_{0} & =\left\{\left(\begin{array}{lll}
a & 0 & 0 \\
0 & B & 0 \\
0 & 0 & C
\end{array}\right) \mid \begin{array}{l}
a \in \mathbb{R}, B \in \mathfrak{g l}(V), C \in \mathfrak{g l}(W), \\
a+\operatorname{tr} B+\operatorname{tr} C=0
\end{array}\right\}, \\
\mathfrak{g}_{1} & =\left\{{ }^{t} X \mid X \in \mathfrak{g}_{-1}\right\}, \quad \mathfrak{g}_{2}=\left\{{ }^{t} X \mid X \in \mathfrak{g}_{-2}\right\},
\end{aligned}
$$

where $V=M(n, 1), W=M(m, 1)$ and $M(a, b)$ denotes the set of $a \times b$ matrices.

(2) $k=3$ and $m=1 \quad \mathfrak{g}^{3}(n, 1)$ is isomorphic to $\mathfrak{s p}(n+1, \mathbb{R})$ and has the following gradation:

$$
\mathfrak{s p}(n+1, \mathbb{R})=\mathfrak{g}_{-3} \oplus \mathfrak{g}_{-2} \oplus \mathfrak{g}_{-1} \oplus \mathfrak{g}_{0} \oplus \mathfrak{g}_{1} \oplus \mathfrak{g}_{2} \oplus \mathfrak{g}_{3}
$$

First we describe

$$
\mathfrak{s p}(n+1, \mathbb{R})=\left\{X \in \mathfrak{g l}(2 n+2, \mathbb{R}) \mid{ }^{t} X J+J X=0\right\},
$$

where

$$
J=\left(\begin{array}{cccc}
0 & 0 & 0 & 1 \\
0 & 0 & I_{n} & 0 \\
0 & -I_{n} & 0 & 0 \\
-1 & 0 & 0 & 0
\end{array}\right) \in \mathfrak{g l}(2 n+2, \mathbb{R}), \quad I_{n}=\left(\delta_{i j}\right) \in \mathfrak{g l}(n, \mathbb{R})
$$


Here $I_{n} \in \mathfrak{g l}(n, \mathbb{R})$ is the unit matrix and the gradation is given again by subdividing matrices as follows;

$$
\begin{aligned}
\mathfrak{g}_{-3} & =\left\{\left(\begin{array}{cccc}
0 & 0 & 0 & 0 \\
0 & 0 & 0 & 0 \\
0 & 0 & 0 & 0 \\
2 a & 0 & 0 & 0
\end{array}\right) \mid \quad a \in \mathbb{R}\right\}, \\
\mathfrak{g}_{-2} & =\left\{\left(\begin{array}{llll}
0 & 0 & 0 & 0 \\
0 & 0 & 0 & 0 \\
\xi & 0 & 0 & 0 \\
0 & { }^{t} \xi & 0 & 0
\end{array}\right) \mid \quad \xi \in \mathbb{R}^{n} \cong V^{*}\right\}, \\
\mathfrak{g}_{-1} & =\left\{\left(\begin{array}{cccc}
0 & 0 & 0 & 0 \\
x & 0 & 0 & 0 \\
0 & A & 0 & 0 \\
0 & 0 & -{ }^{t} x & 0
\end{array}\right) \mid \quad x \in \mathbb{R}^{n}=V, A \in \operatorname{Sym}(n) \cong S^{2}\left(V^{*}\right)\right\}, \\
\mathfrak{g}_{0} & =\left\{\left(\begin{array}{cccc}
b & 0 & 0 & 0 \\
0 & B & 0 & 0 \\
0 & 0 & -{ }^{t} B & 0 \\
0 & 0 & 0 & -b
\end{array}\right) \mid \quad b \in \mathbb{R}, B \in \mathfrak{g l}(V),\right. \\
\mathfrak{g}_{k} & =\left\{{ }^{t} X \mid X \in \mathfrak{g}_{-k}\right\},(k=1,2,3),
\end{aligned}
$$

where $\operatorname{Sym}(n)=\left\{A \in \mathfrak{g l}(n, \mathbb{R}) \mid{ }^{t} A=A\right\}$ is the space of symmetric matrices.

(3) otherwise For vector spaces $V$ and $W$ of dimension $n$ and $m$ respectively, $\mathfrak{g}^{k}(n, m)=\bigoplus_{p \in \mathbb{Z}} \mathfrak{g}_{p}$ has the following description:

$$
\begin{aligned}
\mathfrak{g}_{k} & =\{0\} \quad(k \geqq 2), \quad \mathfrak{g}_{1}=V^{*}, \quad \mathfrak{g}_{0}=\mathfrak{g l}^{\mathfrak{l}}(V) \oplus \mathfrak{g l}(W), \\
\mathfrak{g}_{-1} & =V \oplus W \otimes S^{k-1}\left(V^{*}\right), \quad \mathfrak{g}_{p}=W \otimes S^{k+p}\left(V^{*}\right) \quad(p<-1) .
\end{aligned}
$$

Here the bracket product in $\mathfrak{g}^{k}(n, m)$ is given through the natural tensor operations.

For the structure of $\mathfrak{g}^{k}(n, m)$ in case $(3)$, we observe the following points. We put

$$
\begin{gathered}
\mathfrak{l}=V \oplus \mathfrak{g}_{0} \oplus \mathfrak{g}_{1}=\left(V \oplus \mathfrak{g l}(V) \oplus V^{*}\right) \oplus \mathfrak{g l}(W) \\
\cong \mathfrak{s l}(\hat{V}) \oplus \mathfrak{g l}(W), \\
S=W \otimes S^{k-1}\left(\hat{V}^{*}\right), \quad \hat{V}=\mathbb{R} \oplus V .
\end{gathered}
$$

where the gradation of the first kind; $\mathfrak{s l}(\hat{V})=V \oplus \mathfrak{g l}(V) \oplus V^{*}$ is given by subdividing matrices corresponding to the decomposition $\hat{V}=\mathbb{R} \oplus V$.

Then

$$
S^{k-1}\left(\hat{V}^{*}\right) \cong \bigoplus_{\ell=0}^{k-1} S^{\ell}\left(V^{*}\right),
$$

and $S$ is a faithful irreducible $\mathfrak{l}$-module such that $\mathfrak{l}=\mathfrak{l}_{-1} \oplus \mathfrak{l}_{0} \oplus \mathfrak{l}_{1}$ is a reductive graded Lie algebras, where $\mathfrak{l}_{-1}=V, \mathfrak{l}_{0}=\mathfrak{g}_{0}, \mathfrak{l}_{1}=\mathfrak{g}_{1}$. Moreover $\mathfrak{g}^{k}(n, m) \cong S \oplus \mathfrak{l}$ is the semi-direct product of $\mathfrak{l}$ by $S$.

In the following sections, we will seek to find other parabolic geometries associated with differential equations of finite type, which are the generalizations of the above cases (1) and (2). 
2.2. Pseudo-product GLA of type $(\mathfrak{l}, S)$. We will now give the notion of the pseudoproduct GLA of type $(\mathfrak{l}, S)$, generalizing the pseudo-projective GLA of order $k$ of bidegree $(n, m)$.

Let $\mathfrak{g}=\bigoplus_{p \in \mathbb{Z}} \mathfrak{g}_{p}$ be a (transitive) graded Lie algebra (GLA) over the field $\mathbb{K}$ such that the negative part $\mathfrak{m}=\bigoplus_{p<0} \mathfrak{g}_{p}$ is a FGLA, i.e., $\left[\mathfrak{g}_{p}, \mathfrak{g}_{-1}\right]=\mathfrak{g}_{p-1}$ for $p<0$, where $\mathbb{K}$ is the field $\mathbb{R}$ of real numbers or the field $\mathbb{C}$ of complex numbers. Let $\mathfrak{e}$ and $\mathfrak{f}$ be subspaces of $\mathfrak{g}_{-1}$. Then the system $\mathfrak{G}=\left(\mathfrak{g},\left(\mathfrak{g}_{p}\right)_{p \in \mathbb{Z}}, \mathfrak{e}, \mathfrak{f}\right)$ is called a pseudo-product GLA (PPGLA) of irreducible type if the following conditions hold:

(1) $\mathfrak{g}$ is transitive, i.e., for each $k \geqq 0$, if $X \in \mathfrak{g}_{k}$ and $\left[X, \mathfrak{g}_{-1}\right]=0$, then $X=0$.

(2) $\mathfrak{g}_{-1}=\mathfrak{e} \oplus \mathfrak{f}, \quad[\mathfrak{e}, \mathfrak{e}]=[\mathfrak{f}, \mathfrak{f}]=0$.

(3) $\left[\mathfrak{g}_{0}, \mathfrak{e}\right] \subset \mathfrak{e}$ and $\left[\mathfrak{g}_{0}, \mathfrak{f}\right] \subset \mathfrak{f}$.

(4) $\mathfrak{g}_{-2} \neq 0$ and the $\mathfrak{g}_{0}$-modules $\mathfrak{e}$ and $\mathfrak{f}$ are irreducible.

It is known that $\mathfrak{g}$ becomes finite dimensional under these conditions (see [Tan85], [Yat88]).

As a typical example, starting from a reductive GLA $\mathfrak{l}=\mathfrak{l}_{-1} \oplus \mathfrak{l}_{0} \oplus \mathfrak{l}_{1}$ and a faithful irreducible l-module $S$, we define the pseudo-product GLA $\mathfrak{g}=\bigoplus_{p \in \mathbb{Z}} \mathfrak{g}_{p}$ of type $(\mathfrak{l}, S)$ as follows: Let $\mathfrak{l}=\mathfrak{l}_{-1} \oplus \mathfrak{l}_{0} \oplus \mathfrak{l}_{1}$ be a finite dimensional reductive GLA of the first kind such that

(1) The ideal $\hat{\mathfrak{l}}=\mathfrak{l}_{-1} \oplus\left[\mathfrak{l}_{-1}, \mathfrak{l}_{1}\right] \oplus \mathfrak{l}_{1}$ of $\mathfrak{l}$ is a simple Lie algebra.

(2) The center $\mathfrak{z}(\mathfrak{l})$ of $\mathfrak{l}$ is contained in $\mathfrak{l}_{0}$.

Let $S$ be a finite dimensional faithful irreducible $\mathfrak{l}$-module. We put

$$
S_{-1}=\left\{s \in S \mid \mathfrak{l}_{1} \cdot s=0\right\}
$$

and

$$
S_{p}=\operatorname{ad}\left(\mathfrak{l}_{-1}\right)^{-p-1} S_{-1} \text { for } p<0
$$

We form the semi-direct product $\mathfrak{g}$ of $\mathfrak{l}$ by $S$, and put

$$
\begin{gathered}
\mathfrak{g}=S \oplus \mathfrak{l}, \quad[S, S]=0 \\
\mathfrak{g}_{k}=\mathfrak{l}_{k}(k \geqq 0), \quad \mathfrak{g}_{-1}=\mathfrak{l}_{-1} \oplus S_{-1}, \\
\mathfrak{g}_{p}=S_{p}(p<-1) .
\end{gathered}
$$

Namely $\mathfrak{g}$ is a subalgebra of the Lie algebra $\mathfrak{A}(S)=S \oplus \mathfrak{g l}(S)$ of infinitesimal affine transformations of $S$.

Then we have (Lemma 2.1 [YY02])

Lemma 2.1. Notations being as above,

(1) $S=\bigoplus_{p=-1}^{-\mu} S_{p}$, where $S_{-\mu}=\left\{s \in S \mid\left[\mathfrak{l}_{-1}, s\right]=0\right\}$.

(2) $\mathfrak{m}=\bigoplus_{p<0} \mathfrak{g}_{p}$ is generated by $\mathfrak{g}_{-1}$.

(3) $\left[S_{p}, \mathfrak{l}_{1}\right]=S_{p+1}$ for $p<-1$.

(4) $S_{p}$ is naturally embedded as a subspace of $W \otimes S^{\mu+p}\left(\mathfrak{l}_{-1}{ }^{*}\right)$ through the bracket operation in $\mathfrak{m}$, where $W=S_{-\mu}$.

(5) $S_{-1}, S_{-\mu}$ are irreducible $\mathfrak{l}_{0}$-modules.

Thus $\mathfrak{m}$ is a graded subalgebra of $\mathfrak{C}^{\mu-1}(V, W)$, which has the splitting $\mathfrak{g}_{-1}=\mathfrak{l}_{-1} \oplus S_{-1}$, where $V=\mathfrak{l}_{-1}$ and $W=S_{-\mu}$. Hence $\mathfrak{m}$ is a symbol algebra of $\mu$-th order differential equations of finite type, which is called the typical symbol of type $(\mathfrak{l}, S)$. Moreover the system $\mathfrak{G}=\left(\mathfrak{g},\left(\mathfrak{g}_{p}\right)_{p \in \mathbb{Z}}, \mathfrak{l}_{-1}, S_{-1}\right)$ becomes a PPGLA of irreducible type, which is called the pseudo-product GLA of type $(\mathfrak{l}, S)$.

This class of higher order (linear) differential equations of finite type were first appeared in the work of Y.Se-ashi [Sea88]. 
2.3. Prolongation Theorem. Let $\mathfrak{G}=\left(\mathfrak{g},\left(\mathfrak{g}_{p}\right)_{p \in \mathbb{Z}}, \mathfrak{l}_{-1}, S_{-1}\right)$ be a pseudo-product GLA of type $(\mathfrak{l}, S)$, i.e., $\mathfrak{g}=S \oplus \mathfrak{l}$ is endowed with the gradation $\left(\mathfrak{g}_{p}\right)_{p \in \mathbb{Z}}, \mathfrak{g}=\bigoplus_{p=-\mu}^{1} \mathfrak{g}_{p}$ given in $\S 2.2$. $\mathfrak{g}$ has also another gradation $\left(\mathfrak{b}_{p}\right)_{p \in \mathbb{Z}}, \mathfrak{g}=\bigoplus_{p=-1}^{0} \mathfrak{b}_{p}$, given by $\mathfrak{b}_{-1}=S$ and $\mathfrak{b}_{0}=\mathfrak{l}$. Thus $\mathfrak{g}$ has a bigradation $\left(\mathfrak{g}_{p, q}\right)_{p, q \in \mathbb{Z}}$, where $\mathfrak{g}_{p, q}=\mathfrak{g}_{p} \cap \mathfrak{b}_{q}$. We have the cohomology group $H^{*}(\mathfrak{G})=H^{*}(\mathfrak{m}, \mathfrak{g})$ associated with the adjoint representation of $\mathfrak{m}=\mathfrak{g}_{-}$on $\mathfrak{g}$, that is, the cohomology space of the cochain complex $C^{*}(\mathfrak{G})=\bigoplus C^{p}(\mathfrak{G})$ with the coboundary operator $\partial: C^{p}(\mathfrak{G}) \longrightarrow C^{p+1}(\mathfrak{G})$, where $C^{p}(\mathfrak{G})=\operatorname{Hom}\left(\bigwedge^{p} \mathfrak{g}_{-}, \mathfrak{g}\right)$. We put

$$
\begin{aligned}
& C^{p}(\mathfrak{G})_{r, s}=\left\{\omega \in C^{p}(\mathfrak{G}) \mid\right. \\
& \omega\left(\mathfrak{g}_{i_{1}, j_{1}} \wedge \cdots \wedge \mathfrak{g}_{i_{p}, j_{p}}\right) \subset \mathfrak{g}_{i_{1}+\cdots+i_{p}+r, j_{1}+\cdots+j_{p}+s} \\
&\left.\quad \text { for all } i_{1}, \ldots, i_{p}, j_{1}, \ldots, j_{p}\right\} .
\end{aligned}
$$

As is easily seen, $C^{*}(\mathfrak{G})_{r, s}=\bigoplus_{p} C^{p}(\mathfrak{G})_{r, s}$ is a subcomplex of $C^{*}(\mathfrak{G})$. Denoting its cohomology space by $H(\mathfrak{G})_{r, s}=\bigoplus H^{p}(\mathfrak{G})_{r, s}$, we obtain the direct sum decomposition

$$
H^{*}(\mathfrak{G})=\bigoplus_{p, r, s} H^{p}(\mathfrak{G})_{r, s}
$$

The cohomology space, endowed with this tri-gradation, is called the generalized Spencer cohomology space of the PPGLA $\mathfrak{G}$ of type $(\mathfrak{l}, S)$. Note that $H^{1}(\mathfrak{G})_{0,0}=0$ if and only if $\mathfrak{g}_{0}$ coincides with the Lie algebra of derivations of $\mathfrak{m}$ such that $D\left(\mathfrak{g}_{p}\right) \subset \mathfrak{g}_{p}(p<0)$, $D\left(\mathfrak{l}_{-1}\right) \subset \mathfrak{l}_{-1}$ and $D\left(S_{-1}\right) \subset S_{-1}$.

From now on, we assume that the ground field is the field $\mathbb{C}$ of complex numbers for the sake of simplicity. For the discussion over $\mathbb{R}$, the corresponding results will be obtained easily through the argument of complexification as in $§ 3.2$ in [Yam93]. We set $\hat{\mathfrak{l}}=\mathfrak{l}_{-1} \oplus\left[\mathfrak{l}_{-1}, \mathfrak{l}_{1}\right] \oplus \mathfrak{l}_{1}$ and $\mathfrak{u}=\mathcal{D}\left(\mathfrak{z}_{\mathfrak{l}}(\hat{\mathfrak{l}})\right)$; then $\mathfrak{l}=\hat{\mathfrak{l}} \oplus \mathfrak{u} \oplus \mathfrak{z}(\mathfrak{l}), \mathcal{D}(\mathfrak{l})=\hat{\mathfrak{l}} \oplus \mathfrak{u}$ and $\hat{\mathfrak{l}}=\mathfrak{l}_{-1} \oplus \hat{\mathfrak{l}}_{0} \oplus \mathfrak{l}_{1}$, where $\hat{\mathfrak{l}}_{0}=\left[\mathfrak{l}_{-1}, \mathfrak{l}_{1}\right]$, is a simple GLA. Let us take a Cartan subalgebra $\mathfrak{h}$ of $\mathfrak{l}$ such that $\mathfrak{h} \subset \mathfrak{l}_{0}$. Then $\mathfrak{h} \cap \hat{\mathfrak{l}}($ resp. $\mathfrak{h} \cap \mathfrak{u})$ is a Cartan subalgebra of $\hat{\mathfrak{l}}($ resp. $\mathfrak{u})$. Let $\Delta=\left\{\alpha_{1}, \ldots, \alpha_{\ell}\right\}$ (resp. $\left.\Delta^{\prime}=\left\{\beta_{1}, \ldots, \beta_{m}\right\}\right)$ be a simple root system of $(\hat{\mathfrak{l}}, \mathfrak{h} \cap \hat{\mathfrak{l}})($ resp. $(\mathfrak{u}, \mathfrak{h} \cap \mathfrak{u}))$ such that $\alpha(Z) \geqq 0$ for all $\alpha \in \Delta$, where $Z$ is the characteristic element of the GLA $\mathfrak{l}=\mathfrak{l}_{-1} \oplus \mathfrak{l}_{0} \oplus \mathfrak{l}_{1}$. We assume that $\hat{\mathfrak{l}}$ is a simple Lie algebra of type $X_{\ell}$. We set $\Delta_{1}=\{\alpha \in \Delta \mid \alpha(Z)=1\}$. It is well known that the pair $\left(X_{\ell}, \Delta_{1}\right)$ is one of the following type (up to a diagram automorphism) (cf. $\S 3$ in [Yam93]):

$$
\begin{aligned}
& \left(A_{\ell},\left\{\alpha_{i}\right\}\right)(1 \leqq i \leqq[(\ell+1) / 2]),\left(B_{\ell},\left\{\alpha_{1}\right\}\right)(\ell \geqq 3),\left(C_{\ell},\left\{\alpha_{\ell}\right\}\right)(\ell \geqq 2), \\
& \left(D_{\ell},\left\{\alpha_{1}\right\}\right)(\ell \geqq 4),\left(D_{\ell},\left\{\alpha_{\ell-1}\right\}\right)(\ell \geqq 5),\left(E_{6},\left\{\alpha_{1}\right\}\right), \quad\left(E_{7},\left\{\alpha_{7}\right\}\right) .
\end{aligned}
$$

We denote by $\left\{\varpi_{1}, \ldots, \varpi_{\ell}\right\}$ (resp. $\left\{\pi_{1}, \ldots, \pi_{n}\right\}$ ) the set of fundamental weights relative to $\Delta$ (resp. $\Delta^{\prime}$ ). Since $S$ is a faithful $\mathfrak{l}$-module, we have $\operatorname{dim} \mathfrak{z}(\mathfrak{l}) \leqq 1$. Assume that $\mathfrak{z}(\mathfrak{l}) \neq\{0\}$. Let $\sigma$ be the element of $\mathfrak{z}(\mathfrak{l})^{*}$ such that $\sigma(J)=1$, where $J$ is the characteristic element of the GLA $\mathfrak{g}=\mathfrak{b}_{-1} \oplus \mathfrak{b}_{0}$. Namely $J=-i d_{S} \in \mathfrak{z}(\mathfrak{l}) \subset \mathfrak{b}_{0}=\mathfrak{l}$ as the element of $\mathfrak{g l}(S)$. There is an irreducible $\hat{\mathfrak{l}}$-module $T$ (resp. $\mathfrak{z} \mathfrak{l}(\hat{\mathfrak{l}})$-module $U$ ) with highest weight $\chi$ (resp. $\eta-\sigma$ ) such that $S=\mathfrak{b}_{-1}$ is isomorphic to $U \otimes T$ as an l-module, where $\eta$ is a weight of $\mathfrak{u}$. Then we have (Lemma 4.5 [YY02])

Lemma 2.2. $H^{1}(\mathfrak{G})_{0,0}=0$ if and only if $\mathfrak{z}(\hat{\mathfrak{l}})$ is isomorphic to $\mathfrak{g l}(U)$ and $\eta=\pi_{1}$. Especially, when $\mathcal{D}(\mathfrak{l})=\hat{\mathfrak{l}}, H^{1}(\mathfrak{G})_{0,0}=0$ if and only if $\mathfrak{l}=\hat{\mathfrak{l}} \oplus \mathfrak{z}(\mathfrak{l})$, where $\mathfrak{z}(\mathfrak{l})=\langle J\rangle$.

Thus, when $H^{1}(\mathfrak{G})_{0,0}=0$, the semisimple GLA $\mathcal{D}(\mathfrak{l})$ is of type $\left(X_{\ell} \times A_{n},\left\{\alpha_{i}\right\}\right)$ and $S$ is an irreducible $\mathcal{D}(\mathfrak{l})$-module with highest weight $\Xi=\chi+\pi_{1}$ when $\operatorname{dim} U>1$ and $\mathcal{D}(\mathfrak{l})$ is 
of type $\left(X_{\ell},\left\{\alpha_{i}\right\}\right)$ and $S$ is an irreducible $\hat{\mathfrak{l}}$-module with highest weight $\chi$, when $\mathcal{D}(\mathfrak{l})=\hat{\mathfrak{l}}$ (i.e., when $\operatorname{dim} U=1$ ).

The following theorem was obtained in Theorem 5.4 [YY02] as the answer to the following question:

$$
\text { When is } \mathfrak{g} \text { the prolongation of } \mathfrak{m} \text { or }\left(\mathfrak{m}, \mathfrak{g}_{0}\right) \text { ? }
$$

In the following theorem $(a)$, the simple graded Lie algebra $\mathfrak{b}=\check{\mathfrak{g}}=\bigoplus_{p \in \mathbb{Z}} \check{\mathfrak{g}}_{p}$ is described by $\left(Y_{\ell+n+1}, \Sigma_{1}\right)$ such that $\mathfrak{g}=\bigoplus_{p=-\mu}^{1} \mathfrak{g}_{p}$ is a graded subalgebra of $\check{\mathfrak{g}}=\bigoplus_{p=-\mu}^{\mu} \check{\mathfrak{g}}_{p}$ satisfying $\mathfrak{g}_{p}=\check{\mathfrak{g}}_{p}$ for $p \leqq 0$.

Theorem 2.1. Let $\mathfrak{G}$ be a pseudo-product $G L A$ of type $(\mathfrak{l}, S)$ satisfying the condition $H^{1}(\mathfrak{G})_{0,0}=0$. Let $\mathfrak{b}=\bigoplus_{p \in \mathbb{Z}} \mathfrak{b}_{p}$ be the prolongation of $\mathfrak{g}=\mathfrak{b}_{-1} \oplus \mathfrak{b}_{0}$, where $\mathfrak{b}_{-1}=S$ and $\mathfrak{b}_{0}=\mathfrak{l}$. Then $\mathfrak{g}=\bigoplus_{p \in \mathbb{Z}} \mathfrak{g}_{p}$ is the prolongation of $\mathfrak{m}=\oplus_{p<0} \mathfrak{g}_{p}$ except for the following three cases.

(a) $\operatorname{dim} \mathfrak{b}<\infty$ and $\mathfrak{b}_{1} \neq 0 \quad\left(\mathfrak{b}=\mathfrak{b}_{-1} \oplus \mathfrak{b}_{0} \oplus \mathfrak{b}_{1}\right.$ : simple $)$

\begin{tabular}{|c|c|c|c|c|}
\hline $\mathcal{D}(\mathfrak{l})=[\mathfrak{l}, \mathfrak{l}]$ & $\Delta_{1}$ & $\mathfrak{b}_{-1}=S$ & $\mathfrak{\mathfrak { g }}=Y_{\ell+n+1}$ & $\Sigma_{1}$ \\
\hline$A_{\ell} \times A_{n}$ & $\left\{\alpha_{i}\right\}$ & $\varpi_{\ell}+\pi_{1}$ & $A_{\ell+n+1}$ & $\left\{\gamma_{i}, \gamma_{\ell+1}\right\}$ \\
\hline$A_{\ell}$ & $\left\{\alpha_{i}\right\}$ & $2 \varpi_{l}$ & $C_{\ell+1}$ & $\left\{\gamma_{i}, \gamma_{\ell+1}\right\}$ \\
\hline$A_{\ell}(\ell \geqq 3)$ & $\left\{\alpha_{i}\right\}$ & $\varpi_{\ell-1}$ & $D_{\ell+1}$ & $\left\{\gamma_{i}, \gamma_{\ell+1}\right\}$ \\
\hline$B_{\ell}(\ell \geqq 2)$ & $\left\{\alpha_{1}\right\}$ & $\varpi_{1}$ & $B_{\ell+1}$ & $\left\{\gamma_{1}, \gamma_{2}\right\}$ \\
\hline$D_{\ell}(\ell \geqq 4)$ & $\left\{\alpha_{1}\right\}$ & $\varpi_{1}$ & $D_{\ell+1}$ & $\left\{\gamma_{1}, \gamma_{2}\right\}$ \\
\hline$D_{\ell}(\ell \geqq 4)$ & $\left\{\alpha_{\ell}\right\}$ & $\varpi_{1}$ & $D_{\ell+1}$ & $\left\{\gamma_{1}, \gamma_{\ell+1}\right\}$ \\
\hline$D_{5}$ & $\left\{\alpha_{1}\right\}$ & $\varpi_{5}$ & $E_{6}$ & $\left\{\gamma_{1}, \gamma_{6}\right\}$ \\
\hline$D_{5}$ & $\left\{\alpha_{5}\right\}$ & $\varpi_{5}$ & $E_{6}$ & $\left\{\gamma_{1}, \gamma_{3}\right\}$ \\
\hline$D_{5}$ & $\left\{\alpha_{4}\right\}$ & $\varpi_{5}$ & $E_{6}$ & $\left\{\gamma_{1}, \gamma_{2}\right\}$ \\
\hline$E_{6}$ & $\left\{\alpha_{6}\right\}$ & $\varpi_{6}$ & $E_{7}$ & $\left\{\gamma_{6}, \gamma_{7}\right\}$ \\
\hline$E_{6}$ & $\left\{\alpha_{1}\right\}$ & $\varpi_{6}$ & $E_{7}$ & $\left\{\gamma_{1}, \gamma_{7}\right\}$ \\
\hline
\end{tabular}

In this case $\left(Y_{\ell+n+1}, \Sigma_{1}\right)$ is the prolongation of $\mathfrak{m}$ except for $\left(A_{\ell+n+1},\left\{\gamma_{1}, \gamma_{\ell+1}\right\}\right)$ and $\left(C_{\ell+1},\left\{\gamma_{1}, \gamma_{\ell+1}\right\}\right)$. Moreover the latter two are the prolongations of $\left(\mathfrak{m}, \mathfrak{g}_{0}\right)$.

(b) $\operatorname{dim} \mathfrak{b}=\infty$

\begin{tabular}{|c|c|c|c|}
\hline $\mathcal{D}(\mathfrak{l})$ & $\Delta_{1}$ & $\mathfrak{b}_{-1}$ & $\mathfrak{g}\left(\mathfrak{m}, \mathfrak{g}_{0}\right)$ \\
\hline$A_{\ell}$ & $\left\{\alpha_{i}\right\}$ & $\varpi_{\ell}$ & $\left(A_{\ell+1},\left\{\gamma_{i}, \gamma_{\ell+1}\right\}\right)$ \\
\hline$C_{\ell}$ & $\left\{\alpha_{\ell}\right\}$ & $\varpi_{1}$ & $\mathfrak{g}$ \\
\hline
\end{tabular}

In $\left(C_{\ell},\left\{\alpha_{\ell}\right\}\right)$-case, $\mu=2$

$$
\begin{array}{rlrl}
S_{-2} & =V^{*}, \quad S_{-1}=V, & \mathfrak{l}_{-1}=S^{2}\left(V^{*}\right), \\
\mathfrak{l}_{0}=V \otimes V^{*} \oplus \mathbb{C}, & \mathfrak{l}_{1}=S^{2}(V)
\end{array}
$$


(c) $\mathfrak{g}$ is a pseudo-projective GLA, i.e., $\mathcal{D}(\mathfrak{l})=\left(A_{\ell} \times A_{n},\left\{\alpha_{1}\right\}\right), \Xi=k \varpi_{\ell}+\pi_{1},(k \geqq$ $2, n \geqq 1)$, or $\mathcal{D}(\mathfrak{l})=\left(A_{\ell},\left\{\alpha_{1}\right\}\right), \chi=k \varpi_{\ell},(k \geqq 3, n=0)$

$$
\begin{gathered}
S_{-\mu}=W, \quad S_{p}=W \otimes S^{\mu+p}\left(V^{*}\right)(-\mu<p<0), \\
\mathfrak{l}_{-1}=V, \quad \mathfrak{l}_{0}=\mathfrak{g l}(V) \oplus \mathfrak{g l}(W), \quad \mathfrak{l}_{1}=V^{*},
\end{gathered}
$$

where $\mu=k+1, \operatorname{dim} V=\ell$ and $\operatorname{dim} W=n+1$.

In this case $\mathfrak{g}$ is the prolongation of $\left(\mathfrak{m}, \mathfrak{g}_{0}\right)$.

By Proposition 4.4.1 in [Sea88], the Lie algebra of infinitesimal linear automorphisms of the model equation of type $(\mathfrak{l}, S)$ coincides with $\mathfrak{l}$. Hence the cases $(a)$ and $(b)$ of the above theorem exhaust classes of the equations of type $(\mathfrak{l}, S)$, for which the model equations admit non trivial nonlinear automorphisms. These cases correspond to the parabolic geometries associated with differential equations of finite type, which generalize the case of second and third order ODEs, mentioned in the introduction. More precisely, in the cases of $\left(A_{\ell+1},\left\{\gamma_{1}, \gamma_{i}\right\}\right)$ and $\left(C_{\ell+1},\left\{\gamma_{1}, \gamma_{\ell+1}\right\}\right), \mathfrak{m}$ coincides with the symbol algebra of the canonical system of the first or second order jet spaces (cf. §4.5 [Yam93]) and $\mathfrak{g}_{0}$ determines the splitting of $\mathfrak{g}_{-1}$, hence the parabolic geometries associated with these graded Lie algebras are geometries of the pseudo-product structures on the first or second order jet spaces. In fact the parabolic geometry associated with $\left(A_{m+n},\left\{\gamma_{1}, \gamma_{n+1}\right\}\right)$ is the geometry of the pseudo-projective system of order 2 of bidegree $(n, m)$ and the parabolic geometry associated with $\left(C_{n+1},\left\{\gamma_{1}, \gamma_{n+1}\right\}\right)$ is the geometry of the pseudo-projective system of order 3 of bidegree $(n, 1)$ (see the following section).

In the other cases of the above theorem $(a),\left(Y_{\ell+n+1}, \Sigma_{1}\right)$ is the prolongation of $\mathfrak{m}$. This fact implies that the parabolic geometries associated with these graded Lie algebras are geometries of regular differential system of type $\mathfrak{m}$, which have the (almost) pseudo-product structure corresponding to $\mathfrak{g}_{-1}=S_{-1} \oplus \mathfrak{l}_{-1}$. Moreover every isomorphism of these regular differential system preserves this pseudo-product structure. Thus the parabolic geometries associated with $\left(Y_{\ell+n+1}, \Sigma_{1}\right)$ have the canonical (almost) pseudo-product structures in the regular differential system of type $\mathfrak{m}$ corresponding to the splitting $\mathfrak{g}_{-1}=S_{-1} \oplus \mathfrak{l}_{-1}$

In the following sections, we will calculate the explicit forms of the typical symbols of type $(\mathfrak{l}, S)$ of the above cases and describe the above (almost) pseudo-product structures as differential equations of finite type.

\section{Symbol of the ClASSICAL CASES}

In this section we will describe the symbol algebra $\mathfrak{m}=\bigoplus_{p<0} \mathfrak{g}_{p}$ explicitly as the subalgebra of $\mathfrak{C}^{\mu-1}(V, W)$, where $V=\mathfrak{l}_{-1}$ and $W=S_{-\mu}$, by utilizing the explicit matrices description of the graded Lie algebra $\check{\mathfrak{g}}=\bigoplus_{p \in \mathbb{Z}} \check{\mathfrak{g}}_{p}$ of type $\left(Y_{L}, \Sigma_{1}\right)$. For an explicit matrices description of the graded Lie algebra $\left(Y_{L}, \Sigma_{1}\right)$, we refer the reader to $\S 4.4$ in [Yam93]. By this calculation, we can explicitly write down the class of differential equations of finite type corresponding to the pseudo-product structure associated with the simple graded Lie algebra $\left(Y_{L}, \Sigma_{1}\right)$. In this section, we shall discuss in the complex analytic or the real $C^{\infty}$ category depending on whether $\mathbb{K}=\mathbb{C}$ or $\mathbb{R}$.

Case (1) $\left[\left(A_{\ell} \times A_{n},\left\{\alpha_{i}\right\}\right), \varpi_{\ell}+\pi_{1},\left(A_{\ell+n+1},\left\{\gamma_{i}, \gamma_{\ell+1}\right\}\right)\right](1<i \leqq \ell, n \geqq 0, \ell \geqq 2)$. This includes the first case of $(b)$ in the above theorem as the case $n=0$. 
$\mathfrak{b}=\mathfrak{b}_{-1} \oplus \mathfrak{b}_{0} \oplus \mathfrak{b}_{1}$ is described by $\left(A_{\ell+n+1},\left\{\gamma_{\ell+1}\right\}\right)$ and $\check{\mathfrak{g}}=\bigoplus_{p=-\mu}^{\mu} \check{\mathfrak{g}}_{p}$ is described by $\left(A_{\ell+n+1},\left\{\gamma_{i}, \gamma_{\ell+1}\right\}\right)$. Hence $\mu=2$ and we obtain the following matrix representation of $\check{\mathfrak{g}}=\mathfrak{b}=\mathfrak{s l}(\ell+n+2, \mathbb{K})$ :

$$
\mathfrak{s l}(\ell+n+2, \mathbb{K})=\mathfrak{g}_{-2} \oplus \mathfrak{g}_{-1} \oplus \mathfrak{g}_{0} \oplus \check{\mathfrak{g}}_{1} \oplus \check{\mathfrak{g}}_{2}=S \oplus \mathfrak{l} \oplus S^{*},
$$

where the gradation is given by subdividing matrices as follows;

$$
\begin{aligned}
& \mathfrak{g}_{-2}=S_{-2}=\left\{\left(\begin{array}{lll}
0 & 0 & 0 \\
0 & 0 & 0 \\
A & 0 & 0
\end{array}\right) \mid A \in M(n+1, i) \cong U \otimes T_{0}^{*}\right\}, \\
& \mathfrak{g}_{-1}=S_{-1} \oplus \mathfrak{l}_{-1}, \\
& S_{-1}=U \otimes T_{-1}^{*}=\left\{\left(\begin{array}{lll}
0 & 0 & 0 \\
0 & 0 & 0 \\
0 & B & 0
\end{array}\right) \mid B \in M(n+1, j)\right\}, \\
& \mathfrak{l}_{-1}=T_{-1} \otimes T_{0}^{*}=\left\{\left(\begin{array}{ccc}
0 & 0 & 0 \\
C & 0 & 0 \\
0 & 0 & 0
\end{array}\right) \mid C \in M(j, i)\right\}, \\
& \mathfrak{g}_{0}=\check{\mathfrak{l}}_{0} \oplus \mathfrak{u}=\left\{\left(\begin{array}{ccc}
F & 0 & 0 \\
0 & G & 0 \\
0 & 0 & H
\end{array}\right) \mid F \in \mathfrak{g l}\left(T_{0}\right), G \in \mathfrak{g l}\left(T_{-1}\right), H \in \mathfrak{g l}(U),\right. \\
& \check{\mathfrak{g}}_{1}= \begin{cases}{ }^{t} X \mid X \in \operatorname{tr} G+\operatorname{tr} H=0 \\
\left.\mathfrak{g}_{-1}\right\}, & \check{\mathfrak{g}}_{2}=\left\{{ }^{t} X \mid X \in \mathfrak{g}_{-2}\right\},\end{cases}
\end{aligned}
$$

where $i+j=\ell+1, U=\mathbb{K}^{n+1}, T=T_{0} \oplus T_{-1}=\mathbb{K}^{\ell+1}, T_{0}=\mathbb{K}^{i}, T_{-1}=\mathbb{K}^{j}$ and $M(a, b)$ denotes the set of $a \times b$ matrices. Thus we have

$$
S=U \otimes T^{*}, \quad \mathfrak{l}=\mathfrak{s l}(T) \oplus \mathfrak{g l}(U), \quad \text { and } \quad \check{\mathfrak{g}}=\mathfrak{s l}(T \oplus U) .
$$

We will divide the argument into the following two cases. We first consider the typical case:

(i) $i=\ell \geqq 2, n=0$.

We have $j=1$ and $n=0$ in the above matrix description. Hence $\operatorname{dim} \mathfrak{l}_{-1}=\operatorname{dim} S_{-2}=\ell$ and $\operatorname{dim} S_{-1}=1$. We put $\mathfrak{l}_{-1}=S_{-2}=V$. Then

$$
\mathfrak{m}=S_{-2} \oplus\left(S_{-1} \oplus \mathfrak{l}_{-1}\right)=\left\{\left(\begin{array}{lll}
0 & 0 & 0 \\
x & 0 & 0 \\
y & a & 0
\end{array}\right)=\check{y}+\hat{a}+\hat{x} \mid x, y \in V=M(1, \ell), a \in \mathbb{K}\right\} .
$$

By a direct calculation, we have $[\hat{a}, \hat{x}]=(\check{a x}) \in S_{-2}=V$, i.e., $y=a x$. Thus $S_{-1}$ is embedded as the 1-dimensional subspace of scalar multiplications of $V \otimes V^{*}=S_{-2} \otimes\left(\mathfrak{l}_{-1}\right)^{*}$ through the bracket operation in $\mathfrak{m}$. This implies that the model equation of our typical symbol $\mathfrak{m}=\mathfrak{g}_{-2} \oplus \mathfrak{g}_{-1} \subset \mathfrak{C}^{1}(V, V)$ is given by

$$
\frac{\partial y_{p}}{\partial x_{q}}=\delta_{p q} \frac{\partial y_{1}}{\partial x_{1}} \quad \text { for } \quad 1 \leqq p, q \leqq \ell .
$$

where $y_{1}, \ldots, y_{\ell}$ are dependent variables and $x_{1}, \ldots, x_{\ell}$ are independent variables. By a direct calculation, we see that the prolongation of the first order system (3.1) is given by

$$
\frac{\partial^{2} y_{p}}{\partial x_{q} \partial x_{r}}=0 \quad \text { for } \quad 1 \leqq p, q, r \leqq \ell
$$


(ii) otherwise.

We have $S_{-2}=U \otimes T_{0}^{*} \cong M(n+1, i), S_{-1}=U \otimes T_{-1}^{*} \cong M(n+1, j)$ and $\mathfrak{l}_{-1}=$ $T_{-1} \otimes T_{0}^{*} \cong M(j, i)$. Then

$$
\begin{aligned}
\mathfrak{m} & =S_{-2} \oplus\left(S_{-1} \oplus \mathfrak{l}_{-1}\right) \\
& =\left\{\left(\begin{array}{lll}
0 & 0 & 0 \\
X & 0 & 0 \\
Y & A & 0
\end{array}\right)=\check{Y}+\hat{A}+\hat{X} \mid Y \in M(n+1, i), A \in M(n+1, j), X \in M(j, i)\right\} .
\end{aligned}
$$

By a direct calculation, we have $[\hat{A}, \hat{X}]=(A X)^{\sim} \in S_{-2}$, i.e., $y^{\alpha}=\sum_{\tau=1}^{j} a_{\tau}^{\alpha} x^{\tau}$, where $y^{\alpha}$ is the $\alpha$-th row of $Y, x^{\tau}$ is the $\tau$-th row of $X$ and $A=\left(a_{\tau}^{\alpha}\right)$. From $(i)$, we see that the model equation of our typical symbol $\mathfrak{m}=\mathfrak{g}_{-2} \oplus \mathfrak{g}_{-1} \subset \mathfrak{C}^{1}\left(\mathfrak{l}_{-1}, S_{-2}\right)$ is given by

$$
\frac{\partial y_{p}^{\alpha}}{\partial x_{q}^{\tau}}=\delta_{p q} \frac{\partial y_{1}^{\alpha}}{\partial x_{1}^{\tau}} \quad \text { for } \quad \alpha=1, \ldots, n+1, \quad \tau=1, \ldots, j, \quad 1 \leqq p, q \leqq i,
$$

where $y_{1}^{1}, \ldots, y_{i}^{1}, \ldots, y_{1}^{n+1}, \ldots, y_{i}^{n+1}$ are dependent variables and $x_{1}^{1}, \ldots, x_{i}^{1}, \ldots, x_{1}^{j}, \ldots, x_{i}^{j}$ are independent variables. By a direct calculation, we see that the prolongation of the first order system (3.3) is given by

$$
\frac{\partial^{2} y_{p}^{\alpha}}{\partial x_{q}^{\tau} \partial x_{r}^{v}}=0 \quad \text { for } \quad \alpha=1, \ldots, n+1, \quad 1 \leqq \tau, v \leqq j, \quad 1 \leqq p, q, r \leqq i
$$

Case $(2)\left[\left(A_{\ell} \times A_{n},\left\{\alpha_{1}\right\}\right), \varpi_{\ell}+\pi_{1},\left(A_{\ell+n+1},\left\{\gamma_{1}, \gamma_{\ell+1}\right\}\right)\right](n \geqq 0, \ell \geqq 1)$.

$\mathfrak{b}=\mathfrak{b}_{-1} \oplus \mathfrak{b}_{0} \oplus \mathfrak{b}_{1}$ is described by $\left(A_{\ell+n+1},\left\{\gamma_{\ell+1}\right\}\right)$ and $\check{\mathfrak{g}}=\bigoplus_{p=-\mu}^{\mu} \check{\mathfrak{g}}_{p}$ is described by $\left(A_{\ell+n+1},\left\{\gamma_{1}, \gamma_{\ell+1}\right\}\right)$. Hence $\mu=2$ and we obtain $\check{\mathfrak{g}}=\mathfrak{g}^{2}(\ell, n+1)$. The matrix representation is given as $(1)$ in $\S 2.1$.

We have $S_{-2}=W \cong M(n+1,1), \mathfrak{l}_{-1}=V \cong M(\ell, 1), S_{-1}=W \otimes V^{*} \cong M(n+1, \ell)$ and $\mathfrak{g}_{0}$ determines the splitting of $\mathfrak{g}_{-1}=S_{-1} \oplus \mathfrak{l}_{-1}$. Thus the model equation of our typical symbol $\mathfrak{m}=\mathfrak{C}^{1}(V, W)$ is given by

$$
\frac{\partial^{2} y^{\alpha}}{\partial x_{p} \partial x_{q}}=o \quad \text { for } \quad \alpha=1, \ldots, n+1, \quad 1 \leqq p, q \leqq \ell,
$$

where $y^{1}, \ldots, y^{n+1}$ are dependent variables and $x_{1}, \ldots, x_{\ell}$ are independent variables.

Case $(3)\left[\left(A_{\ell},\left\{\alpha_{i}\right\}\right), 2 \varpi_{l},\left(C_{\ell+1},\left\{\gamma_{i}, \gamma_{\ell+1}\right\}\right)\right](1<i \leqq \ell, \ell \geqq 2)$.

$\mathfrak{b}=\mathfrak{b}_{-1} \oplus \mathfrak{b}_{0} \oplus \mathfrak{b}_{1}$ is described by $\left(C_{\ell+1},\left\{\gamma_{\ell+1}\right\}\right)$ and $\check{\mathfrak{g}}=\bigoplus_{p=-\mu}^{\mu} \check{\mathfrak{g}}_{p}$ is described by $\left(C_{\ell+1},\left\{\gamma_{i}, \gamma_{\ell+1}\right\}\right)$. Hence $\mu=3$ and $\check{\mathfrak{g}}=\mathfrak{b}$ is isomorphic to $\mathfrak{s p}(\ell+1, \mathbb{K})$. First we describe

$$
\mathfrak{s p}(\ell+1, \mathbb{K})=\left\{X \in \mathfrak{g l}(2 \ell+2, \mathbb{K}) \mid{ }^{t} X J+J X=0\right\},
$$

where

$$
J=\left(\begin{array}{cccc}
0 & 0 & 0 & I_{i} \\
0 & 0 & I_{j} & 0 \\
0 & -I_{j} & 0 & 0 \\
-I_{i} & 0 & 0 & 0
\end{array}\right) \in \mathfrak{g l}(2 \ell+2, \mathbb{K}), \quad I_{k}=\left(\delta_{p q}\right) \in \mathfrak{g l}(k, \mathbb{K})
$$


Here $I_{k} \in \mathfrak{g l}(k, \mathbb{K})$ is the unit matrix and the gradation is given again by subdividing matrices as follows;

$$
\begin{aligned}
& \mathfrak{g}_{-3}=S_{-3}=S^{2}\left(T_{0}^{*}\right)=\left\{\left(\begin{array}{cccc}
0 & 0 & 0 & 0 \\
0 & 0 & 0 & 0 \\
0 & 0 & 0 & 0 \\
A & 0 & 0 & 0
\end{array}\right) \mid\right. \\
& \mathfrak{g}_{-2}=S_{-2}=T_{-1} \otimes T_{0}^{*}=\left\{\left(\begin{array}{cccc}
0 & 0 & 0 & 0 \\
0 & 0 & 0 & 0 \\
B & 0 & 0 & 0 \\
0 & { }^{t} B & 0 & 0
\end{array}\right) \mid\right.
\end{aligned}
$$

$\mathfrak{g}_{-1}=S_{-1} \oplus \mathfrak{l}_{-1}$,

$$
\begin{aligned}
& S_{-1}=S^{2}\left(T_{-1}^{*}\right)=\left\{\left(\begin{array}{cccc}
0 & 0 & 0 & 0 \\
0 & 0 & 0 & 0 \\
0 & D & 0 & 0 \\
0 & 0 & 0 & 0
\end{array}\right) \mid D \in \operatorname{Sym}(j)\right\} \\
& \mathfrak{l}_{-1}=T_{-1} \otimes T_{0}^{*}=\left\{\left(\begin{array}{cccc}
0 & 0 & 0 & 0 \\
C & 0 & 0 & 0 \\
0 & 0 & 0 & 0 \\
0 & 0 & -{ }^{t} C & 0
\end{array}\right) \mid C \in M(j, i)\right\}, \\
& \mathfrak{g}_{0}=\check{\mathfrak{l}}_{0}=\left\{\left(\begin{array}{cccc}
F & 0 & 0 & 0 \\
0 & G & 0 & 0 \\
0 & 0 & -{ }^{t} G & 0 \\
0 & 0 & 0 & -{ }^{t} F
\end{array}\right) \mid F \in \mathfrak{g l}(i, \mathbb{K}), G \in \mathfrak{g l}(j, \mathbb{K}),\right\} \\
& \check{\mathfrak{g}}_{k}=\left\{{ }^{t} X \mid X \in \mathfrak{g}_{-k}\right\},(k=1,2,3),
\end{aligned}
$$

where $i+j=\ell+1, T=T_{0} \oplus T_{-1}=\mathbb{K}^{\ell+1}, T_{0}=\mathbb{K}^{i}, T_{-1}=\mathbb{K}^{j}$ and $\operatorname{Sym}(k)=\{A \in$ $\left.\mathfrak{g l}(k, \mathbb{K}) \mid{ }^{t} A=A\right\}$ is the space of symmetric matrices. Thus we have

$$
S=S^{2}\left(T^{*}\right), \quad \mathfrak{l}=\mathfrak{s l}(T), \text { and } \quad \check{\mathfrak{g}}=\mathfrak{s p}(T \oplus T) .
$$

We will divide the argument into the following two cases. We first consider the typical case:

(i) $i=\ell \geqq 2$.

We have $j=1$ in the above matrix description. Hence $\operatorname{dim} \mathfrak{l}_{-1}=S_{-2}=\ell, \operatorname{dim} S_{-1}=1$ and $\operatorname{dim} S_{-3}=\frac{1}{2} \ell(\ell+1)$. Then

$$
\begin{aligned}
\mathfrak{m} & =S_{-3} \oplus S_{-2} \oplus\left(S_{-1} \oplus \mathfrak{l}_{-1}\right) \\
& =\left\{\left(\begin{array}{cccc}
0 & 0 & 0 & 0 \\
x & 0 & 0 & 0 \\
\xi & a & 0 & 0 \\
Y & { }^{t} \xi & -{ }^{t} x & 0
\end{array}\right)=\hat{Y}+\check{\xi}+\hat{a}+\hat{x} \mid a \in \mathbb{K}, x, \xi \in \mathbb{K}^{\ell}=M(1, \ell), Y \in \operatorname{Sym}(\ell)\right\},
\end{aligned}
$$

By calculating $[\hat{\xi}, \hat{x}]$ and $[[\hat{a}, \hat{x}], \hat{x}]$, we have

$$
y_{p q}\left(=y_{q p}\right)=\xi_{p} x_{q}+\xi_{q} x_{p}=2 a x_{p} x_{q},
$$


where $Y=\left(y_{p q}\right), \xi=\left(\xi_{1}, \ldots, \xi_{\ell}\right)$ and $x=\left(x_{1}, \ldots, x_{\ell}\right)$. From the first equality, we can embed $S_{-2}$ as a subspace of $S_{-3} \otimes\left(\mathfrak{l}_{-1}\right)^{*}$ and obtain the following first order system as the model equation whose symbol coincides with this subspace:

$$
\frac{\partial y_{p q}}{\partial x_{r}}=0 \quad \text { for } \quad r \neq p, q, \quad \frac{\partial y_{p q}}{\partial x_{q}}=\frac{1}{2} \frac{\partial y_{p p}}{\partial x_{p}} \quad \text { for } \quad p \neq q,
$$

where $y_{p q}=y_{q p}(1 \leqq p \leqq q \leqq \ell)$ are dependent variables and $x_{1}, \ldots, x_{\ell}$ are independent variables. Moreover, by a direct calculation, we see that the prolongation of the first order system (3.6) is given by

$$
\frac{\partial^{2} y_{p q}}{\partial x_{r} \partial x_{s}}=0 \quad \text { for } \quad\{r, s\} \neq\{p, q\}, \quad \frac{\partial^{2} y_{p q}}{\partial x_{p} \partial x_{q}}=\frac{1}{2} \frac{\partial^{2} y_{p p}}{\partial^{2} x_{p}}=\frac{1}{2} \frac{\partial^{2} y_{q q}}{\partial^{2} x_{q}} \quad \text { for } \quad p \neq q
$$

From the second equality, we observe that the above second order system is the model equation of the 1-dimensional embedded subspace $S_{-1}$ in $S_{-3} \otimes S^{2}\left(\left(\mathfrak{l}_{-1}\right)^{*}\right)$. Furthermore, by a direct calculation, we see that the prolongation of this second order system (3.7) is given by

$$
\frac{\partial^{3} y_{p q}}{\partial x_{r} \partial x_{s} \partial x_{t}}=0 \quad \text { for } \quad 1 \leqq p, q, r, s, t \leqq \ell
$$

(ii) $1<i<\ell$.

We have $S_{-3}=S^{2}\left(T_{0}^{*}\right) \cong \operatorname{Sym}(i), S_{-2}=T_{-1} \otimes T_{0}^{*} \cong M(j, i), S_{-1}=S^{2}\left(T_{-1}^{*}\right) \cong \operatorname{Sym}(j)$ and $\mathfrak{l}_{-1}=T_{-1} \otimes T_{0}^{*} \cong M(j, i)$. Then

$$
\begin{aligned}
\mathfrak{m} & =S_{-3} \oplus S_{-2} \oplus\left(S_{-1} \oplus \mathfrak{l}_{-1}\right) \\
& =\left\{\left(\begin{array}{cccc}
0 & 0 & 0 & 0 \\
X & 0 & 0 & 0 \\
\Xi & A & 0 & 0 \\
Y & { }^{t} \Xi & -{ }^{t} X & 0
\end{array}\right)=\hat{Y}+\check{\Xi}+\hat{A}+\hat{X} \mid A \in \operatorname{Sym}(j), X, \Xi \in M(j, i), Y \in \operatorname{Sym}(i)\right\},
\end{aligned}
$$

By calculating $[\hat{\Xi}, \hat{X}]$ and $[[\hat{A}, \hat{X}], \hat{X}]$, we have

$$
y_{p q}\left(=y_{q p}\right)=\sum_{\alpha=1}^{j}\left(\xi_{p}^{\alpha} x_{q}^{\alpha}+\xi_{q}^{\alpha} x_{p}^{\alpha}\right)=2 \sum_{\alpha, \beta=1}^{j} a_{\alpha \beta} x_{p}^{\alpha} x_{q}^{\beta}
$$

where $Y=\left(y_{p q}\right), \Xi=\left(\xi_{p}^{\alpha}\right) A=\left(a_{\alpha \beta}\right)$ and $X=\left(x_{p}^{\alpha}\right)$. From the first equality, we can embed $S_{-2}$ as a subspace of $S_{-3} \otimes\left(\mathfrak{l}_{-1}\right)^{*}$ and obtain the following first order system as the model equation whose symbol coincides with this subspace:

$$
\frac{\partial y_{p q}}{\partial x_{r}^{\alpha}}=0 \quad \text { for } \quad r \neq p, q, \quad \frac{\partial y_{p q}}{\partial x_{q}^{\alpha}}=\frac{1}{2} \frac{\partial y_{p p}}{\partial x_{p}^{\alpha}} \quad \text { for } \quad p \neq q .
$$

where $y_{p q}=y_{q p}(1 \leqq p \leqq q \leqq i)$ are dependent variables and $x_{p}^{\alpha}(1 \leqq p \leqq i, 1 \leqq \alpha \leqq j)$ are independent variables. Moreover, by a direct calculation, we see that the prolongation of the first order system (3.9) is given by

$$
\frac{\partial^{2} y_{p q}}{\partial x_{r}^{\alpha} \partial x_{s}^{\beta}}=0 \quad \text { for }\{r, s\} \neq\{p, q\}, \quad \frac{\partial^{2} y_{p q}}{\partial x_{p}^{\alpha} \partial x_{q}^{\beta}}=\frac{1}{2} \frac{\partial^{2} y_{p p}}{\partial x_{p}^{\alpha} \partial x_{p}^{\beta}}=\frac{1}{2} \frac{\partial^{2} y_{q q}}{\partial x_{q}^{\alpha} \partial x_{q}^{\beta}} \quad \text { for } p \neq q .
$$

From the second equality, we observe that the above second order system is the model equation of the embedded subspace $S_{-1}$ in $S_{-3} \otimes S^{2}\left(\left(\mathfrak{l}_{-1}\right)^{*}\right)$. Furthermore, by a direct 
calculation, we see that the prolongation of this second order system (3.10) is given by

$$
\frac{\partial^{3} y_{p q}}{\partial x_{r}^{\alpha} \partial x_{s}^{\beta} \partial x_{t}^{\gamma}}=0 \quad \text { for } \quad 1 \leqq p, q, r, s, t \leqq i, \quad 1 \leqq \alpha, \beta, \gamma \leqq j
$$

Case $(4)\left[\left(A_{\ell},\left\{\alpha_{1}\right\}\right), 2 \varpi_{l},\left(C_{\ell+1},\left\{\gamma_{1}, \gamma_{\ell+1}\right\}\right)\right](\ell \geqq 1)$.

$\mathfrak{b}=\mathfrak{b}_{-1} \oplus \mathfrak{b}_{0} \oplus \mathfrak{b}_{1}$ is described by $\left(C_{\ell+1},\left\{\gamma_{\ell+1}\right\}\right)$ and $\check{\mathfrak{g}}=\bigoplus_{p=-\mu}^{\mu} \check{\mathfrak{g}}_{p}$ is described by $\left(C_{\ell+1},\left\{\gamma_{1}, \gamma_{\ell+1}\right\}\right)$. Hence $\mu=3$ and we obtain $\check{\mathfrak{g}}=\mathfrak{g}^{3}(\ell, 1)$. The matrix representation is given as (2) in $\S 2.1$.

We have $S_{-3}=\mathbb{K}, S_{-2}=V^{*}, \mathfrak{l}_{-1}=V, S_{-1}=S^{2}(V *)$ and $\mathfrak{g}_{0}$ determines the splitting of $\mathfrak{g}_{-1}=S_{-1} \oplus \mathfrak{l}_{-1}$. Thus the model equation of our typical symbol $\mathfrak{m}=\mathfrak{C}^{2}(V, \mathbb{K})$ is given by

$$
\frac{\partial^{3} y}{\partial x_{p} \partial x_{q} \partial x_{r}}=0 \quad \text { for } \quad 1 \leqq p, q, r \leqq \ell
$$

where $y$ is dependent variable and $x_{1}, \ldots, x_{\ell}$ are independent variables.

Case $(5)\left[\left(B_{\ell},\left\{\alpha_{1}\right\}\right), \varpi_{1},\left(B_{\ell+1},\left\{\gamma_{1}, \gamma_{2}\right\}\right)\right](\ell \geqq 2)\left[\left(D_{\ell},\left\{\alpha_{1}\right\}\right), \varpi_{1},\left(D_{\ell+1},\left\{\gamma_{1}, \gamma_{2}\right\}\right)\right](\ell \geqq 4)$.

$\mathfrak{b}=\mathfrak{b}_{-1} \oplus \mathfrak{b}_{0} \oplus \mathfrak{b}_{1}$ is described by $\left(B D_{\ell+1},\left\{\gamma_{1}\right\}\right)$ and $\check{\mathfrak{g}}=\bigoplus_{p=-\mu}^{\mu} \check{\mathfrak{g}}_{p}$ is described by $\left(B D_{\ell+1},\left\{\gamma_{1}, \gamma_{2}\right\}\right)$. Hence $\mu=3$ and $\check{\mathfrak{g}}=\mathfrak{b}$ is isomorphic to $\mathfrak{o}(n+4)$. First we describe

$$
\mathfrak{o}(n+4)=\left\{X \in \mathfrak{g l}(n+4, \mathbb{K}) \mid{ }^{t} X J+J X=0\right\}
$$

where

$$
J=\left(\begin{array}{ccccc}
0 & 0 & 0 & 0 & 1 \\
0 & 0 & 0 & 1 & 0 \\
0 & 0 & I_{n} & 0 & 0 \\
0 & 1 & 0 & 0 & 0 \\
1 & 0 & 0 & 0 & 0
\end{array}\right) \in \mathfrak{g l}(n+4, \mathbb{K}), \quad I_{n}=\left(\delta_{i j}\right) \in \mathfrak{g l}(n, \mathbb{K})
$$


Here $I_{n} \in \mathfrak{g l}(n, \mathbb{K})$ is the unit matrix and the gradation is given again by subdividing matrices as follows;

$$
\begin{aligned}
& \mathfrak{g}_{-3}=S_{-3}=\left\{\left(\begin{array}{ccccc}
0 & 0 & 0 & 0 & 0 \\
0 & 0 & 0 & 0 & 0 \\
0 & 0 & 0 & 0 & 0 \\
y & 0 & 0 & 0 & 0 \\
0 & -y & 0 & 0 & 0
\end{array}\right) \mid \quad y \in \mathbb{K}\right\} \\
& \mathfrak{g}_{-2}=S_{-2}=\left\{\left(\begin{array}{ccccc}
0 & 0 & 0 & 0 & 0 \\
0 & 0 & 0 & 0 & 0 \\
\xi & 0 & 0 & 0 & 0 \\
0 & 0 & 0 & 0 & 0 \\
0 & 0 & -{ }^{t} \xi & 0 & 0
\end{array}\right) \mid \quad \xi \in \mathbb{K}^{n}=M(n, 1)\right\} \text {, } \\
& \mathfrak{g}_{-1}=S_{-1} \oplus \mathfrak{l}_{-1}, \\
& S_{-1}=\left\{\left(\begin{array}{ccccc}
0 & 0 & 0 & 0 & 0 \\
b & 0 & 0 & 0 & 0 \\
0 & 0 & 0 & 0 & 0 \\
0 & 0 & 0 & 0 & 0 \\
0 & 0 & 0 & -b & 0
\end{array}\right) \mid b \in \mathbb{K}\right\}, \\
& \mathfrak{l}_{-1}=\left\{\left(\begin{array}{ccccc}
0 & 0 & 0 & 0 & 0 \\
0 & 0 & 0 & 0 & 0 \\
0 & x & 0 & 0 & 0 \\
0 & 0 & -{ }^{t} x & 0 & 0 \\
0 & 0 & 0 & 0 & 0
\end{array}\right) \mid x \in \mathbb{K}^{n}=M(n, 1)\right\}, \\
& \mathfrak{g}_{0}=\check{\mathfrak{l}}_{0}=\left\{\left(\begin{array}{ccccc}
a_{1} & 0 & 0 & 0 & 0 \\
0 & a_{2} & 0 & 0 & 0 \\
0 & 0 & A & 0 & 0 \\
0 & 0 & 0 & -a_{2} & 0 \\
0 & 0 & 0 & 0 & -a_{1}
\end{array}\right) \mid a_{1}, a_{2} \in \mathbb{K}, A \in \mathfrak{o}(n)\right\} \\
& \check{\mathfrak{g}}_{k}=\left\{{ }^{t} X \mid X \in \mathfrak{g}_{-k}\right\},(k=1,2,3) \text {. }
\end{aligned}
$$

We have $\operatorname{dim} S_{-3}=\operatorname{dim} S_{-1}=1$ and $\operatorname{dim} S_{-2}=\operatorname{dim} \mathfrak{l}_{-1}=n$. Then

$$
\begin{aligned}
& \mathfrak{m}=S_{-3} \oplus S_{-2} \oplus\left(S_{-1} \oplus \mathfrak{l}_{-1}\right) \\
&=\left\{\left(\begin{array}{ccccc}
0 & 0 & 0 & 0 & 0 \\
a & 0 & 0 & 0 & 0 \\
\xi & x & 0 & 0 & 0 \\
y & 0 & -{ }^{t} x & 0 & 0 \\
0 & -y & -{ }^{t} \xi & -a & 0
\end{array}\right)=\hat{y}+\check{\xi}+\hat{a}+\hat{x}\right. \\
&
\end{aligned}
$$

From $[\check{\xi}, \hat{x}]=\left(\sum_{i=1}^{n} \xi_{i} x_{i}\right)^{\wedge}$ and $[[\hat{a}, \hat{x}], \hat{x}]=\left(-a \sum_{i=1}^{n} x_{i}^{2}\right)^{\wedge}$, we have $S_{-2}=V^{*}$, putting $S_{-3}=\mathbb{K}$ and $\mathfrak{l}_{-1}=V$. Moreover $S_{-1}$ is embedded as the 1-dimensional subspace spanned by the unit matrix in $\operatorname{Sym}(n) \cong S^{2}\left(V^{*}\right)$ through the bracket operation in $\mathfrak{m}$. This implies that the model equation of our typical symbol $\mathfrak{m}=\mathfrak{g}_{-3} \oplus \mathfrak{g}_{-2} \oplus \mathfrak{g}_{-1} \subset \mathfrak{C}^{2}(V, \mathbb{K})$ is given 
by

$$
\frac{\partial^{2} y}{\partial x_{p} \partial x_{q}}=\delta_{p q} \frac{\partial^{2} y}{\partial^{2} x_{1}} \quad \text { for } \quad 1 \leqq p, q \leqq n,
$$

where $y$ is dependent variable and $x_{1}, \ldots, x_{n}$ are independent variables. By a direct calculation, we see that the prolongation of the second order system (3.13) is given by

$$
\frac{\partial^{3} y}{\partial x_{p} \partial x_{q} \partial x_{r}}=0 \quad \text { for } \quad 1 \leqq p, q, r \leqq n .
$$

Case $(6)\left[\left(D_{\ell},\left\{\alpha_{\ell}\right\}\right), \varpi_{1},\left(D_{\ell+1},\left\{\gamma_{1}, \gamma_{\ell+1}\right\}\right)\right](\ell \geqq 4)$.

$\mathfrak{b}=\mathfrak{b}_{-1} \oplus \mathfrak{b}_{0} \oplus \mathfrak{b}_{1}$ is described by $\left(D_{\ell+1},\left\{\gamma_{1}\right\}\right)$ and $\check{\mathfrak{g}}=\bigoplus_{p=-\mu}^{\mu} \check{\mathfrak{g}}_{p}$ is described by $\left(D_{\ell+1},\left\{\gamma_{1}, \gamma_{\ell+1}\right\}\right)$. Hence $\mu=2$ and $\check{\mathfrak{g}}=\mathfrak{b}$ is isomorphic to $\mathfrak{o}(2 \ell+2)$. First we describe

$$
\mathfrak{o}(2 \ell+2)=\left\{X \in \mathfrak{g l}(2 \ell+2, \mathbb{K}) \mid{ }^{t} X J+J X=0\right\},
$$

where

$$
J=\left(\begin{array}{cccc}
0 & 0 & 0 & 1 \\
0 & 0 & I_{\ell} & 0 \\
0 & I_{\ell} & 0 & 0 \\
1 & 0 & 0 & 0
\end{array}\right) \in \mathfrak{g l}(2 \ell+2, \mathbb{K}), \quad I_{\ell}=\left(\delta_{i j}\right) \in \mathfrak{g l}(\ell, \mathbb{K}) .
$$

Here the gradation is given again by subdividing matrices as follows;

$$
\begin{aligned}
& \mathfrak{g}_{-2}=S_{-2}=\left\{\left(\begin{array}{cccc}
0 & 0 & 0 & 0 \\
0 & 0 & 0 & 0 \\
y & 0 & 0 & 0 \\
0 & -{ }^{t} y & 0 & 0
\end{array}\right) \mid \quad y \in \mathbb{K}^{\ell}=M(\ell, 1)\right\}, \\
& \mathfrak{g}_{-1}=S_{-1} \oplus \mathfrak{l}_{-1}, \\
& S_{-1}=\left\{\left(\begin{array}{cccc}
0 & 0 & 0 & 0 \\
\xi & 0 & 0 & 0 \\
0 & 0 & 0 & 0 \\
0 & 0 & -{ }^{t} \xi & 0
\end{array}\right) \mid \xi \in \mathbb{K}^{\ell}=M(\ell, 1)\right\} \\
& \mathfrak{l}_{-1}=\left\{\left(\begin{array}{cccc}
0 & 0 & 0 & 0 \\
0 & 0 & 0 & 0 \\
0 & X & 0 & 0 \\
0 & 0 & 0 & 0
\end{array}\right) \mid X \in \mathfrak{o}(\ell)\right\} \\
& \mathfrak{g}_{0}=\check{\mathfrak{l}}_{0}=\left\{\left(\begin{array}{cccc}
a & 0 & 0 & 0 \\
0 & A & 0 & 0 \\
0 & 0 & -{ }^{t} A & 0 \\
0 & 0 & 0 & -a
\end{array}\right) \mid a \in \mathbb{K}, A \in \mathfrak{g l}(\ell, \mathbb{K}),\right\} \\
& \check{\mathfrak{g}}_{k}=\left\{{ }^{t} X \mid X \in \mathfrak{g}_{-k}\right\},(k=1,2,3),
\end{aligned}
$$

We have $\operatorname{dim} S_{-2}=\operatorname{dim} S_{-1}=\ell, \operatorname{dim} \mathfrak{l}_{-1}=\frac{1}{2} \ell(\ell-1), \mathfrak{l}=\mathfrak{o}(2 \ell)$ and $S=\mathbb{K}^{2 \ell}$. Then

$$
\begin{aligned}
\mathfrak{m} & =S_{-2} \oplus\left(S_{-1} \oplus \mathfrak{l}_{-1}\right) \\
& =\left\{\left(\begin{array}{cccc}
0 & 0 & 0 & 0 \\
\xi & 0 & 0 & 0 \\
y & X & 0 & 0 \\
0 & -{ }^{t} y & -{ }^{t} \xi & 0
\end{array}\right)=\hat{y}+\check{\xi}+\hat{X} \mid y, \xi \in \mathbb{K}^{\ell}=M(\ell, 1), X \in \mathfrak{o}(\ell)\right\} .
\end{aligned}
$$


By calculating $[\check{\xi}, \hat{X}]$, we have

$$
y_{p}=\sum_{q=1}^{\ell} x_{p q} \xi_{q}, \quad\left(x_{p q}+x_{q p}=0\right),
$$

where $y={ }^{t}\left(y_{1}, \ldots, y_{\ell}\right), \xi={ }^{t}\left(\xi_{1}, \ldots, \xi_{\ell}\right)$ and $X=\left(x_{p q}\right)$. Then the model equation of our typical symbol $\mathfrak{m}=\mathfrak{g}_{-2} \oplus \mathfrak{g}_{-1} \subset \mathfrak{C}^{1}\left(\mathfrak{l}_{-1}, S_{-2}\right)$ is given by

$$
\frac{\partial y_{p}}{\partial x_{q r}}=0 \quad \text { for distinct } \quad p, q, r \quad \frac{\partial y_{p}}{\partial x_{p q}}=\frac{\partial y_{r}}{\partial x_{r q}} \quad \text { for } \quad p, r \neq q
$$

where $y_{1}, \ldots, y_{\ell}$ are dependent variables and $x_{p q}(1 \leqq p<q \leqq \ell)$ are independent variables. By a direct calculation, we see that the prolongation of the first order system (3.15) is given by

$$
\frac{\partial^{2} y_{p}}{\partial x_{q_{1} r_{1}} \partial x_{q_{2} r_{2}}}=0 \quad \text { for } \quad 1 \leqq p, q_{1}, r_{1}, q_{2}, r_{2} \leqq \ell
$$

Case $(7)\left[\left(A_{\ell},\left\{\alpha_{1}\right\}, \varpi_{\ell-1},\left(D_{\ell+1},\left\{\gamma_{1}, \gamma_{\ell+1}\right\}\right)\right](\ell \geqq 3)\right.$.

$\mathfrak{b}=\mathfrak{b}_{-1} \oplus \mathfrak{b}_{0} \oplus \mathfrak{b}_{1}$ is described by $\left(D_{\ell+1},\left\{\gamma_{\ell+1}\right\}\right)$ and $\check{\mathfrak{g}}=\bigoplus_{p=-\mu}^{\mu} \check{\mathfrak{g}}_{p}$ is described by $\left(D_{\ell+1},\left\{\gamma_{1}, \gamma_{\ell+1}\right\}\right)$. Hence $\mu=2$ and $\check{\mathfrak{g}}=\mathfrak{b}$ is isomorphic to $\mathfrak{o}(2 \ell+2)$. First we describe

$$
\mathfrak{o}(2 \ell+2)=\left\{X \in \mathfrak{g l}(2 \ell+2, \mathbb{K}) \mid{ }^{t} X J+J X=0\right\},
$$

as in Case (6) and the gradation is given again by subdividing matrices as follows;

$$
\begin{aligned}
& \mathfrak{g}_{-2}=S_{-2}=\left\{\left(\begin{array}{cccc}
0 & 0 & 0 & 0 \\
0 & 0 & 0 & 0 \\
y & 0 & 0 & 0 \\
0 & -{ }^{t} y & 0 & 0
\end{array}\right) \mid \quad y \in \mathbb{K}^{\ell}=M(\ell, 1)\right\}, \\
& \mathfrak{g}_{-1}=S_{-1} \oplus \mathfrak{l}_{-1}, \\
& S_{-1}=\left\{\left(\begin{array}{cccc}
0 & 0 & 0 & 0 \\
0 & 0 & 0 & 0 \\
0 & \Xi & 0 & 0 \\
0 & 0 & 0 & 0
\end{array}\right) \mid \Xi \in \mathfrak{o}(\ell)\right\}, \\
& \mathfrak{l}_{-1}=\left\{\left(\begin{array}{cccc}
0 & 0 & 0 & 0 \\
x & 0 & 0 & 0 \\
0 & 0 & 0 & 0 \\
0 & 0 & -{ }^{t} x & 0
\end{array}\right) \mid x \in \mathbb{K}^{\ell}=M(\ell, 1)\right\}, \\
& \mathfrak{g}_{0}=\check{\mathfrak{l}}_{0}=\left\{\left(\begin{array}{cccc}
a & 0 & 0 & 0 \\
0 & A & 0 & 0 \\
0 & 0 & -{ }^{t} A & 0 \\
0 & 0 & 0 & -a
\end{array}\right) \mid a \in \mathbb{K}, A \in \mathfrak{g l}(\ell, \mathbb{K}),\right\} \\
& \check{\mathfrak{g}}_{k}=\left\{{ }^{t} X \mid X \in \mathfrak{g}_{-k}\right\},(k=1,2,3),
\end{aligned}
$$


We have $\operatorname{dim} S_{-2}=\operatorname{dim} \mathfrak{l}_{-1}=\ell, \operatorname{dim} S_{-1}=\frac{1}{2} \ell(\ell-1), \mathfrak{l}=\mathfrak{s l}(T)$ and $S=\bigwedge^{2} T^{*},$. Then

$$
\begin{aligned}
\mathfrak{m} & =S_{-2} \oplus\left(S_{-1} \oplus \mathfrak{l}_{-1}\right) \\
& =\left\{\left(\begin{array}{cccc}
0 & 0 & 0 & 0 \\
x & 0 & 0 & 0 \\
y & \Xi & 0 & 0 \\
0 & -{ }^{t} y & -{ }^{t} x & 0
\end{array}\right)=\check{y}+\hat{\Xi}+\hat{x} \mid x, y \in \mathbb{K}^{\ell}=M(\ell, 1), \Xi \in \mathfrak{o}(\ell)\right\} .
\end{aligned}
$$

By calculating $[\hat{\Xi}, \hat{x}]$, we have

$$
y_{p}=\sum_{q=1}^{\ell} \xi_{p q} x_{q}, \quad\left(\xi_{p q}+\xi_{q p}=0\right),
$$

where $y={ }^{t}\left(y_{1}, \ldots, y_{\ell}\right), x={ }^{t}\left(x_{1}, \ldots, x_{\ell}\right)$ and $\Xi=\left(\xi_{p q}\right)$. Then the model equation of our typical symbol $\mathfrak{m}=\mathfrak{g}_{-2} \oplus \mathfrak{g}_{-1} \subset \mathfrak{C}^{1}\left(\mathfrak{l}_{-1}, S_{-2}\right)$ is given by

$$
\frac{\partial y_{p}}{\partial x_{q}}+\frac{\partial y_{q}}{\partial x_{p}}=0 \quad \text { for } \quad 1 \leqq p<q \leqq \ell,
$$

where $y_{1}, \ldots, y_{\ell}$ are dependent variables and $x_{1}, \ldots, x_{\ell}$ are independent variables. By a direct calculation, we see that the prolongation of the first order system (3.17) is given by

$$
\frac{\partial^{2} y_{p}}{\partial x_{q} \partial x_{r}}=0 \quad \text { for } \quad 1 \leqq p, q, r \leqq \ell
$$

Case $(8)\left[\left(A_{\ell},\left\{\alpha_{\ell}\right\}\right), \varpi_{\ell-1},\left(D_{\ell+1},\left\{\gamma_{\ell}, \gamma_{\ell+1}\right\}\right)\right](\ell \geqq 3)$.

$\mathfrak{b}=\mathfrak{b}_{-1} \oplus \mathfrak{b}_{0} \oplus \mathfrak{b}_{1}$ is described by $\left(D_{\ell+1},\left\{\gamma_{\ell+1}\right\}\right)$ and $\check{\mathfrak{g}}=\bigoplus_{p=-\mu}^{\mu} \check{\mathfrak{g}}_{p}$ is described by $\left(D_{\ell+1},\left\{\gamma_{\ell}, \gamma_{\ell+1}\right\}\right)$. Hence $\mu=2$ and $\check{\mathfrak{g}}=\mathfrak{b}$ is isomorphic to $\mathfrak{o}(2 \ell+2)$. First we describe

$$
\mathfrak{o}(2 \ell+2)=\left\{X \in \mathfrak{g l}(2 \ell+2, \mathbb{K}) \mid{ }^{t} X J+J X=0\right\},
$$

where

$$
J=\left(\begin{array}{cccc}
0 & 0 & 0 & I_{\ell} \\
0 & 0 & 1 & 0 \\
0 & 1 & 0 & 0 \\
I_{\ell} & 0 & 0 & 0
\end{array}\right) \in \mathfrak{g l}(2 \ell+2, \mathbb{K}), \quad I_{\ell}=\left(\delta_{i j}\right) \in \mathfrak{g l}(\ell, \mathbb{K})
$$


Here the gradation is given again by subdividing matrices as follows;

$$
\begin{aligned}
& \mathfrak{g}_{-2}=S_{-2}=\left\{\left(\begin{array}{cccc}
0 & 0 & 0 & 0 \\
0 & 0 & 0 & 0 \\
0 & 0 & 0 & 0 \\
Y & 0 & 0 & 0
\end{array}\right) \mid \quad Y \in \mathfrak{o}(\ell)\right\}, \\
& \mathfrak{g}_{-1}=S_{-1} \oplus \mathfrak{l}_{-1}, \\
& S_{-1}=\left\{\left(\begin{array}{cccc}
0 & 0 & 0 & 0 \\
0 & 0 & 0 & 0 \\
\xi & 0 & 0 & 0 \\
0 & -{ }^{t} \xi & 0 & 0
\end{array}\right) \mid \xi \in M(1, \ell)\right\} \\
& \mathfrak{l}_{-1}=\left\{\left(\begin{array}{cccc}
0 & 0 & 0 & 0 \\
x & 0 & 0 & 0 \\
0 & 0 & 0 & 0 \\
0 & 0 & -{ }^{t} x & 0
\end{array}\right) \mid x \in \mathbb{K}^{\ell}=M(1, \ell)\right\}, \\
& \mathfrak{g}_{0}=\check{\mathfrak{l}}_{0}=\left\{\left(\begin{array}{cccc}
A & 0 & 0 & 0 \\
0 & a & 0 & 0 \\
0 & 0 & -a & 0 \\
0 & 0 & 0 & -{ }^{t} A
\end{array}\right) \mid a \in \mathbb{K}, A \in \mathfrak{g l}(\ell, \mathbb{K}),\right\} \\
& \check{\mathfrak{g}}_{k}=\left\{{ }^{t} X \mid X \in \mathfrak{g}_{-k}\right\},(k=1,2,3),
\end{aligned}
$$

We have $\operatorname{dim} S_{-2}=\frac{1}{2} \ell(\ell-1), \operatorname{dim} S_{-1}=\operatorname{dim} \mathfrak{l}_{-1}=\ell, \mathfrak{l}=\mathfrak{s l}(T)$ and $S=\bigwedge^{2} T^{*}$. Then

$$
\begin{aligned}
\mathfrak{m} & =S_{-2} \oplus\left(S_{-1} \oplus \mathfrak{l}_{-1}\right) \\
& =\left\{\left(\begin{array}{cccc}
0 & 0 & 0 & 0 \\
x & 0 & 0 & 0 \\
\xi & 0 & 0 & 0 \\
Y & -{ }^{t} \xi & -{ }^{t} x & 0
\end{array}\right)=\hat{Y}+\check{\xi}+\hat{x} \mid x, \xi \in \mathbb{K}^{\ell}=M(1, \ell), Y \in \mathfrak{o}(\ell)\right\} .
\end{aligned}
$$

By calculating $[\check{\xi}, \hat{x}]$, we have

$$
y_{p q}=\xi_{q} x_{p}-\xi_{p} x_{q}, \quad\left(y_{p q}+y_{q p}=0\right),
$$

where $x=\left(x_{1}, \ldots, x_{\ell}\right), \xi=\left(\xi_{1}, \ldots, \xi_{\ell}\right)$ and $Y=\left(y_{p q}\right)$. Then the model equation of our typical symbol $\mathfrak{m}=\mathfrak{g}_{-2} \oplus \mathfrak{g}_{-1} \subset \mathfrak{C}^{1}\left(\mathfrak{l}_{-1}, S_{-2}\right)$ is given by

$$
\frac{\partial y_{p q}}{\partial x_{r}}=0 \quad \text { for distinct } \quad p, q, r \quad \frac{\partial y_{p q}}{\partial x_{p}}+\frac{\partial y_{q r}}{\partial x_{r}}=0 \quad \text { for } \quad q \neq p, r,
$$

where $y_{p q}(1 \leqq p<q \leqq \ell)$ are dependent variables and $x_{1}, \ldots, x_{\ell}$ are independent variables. By a direct calculation, we see that the prolongation of the first order system (3.19) is given by

$$
\frac{\partial^{2} y_{p q}}{\partial x_{r} \partial x_{s}}=0 \quad \text { for } \quad 1 \leqq p, q, r, s \leqq \ell
$$

Case $(9)\left[\left(A_{\ell},\left\{\alpha_{i}\right\}\right), \varpi_{\ell-1},\left(D_{\ell+1},\left\{\gamma_{i}, \gamma_{\ell+1}\right\}\right)\right](2<i<\ell, \ell \geqq 4)$.

$\mathfrak{b}=\mathfrak{b}_{-1} \oplus \mathfrak{b}_{0} \oplus \mathfrak{b}_{1}$ is described by $\left(D_{\ell+1},\left\{\gamma_{\ell+1}\right\}\right)$ and $\check{\mathfrak{g}}=\bigoplus_{p=-\mu}^{\mu} \check{\mathfrak{g}}_{p}$ is described by $\left(D_{\ell+1},\left\{\gamma_{i}, \gamma_{\ell+1}\right\}\right)$. Hence $\mu=3$ and $\check{\mathfrak{g}}=\mathfrak{b}$ is isomorphic to $\mathfrak{o}(2 \ell+2)$. First we describe

$$
\mathfrak{o}(2 \ell+2)=\left\{\left.X \in \mathfrak{g l}(2 \ell+2, \mathbb{K})\right|^{t} X J+J X=0\right\},
$$


where

$$
J=\left(\begin{array}{cccc}
0 & 0 & 0 & I_{i} \\
0 & 0 & I_{j} & 0 \\
0 & I_{j} & 0 & 0 \\
I_{i} & 0 & 0 & 0
\end{array}\right) \in \mathfrak{g l}(2 \ell+2, \mathbb{K}), \quad I_{k}=\left(\delta_{p q}\right) \in \mathfrak{g l}(k, \mathbb{K}) .
$$

Here the gradation is given again by subdividing matrices as follows;

$$
\begin{aligned}
& \mathfrak{g}_{-3}=S_{-3}=\left\{\left(\begin{array}{cccc}
0 & 0 & 0 & 0 \\
0 & 0 & 0 & 0 \\
0 & 0 & 0 & 0 \\
Y & 0 & 0 & 0
\end{array}\right) \mid \quad Y \in \mathfrak{o}(i)\right\}, \\
& \mathfrak{g}_{-2}=S_{-2}=\left\{\left(\begin{array}{cccc}
0 & 0 & 0 & 0 \\
0 & 0 & 0 & 0 \\
\Xi & 0 & 0 & 0 \\
0 & -{ }^{t} \Xi & 0 & 0
\end{array}\right) \mid \quad \Xi \in M(j, i)\right\} \\
& \mathfrak{g}_{-1}=S_{-1} \oplus \mathfrak{l}_{-1}, \\
& S_{-1}=\left\{\left(\begin{array}{cccc}
0 & 0 & 0 & 0 \\
0 & 0 & 0 & 0 \\
0 & A & 0 & 0 \\
0 & 0 & 0 & 0
\end{array}\right) \mid A \in \mathfrak{o}(j)\right\} \\
& \mathfrak{l}_{-1}=\left\{\left(\begin{array}{cccc}
0 & 0 & 0 & 0 \\
X & 0 & 0 & 0 \\
0 & 0 & 0 & 0 \\
0 & 0 & -{ }^{t} X & 0
\end{array}\right) \mid X \in M(j, i)\right\} \text {, } \\
& \mathfrak{g}_{0}=\check{\mathfrak{l}}_{0}=\left\{\left(\begin{array}{cccc}
F & 0 & 0 & 0 \\
0 & G & 0 & 0 \\
0 & 0 & -{ }^{t} G & 0 \\
0 & 0 & 0 & -{ }^{t} F
\end{array}\right) \mid F \in \mathfrak{g l}(i, \mathbb{K}), G \in \mathfrak{g l}(j, \mathbb{K}),\right\} \\
& \check{\mathfrak{g}}_{k}=\left\{{ }^{t} X \mid X \in \mathfrak{g}_{-k}\right\},(k=1,2,3),
\end{aligned}
$$

We have $i+j=\ell+1, \operatorname{dim} S_{-3}=\frac{1}{2} i(i-1), \operatorname{dim} S_{-2}=\operatorname{dim} \mathfrak{l}_{-1}=i j, \operatorname{dim} S_{-1}=\frac{1}{2} j(j-1)$, $\mathfrak{l}=\mathfrak{s l}(T)$ and $S=\bigwedge^{2} T^{*}$. Then

$$
\begin{aligned}
\mathfrak{m} & =S_{-3} \oplus S_{-2} \oplus\left(S_{-1} \oplus \mathfrak{l}_{-1}\right) \\
& =\left\{\left(\begin{array}{cccc}
0 & 0 & 0 & 0 \\
X & 0 & 0 & 0 \\
\Xi & A & 0 & 0 \\
Y & -{ }^{t} \Xi & -{ }^{t} X & 0
\end{array}\right)=\hat{Y}+\check{\Xi}+\hat{A}+\hat{X} \mid X, \Xi \in M(j, i), Y \in \mathfrak{o}(i), A \in \mathfrak{o}(j)\right\} .
\end{aligned}
$$

By calculating $[\check{\Xi}, \hat{X}]$ and $[[\hat{A}, \hat{X}], \hat{X}]$, we have

$$
y_{p q}\left(=-y_{q p}\right)=\sum_{\alpha=1}^{j}\left(\xi_{q}^{\alpha} x_{p}^{\alpha}-\xi_{p}^{\alpha} x_{q}^{\alpha}\right)=2 \sum_{\alpha, \beta=1}^{j} a_{\alpha \beta} x_{p}^{\alpha} x_{q}^{\beta},
$$

where $Y=\left(y_{p q}\right), \Xi=\left(\xi_{p}^{\alpha}\right)$ and $X=\left(x_{p}^{\alpha}\right)$. From the first equality, we can embed $S_{-2}$ as a subspace of $S_{-3} \otimes\left(\mathfrak{l}_{-1}\right)^{*}$ and obtain the following first order system as the model equation 
whose symbol coincides with this subspace:

$$
\frac{\partial y_{p q}}{\partial x_{r}^{\alpha}}=0 \quad \text { for distinct } \quad p, q, r, \quad \frac{\partial y_{p q}}{\partial x_{p}^{\alpha}}+\frac{\partial y_{q r}}{\partial x_{r}^{\alpha}}=0 \quad \text { for } \quad q \neq p, r,
$$

where $y_{p q}(1 \leqq p<q \leqq i)$ are dependent variables and $\left.x_{p}^{\alpha} 1 \leqq p \leqq i, 1 \leqq \alpha \leqq j\right)$ are independent variables. Moreover, by a direct calculation, we see that the prolongation of the first order system (3.21) is given by

$$
\frac{\partial^{2} y_{p q}}{\partial x_{r}^{\alpha} \partial x_{s}^{\beta}}=\frac{\partial^{2} y_{p q}}{\partial x_{p}^{\alpha} \partial x_{p}^{\beta}}=0 \text { for }\{r, s\} \neq\{p, q\}, \frac{\partial^{2} y_{p q}}{\partial x_{p}^{\alpha} \partial x_{q}^{\beta}}=\frac{\partial^{2} y_{r s}}{\partial x_{r}^{\alpha} \partial x_{s}^{\beta}} \text { for }(p, q) \neq(r, s) \text {. }
$$

From the second equality, we observe that the above second order system is the model equation of the embedded subspace $S_{-1}$ in $S_{-3} \otimes S^{2}\left(\left(\mathfrak{l}_{-1}\right)^{*}\right)$. Furthermore, by a direct calculation, we see that the prolongation of this second order system (3.22) is given by

$$
\frac{\partial^{3} y_{p q}}{\partial x_{r}^{\alpha} \partial x_{s}^{\beta} \partial x_{t}^{\gamma}}=0 \quad \text { for } \quad 1 \leqq p, q, r, s, t \leqq i, \quad 1 \leqq \alpha, \beta, \gamma \leqq j .
$$

Case $(10)\left[\left(A_{\ell},\left\{\alpha_{2}\right\}\right), \varpi_{\ell-1},\left(D_{\ell+1},\left\{\gamma_{2}, \gamma_{\ell+1}\right\}\right)\right](\ell \geqq 3)$.

$\mathfrak{b}=\mathfrak{b}_{-1} \oplus \mathfrak{b}_{0} \oplus \mathfrak{b}_{1}$ is described by $\left(D_{\ell+1},\left\{\gamma_{\ell+1}\right\}\right)$ and $\check{\mathfrak{g}}=\bigoplus_{p=-\mu}^{\mu} \check{\mathfrak{g}}_{p}$ is described by $\left(D_{\ell+1},\left\{\gamma_{2}, \gamma_{\ell+1}\right\}\right)$. Hence $\mu=3$ and $\check{\mathfrak{g}}=\mathfrak{b}$ is isomorphic to $\mathfrak{o}(2 \ell+2)$. First we describe

$$
\mathfrak{o}(2 \ell+2)=\left\{X \in \mathfrak{g l}(2 \ell+2, \mathbb{K}) \mid{ }^{t} X J+J X=0\right\},
$$

where

$$
J=\left(\begin{array}{cccc}
0 & 0 & 0 & I_{2} \\
0 & 0 & I_{\ell-1} & 0 \\
0 & I_{\ell-1} & 0 & 0 \\
I_{2} & 0 & 0 & 0
\end{array}\right) \in \mathfrak{g l}(2 \ell+2, \mathbb{K}), \quad I_{\ell-1}=\left(\delta_{i j}\right) \in \mathfrak{g l}(\ell-1, \mathbb{K}) .
$$

Hence the gradation is given as in the case (9) with $i=2$ and $j=\ell-1$. We have $\operatorname{dim} S_{-3}=1, \operatorname{dim} S_{-2}=\operatorname{dim} \mathfrak{l}_{-1}=2(\ell-1), \operatorname{dim} S_{-1}=\frac{1}{2}(\ell-1)(\ell-2), \mathfrak{l}=\mathfrak{s l}(T)$ and $S=\bigwedge^{2} T^{*}$. Then

$$
\begin{aligned}
\mathfrak{m} & =S_{-3} \oplus S_{-2} \oplus\left(S_{-1} \oplus \mathfrak{l}_{-1}\right) \\
& =\left\{\left(\begin{array}{cccc}
0 & 0 & 0 & 0 \\
X & 0 & 0 & 0 \\
\Xi & A & 0 & 0 \\
Y & -{ }^{t} \Xi & -{ }^{t} X & 0
\end{array}\right)=\hat{Y}+\check{\Xi}+\hat{A}+\hat{X} \mid X, \Xi \in M(\ell-1,2), Y \in \mathfrak{o}(2), A \in \mathfrak{o}(\ell-1)\right\} .
\end{aligned}
$$

By calculating $[\check{\Xi}, \hat{X}]$ and $[[\hat{A}, \hat{X}], \hat{X}]$, we have

$$
y=\sum_{\alpha=1}^{\ell-1}\left(\xi_{1}^{\alpha} x_{2}^{\alpha}+\xi_{2}^{\alpha} x_{1}^{\alpha}\right)=2 \sum_{\alpha, \beta=1}^{\ell-1} a_{\alpha \beta} x_{1}^{\alpha} x_{2}^{\beta},
$$

where $Y=\left(\begin{array}{cc}y & 0 \\ 0 & -y\end{array}\right), A=\left(a_{\alpha \beta}\right)\left(a_{\alpha \beta}+a_{\beta \alpha}=0\right), \Xi=\left(\xi_{p}^{\alpha}\right)$ and $X=\left(x_{p}^{\alpha}\right)$. From the first equality, we have $S_{-2}=V^{*}$, putting $S_{-3}=\mathbb{K}$ and $\mathfrak{l}_{-1}=V$. Moreover, from the second equality and $a_{\alpha \beta}+a_{\beta \alpha}=0$, we see that the model equation of our typical symbol $\mathfrak{m}=\mathfrak{g}_{-3} \oplus \mathfrak{g}_{-2} \oplus \mathfrak{g}_{-1} \subset \mathfrak{C}^{2}(V, \mathbb{K})$ is given by

$$
\frac{\partial^{2} y}{\partial x_{i}^{\alpha} \partial x_{j}^{\beta}}+\frac{\partial^{2} y}{\partial x_{i}^{\beta} \partial x_{j}^{\alpha}}=0 \quad \text { for } \quad 1 \leqq i, j \leqq 2, \quad 1 \leqq \alpha<\beta \leqq \ell-1,
$$


where $y$ are dependent variable and $x_{1}^{1}, \ldots, x_{1}^{\ell-1}, x_{2}^{1}, \ldots, x_{2}^{\ell-1}$ are independent variables. By a direct calculation, we see that the prolongation of the second order system (3.24) is given by

$$
\frac{\partial^{3} y}{\partial x_{i}^{\alpha} \partial x_{j}^{\beta} \partial x_{k}^{\gamma}}=0 \quad \text { for } \quad 1 \leq i, j, k \leqq 2 \quad 1 \leqq \alpha, \beta, \gamma \leqq \ell-1
$$

\section{Symbol of the exceptional CASES}

In this section we will describe the symbol algebra $\mathfrak{m}=\bigoplus_{p<0} \mathfrak{g}_{p}$ explicitly as the subalgebra of $\mathfrak{C}^{\mu-1}(V, W)$, where $V=\mathfrak{l}_{-1}$ and $W=S_{-\mu}$, by first describing the structure of $\mathfrak{m}=\bigoplus_{p<0} \mathfrak{g}_{p}$ explicitly by use of the Chevalley basis of $Y_{L}$.

We first recall that the graded Lie algebra $\check{\mathfrak{g}}=\bigoplus_{p \in \mathbb{Z}} \check{\mathfrak{g}}_{p}$ of type $\left(Y_{L}, \Sigma_{1}\right)$ is described in terms of the root space decomposition as follows (cf. $\S 3$ in [Yam93]): Let us fix a Cartan subalgebra $\mathfrak{h}$ of $\check{\mathfrak{g}}$ and choose a simple root system $\Sigma=\left\{\gamma_{1}, \ldots, \gamma_{L}\right\}$ of the root system $\Phi$ of $\mathfrak{\mathfrak { g }}$ relative to $\mathfrak{h}$. For the subset $\Sigma_{1}$ of $\Sigma$, we put

$$
\Phi_{k}^{+}=\left\{\alpha=\sum_{i=1}^{L} n_{i}(\alpha) \gamma_{i} \in \Phi^{+} \mid \sum_{\gamma_{i} \in \Sigma_{1}} n_{i}(\alpha)=k\right\} \quad \text { for } \quad k \geqq 0,
$$

where $\Phi^{+}$denotes the set of positive roots. Then the gradation $\check{\mathfrak{g}}=\bigoplus_{p \in \mathbb{Z}} \check{\mathfrak{g}}_{p}$ is given by

$$
\check{\mathfrak{g}}_{-k}=\bigoplus_{\alpha \in \Phi_{k}^{+}} \mathfrak{g}_{-\alpha}, \quad \check{\mathfrak{g}}_{0}=\mathfrak{h} \oplus \bigoplus_{\alpha \in \Phi_{0}^{+}}\left(\mathfrak{g}_{\alpha} \oplus \mathfrak{g}_{-\alpha}\right), \quad \check{\mathfrak{g}}_{k}=\bigoplus_{\alpha \in \Phi_{k}^{+}} \mathfrak{g}_{\alpha} \quad(k>0),
$$

where $\mathfrak{g}_{\alpha}$ is the root space for $\alpha \in \Phi$.

In the following, let us take a Chevalley basis $\left\{x_{\alpha}(\alpha \in \Phi) ; h_{i}(1 \leqq i \leqq L)\right\}$ of $\check{\mathfrak{g}}$ and put $y_{\beta}=x_{-\beta}$ for $\beta \in \Phi^{+}$(cf.Chapter VII [Hum72]). We will describe the structure of the negative part $\mathfrak{m}=\sum_{p<0} \check{\mathfrak{g}}_{p}$ of $\left(Y_{L}, \Sigma_{1}\right)$ in terms of $\left\{y_{\beta}\right\}_{\beta \in \Phi^{+}}$. For the property of the Chevalley basis, we recall that, for $\alpha, \beta \in \Phi^{+}$, if $\alpha+\beta \in \Phi$ and $\alpha-\beta \notin \Phi$, then $\left[y_{\alpha}, y_{\beta}\right]= \pm y_{\alpha+\beta}($ see $\S 25.2$ in $[\operatorname{Hum} 72])$.

In this section, we shall treat both complex simple graded Lie algebras $\left(Y_{L}, \Sigma_{1}\right)$ and their normal real forms at the same time and we shall discuss in the complex analytic or the real $C^{\infty}$ category depending on whether we treat complex simple graded Lie algebras $\left(Y_{L}, \Sigma_{1}\right)$ or their normal real forms.

Case (1) $\left[\left(D_{5},\left\{\alpha_{1}\right\}, \varpi_{5},\left(E_{6},\left\{\gamma_{1}, \gamma_{6}\right\}\right)\right]\right.$.

For the gradation of type $\left(E_{6},\left\{\gamma_{1}, \gamma_{6}\right\}\right)$, we have

$$
\begin{aligned}
& \Phi_{2}^{+}=\left\{\quad \alpha_{-7}=111_{0}^{11}, \quad \alpha_{-5}=11111, \quad \alpha_{-3}=11{ }_{1}^{211}, \quad \alpha_{-1}=11221,\right. \\
& \left.\alpha_{1}=12 \underset{1}{2} 11, \quad \alpha_{3}=12 \underset{1}{2} 1, \quad \alpha_{5}=12 \underset{1}{2} 1, \quad \alpha_{7}=12 \underset{2}{2} 21\right\}, \\
& \Phi_{1}^{+}=\Psi^{1} \cup \Psi^{6} \\
& \Psi^{1}=\left\{\quad \xi_{-7}=10 \underset{0}{0} 00, \quad \xi_{-5}=11 \underset{0}{0} 00, \quad \xi_{-3}=11 \underset{0}{100}, \quad \xi_{-1}=11 \underset{0}{110},\right. \\
& \left.\xi_{1}=11 \underset{1}{100}, \quad \xi_{3}=11 \underset{1}{110}, \quad \xi_{5}=11 \underset{1}{210}, \quad \xi_{7}=12 \underset{1}{2} 10 \quad\right\} \text {, } \\
& \Psi^{6}=\left\{\quad \eta_{-7}=\begin{array}{l}
00001 \\
0
\end{array}\right.
\end{aligned}
$$

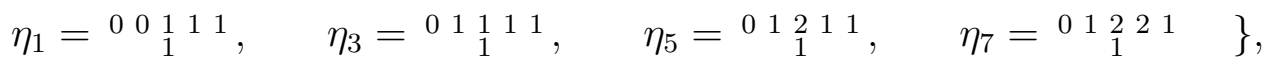




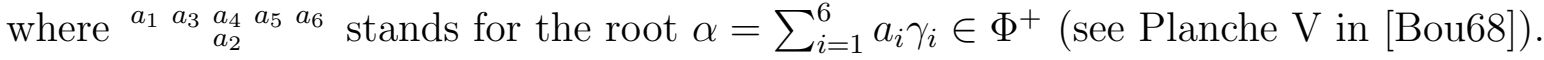

Thus we have $\mu=2$,

$$
\mathfrak{m}=\check{\mathfrak{g}}_{-2} \oplus \check{\mathfrak{g}}_{-1} \quad \text { and } \quad \check{\mathfrak{g}}_{-1}=S_{-1} \oplus \mathfrak{l}_{-1}
$$

where $\check{\mathfrak{g}}_{-2}=S_{-2}, S_{-1}$ and $\mathfrak{l}_{-1}$ are spanned by the root spaces $\mathfrak{g}_{-\beta}$ for $\beta \in \Phi_{2}^{+}, \Psi^{6}$ and $\Psi^{1}$ respectively. Hence $\operatorname{dim} S_{-2}=\operatorname{dim} S_{-1}=\operatorname{dim} \mathfrak{l}_{-1}=8$.

For $\Phi_{2}^{+}, \Psi^{1}$ and $\Psi^{6}$, we observe that $\alpha+\beta \notin \Phi$ for $\alpha, \beta \in \Phi_{2}^{+} \cup \Psi^{1}$ or for $\alpha, \beta \in \Phi_{2}^{+} \cup \Psi^{6}$ and that $\eta-\xi \notin \Phi$ for $\eta \in \Psi^{6}, \xi \in \Psi^{1}$. This implies that $\left[y_{\alpha}, y_{\beta}\right]=0$ for $\alpha, \beta \in \Phi_{2}^{+} \cup \Psi^{1}$ or for $\alpha, \beta \in \Phi_{2}^{+} \cup \Psi^{6}$ and that $\left[y_{\eta}, y_{\xi}\right]= \pm y_{\eta+\xi}$ for $\eta \in \Psi^{6}, \xi \in \Psi^{1}$, if $\eta+\xi \in \Phi$, by the above mentioned property of the Chevalley basis. Hence, from Planche V in [Bou68], we readily obtain the non-trivial bracket relation among $\check{\mathfrak{g}}_{-1}$ as in (4.1) below up to signs.

We solve the problem of signs as follows. First we choose the orientation of $y_{\beta}$ for $\beta \in \Psi^{1}, \Psi^{6}$ and $\Phi_{2}^{+}$as in the following: We choose the orientation of $y_{\gamma_{i}}$ for simple roots by fixing the root vectors $y_{i}=y_{\gamma_{i}} \in \mathfrak{g}_{-\gamma_{i}}$. For $\xi \in \Psi^{1}$, we fix the orientation by the following order;

$$
\begin{aligned}
& y_{\xi_{-7}}=y_{1}, \quad y_{\xi_{-5}}=\left[y_{3}, y_{\xi_{-7}}\right], \quad y_{\xi_{-3}}=\left[y_{4}, y_{\xi_{-5}}\right], \quad y_{\xi_{-1}}=\left[y_{5}, y_{\xi_{-3}}\right], \\
& y_{\xi_{1}}=\left[y_{2}, y_{\xi_{-3}}\right], \quad y_{\xi_{3}}=\left[y_{5}, y_{\xi_{1}}\right], \quad y_{\xi_{5}}=\left[y_{4}, y_{\xi_{3}}\right], \quad y_{\xi_{7}}=\left[y_{3}, y_{\xi_{5}}\right],
\end{aligned}
$$

For $\eta \in \Psi^{6}$, we fix the orientation by the following order;

$$
\begin{aligned}
& y_{\eta_{-7}}=y_{6}, \quad y_{\eta_{-5}}=\left[y_{5}, y_{\eta_{-7}}\right], \quad y_{\eta_{-3}}=\left[y_{4}, y_{\eta_{-5}}\right], \quad y_{\eta_{-1}}=\left[y_{3}, y_{\eta_{-3}}\right], \\
& y_{\eta_{1}}=\left[y_{2}, y_{\eta_{-3}}\right], \quad y_{\eta_{3}}=\left[y_{3}, y_{\eta_{1}}\right], \quad y_{\eta_{5}}=\left[y_{4}, y_{\eta_{3}}\right], \quad y_{\eta_{7}}=\left[y_{5}, y_{\eta_{5}}\right],
\end{aligned}
$$

Finally, for $\alpha \in \Phi_{2}^{+}$, we fix the orientation by the following;

$$
\begin{aligned}
& y_{\alpha_{-7}}=\left[y_{\eta_{-1}}, y_{\xi_{-7}}\right], \quad y_{\alpha_{-5}}=\left[y_{\eta_{3}}, y_{\xi_{-7}}\right], \quad y_{\alpha_{-3}}=\left[y_{\eta_{5}}, y_{\xi_{-7}}\right], \quad y_{\alpha_{-1}}=\left[y_{\eta_{7}}, y_{\xi_{-7}}\right] \text {, } \\
& y_{\alpha_{1}}=\left[y_{\eta_{5}}, y_{\xi_{-5}}\right], \quad y_{\alpha_{3}}=\left[y_{\eta_{7}}, y_{\xi_{-5}}\right], \quad y_{\alpha_{5}}=\left[y_{\eta_{7}}, y_{\xi_{-3}}\right], \quad y_{\alpha_{7}}=\left[y_{\eta_{7}}, y_{\xi_{1}}\right] \text {. }
\end{aligned}
$$

Then, for example, we calculate

$$
\left[y_{\eta_{-1}}, y_{\xi_{-7}}\right]=\left[\left[y_{3}, y_{\eta_{-3}}\right], y_{\xi_{-7}}\right]=\left[\left[y_{3}, y_{\xi_{-7}}\right], y_{\eta_{-3}}\right]+\left[y_{3},\left[y_{\eta_{-3}}, y_{\xi_{-7}}\right]\right]=\left[y_{\xi_{-5}}, y_{\eta_{-3}}\right] .
$$

In the same way, by the repeated application of Jacobi identities, we obtain

$$
\begin{aligned}
y_{\alpha_{-7}}=\left[y_{\eta_{-1}}, y_{\xi_{-7}}\right]=-\left[y_{\eta_{-3}}, y_{\xi_{-5}}\right]=\left[y_{\eta_{-5}}, y_{\xi_{-3}}\right]=-\left[y_{\eta_{-7}}, y_{\xi_{-1}}\right], \\
y_{\alpha_{-5}}=\left[y_{\eta_{3}}, y_{\xi_{-7}}\right]=-\left[y_{\eta_{1}}, y_{\xi_{-5}}\right]=\left[y_{\eta_{-5}}, y_{\xi_{1}}\right]=-\left[y_{\eta_{-7}}, y_{\xi_{3}}\right], \\
y_{\alpha_{-3}}=\left[y_{\eta_{5}}, y_{\xi_{-7}}\right]=-\left[y_{\eta_{1}}, y_{\xi_{-3}}\right]=\left[y_{\eta_{-3}}, y_{\xi_{1}}\right]=-\left[y_{\eta_{-7}}, y_{\xi_{5}}\right], \\
y_{\alpha_{-1}}=\left[y_{\eta_{7}}, y_{\xi_{-7}}\right]=-\left[y_{\eta_{1}}, y_{\xi_{-1}}\right]=\left[y_{\eta_{-3}}, y_{\xi_{3}}\right]=-\left[y_{\eta_{-5}}, y_{\xi_{5}}\right], \\
y_{\alpha_{1}}=\left[y_{\eta_{5}}, y_{\xi_{-5}}\right]=-\left[y_{\eta_{3}}, y_{\xi_{-3}}\right]=\left[y_{\eta_{-1}}, y_{\xi_{1}}\right]=-\left[y_{\eta_{-7}}, y_{\xi_{7}}\right], \\
y_{\alpha_{3}}=\left[y_{\eta_{7}}, y_{\xi_{-5}}\right]=-\left[y_{\eta_{3}}, y_{\xi_{-1}}\right]=\left[y_{\eta_{-1}}, y_{\xi_{3}}\right]=-\left[y_{\eta_{-5}}, y_{\xi_{7}}\right], \\
y_{\alpha_{5}}=\left[y_{\eta_{7}}, y_{\xi_{-3}}\right]=-\left[y_{\eta_{5}}, y_{\xi_{-1}}\right]=\left[y_{\eta_{-1}}, y_{\xi_{5}}\right]=-\left[y_{\eta_{-3}}, y_{\xi_{7}}\right], \\
y_{\alpha_{7}}=\left[y_{\eta_{7}}, y_{\xi_{1}}\right]=-\left[y_{\eta_{5}}, y_{\xi_{3}}\right]=\left[y_{\eta_{3}}, y_{\xi_{5}}\right]=-\left[y_{\eta_{1}}, y_{\xi_{7}}\right] .
\end{aligned}
$$

Thus, by fixing the basis $\left\{y_{\alpha_{i}}\right\}$ of $S_{-2}$ and $\left\{y_{\xi_{j}}\right\}$ of $\mathfrak{l}_{-1}$, an element $A=a_{1} \operatorname{ad}\left(y_{\eta_{-7}}\right)+$ $a_{2} \operatorname{ad}\left(y_{\eta_{-5}}\right)+a_{3} \operatorname{ad}\left(y_{\eta_{-3}}\right)+a_{4} \operatorname{ad}\left(y_{\eta_{-1}}\right)+a_{5} \operatorname{ad}\left(y_{\eta_{1}}\right)+a_{6} \operatorname{ad}\left(y_{\eta_{3}}\right)+a_{7} \operatorname{ad}\left(y_{\eta_{5}}\right)+a_{8} \operatorname{ad}\left(y_{\eta_{7}}\right)$ 
$\in S_{-1} \subset S_{-2} \otimes\left(\mathfrak{l}_{-1}\right)^{*} \cong M(8,8)$ is represented as the matrix of the following form;

$$
\left(\begin{array}{cccccccc}
a_{4} & -a_{3} & a_{2} & -a_{1} & 0 & 0 & 0 & 0 \\
a_{6} & -a_{5} & 0 & 0 & a_{2} & -a_{1} & 0 & 0 \\
a_{7} & 0 & -a_{5} & 0 & a_{3} & 0 & -a_{1} & 0 \\
a_{8} & 0 & 0 & -a_{5} & 0 & a_{3} & -a_{2} & 0 \\
0 & a_{7} & -a_{6} & 0 & a_{4} & 0 & 0 & -a_{1} \\
0 & a_{8} & 0 & -a_{6} & 0 & a_{4} & 0 & -a_{2} \\
0 & 0 & a_{8} & -a_{7} & 0 & 0 & a_{4} & -a_{3} \\
0 & 0 & 0 & 0 & a_{8} & -a_{7} & a_{6} & -a_{5}
\end{array}\right)
$$

Hence the standard differential system $\left(M(\mathfrak{m}), D_{\mathfrak{m}}\right)$ of type $\mathfrak{m}$ in this case is given by

$$
D_{\mathfrak{m}}=\left\{\varpi_{1}=\varpi_{2}=\cdots=\varpi_{8}=0\right\},
$$

where

$$
\begin{array}{ll}
\varpi_{1}=d y_{1}+p_{4} d x_{1}-p_{3} d x_{2}+p_{2} d x_{3}-p_{1} d x_{4}, & \varpi_{2}=d y_{2}+p_{6} d x_{1}-p_{5} d x_{2}+p_{2} d x_{5}-p_{1} d x_{6}, \\
\varpi_{3}=d y_{3}+p_{7} d x_{1}-p_{5} d x_{3}+p_{3} d x_{5}-p_{1} d x_{7}, & \varpi_{4}=d y_{4}+p_{8} d x_{1}-p_{5} d x_{4}+p_{3} d x_{6}-p_{2} d x_{7}, \\
\varpi_{5}=d y_{5}+p_{7} d x_{2}-p_{6} d x_{3}+p_{4} d x_{5}-p_{1} d x_{8}, & \varpi_{6}=d y_{6}+p_{8} d x_{2}-p_{6} d x_{4}+p_{4} d x_{6}-p_{2} d x_{8}, \\
\varpi_{7}=d y_{7}+p_{8} d x_{3}-p_{7} d x_{4}+p_{4} d x_{7}-p_{3} d x_{8}, & \varpi_{8}=d y_{8}+p_{8} d x_{5}-p_{7} d x_{6}+p_{6} d x_{7}-p_{5} d x_{8} .
\end{array}
$$

Here $\left(y_{1}, \ldots, y_{8}, x_{1}, \ldots, x_{8}, p_{1}, \ldots, p_{8}\right)$ is a coordinate system of $M(\mathfrak{m}) \cong \mathbb{K}^{24}$. Thus the model equation of our typical symbol $\mathfrak{m}=\check{\mathfrak{g}}_{-2} \oplus \check{\mathfrak{g}}_{-1} \subset \mathfrak{C}^{1}\left(\mathfrak{l}_{-1}, S_{-2}\right)$ is given by

$$
\begin{array}{lll}
\frac{\partial y_{1}}{\partial x_{4}}=\frac{\partial y_{2}}{\partial x_{6}}=\frac{\partial y_{3}}{\partial x_{7}}=\frac{\partial y_{5}}{\partial x_{8}}, & \frac{\partial y_{1}}{\partial x_{3}}=\frac{\partial y_{2}}{\partial x_{5}}=-\frac{\partial y_{4}}{\partial x_{7}}=-\frac{\partial y_{6}}{\partial x_{8}} \\
\frac{\partial y_{1}}{\partial x_{2}}=-\frac{\partial y_{3}}{\partial x_{5}}=-\frac{\partial y_{4}}{\partial x_{6}}=\frac{\partial y_{7}}{\partial x_{8}}, & \frac{\partial y_{1}}{\partial x_{1}}=\frac{\partial y_{5}}{\partial x_{5}}=\frac{\partial y_{6}}{\partial x_{6}}=\frac{\partial y_{7}}{\partial x_{7}} \\
\frac{\partial y_{2}}{\partial x_{2}}=\frac{\partial y_{3}}{\partial x_{3}}=\frac{\partial y_{4}}{\partial x_{4}}=\frac{\partial y_{8}}{\partial x_{8}}, & \frac{\partial y_{2}}{\partial x_{1}}=-\frac{\partial y_{5}}{\partial x_{3}}=-\frac{\partial y_{6}}{\partial x_{4}}=\frac{\partial y_{8}}{\partial x_{7}} \\
\frac{\partial y_{3}}{\partial x_{1}}=\frac{\partial y_{5}}{\partial x_{2}}=-\frac{\partial y_{7}}{\partial x_{4}}=-\frac{\partial y_{8}}{\partial x_{6}}, & \frac{\partial y_{4}}{\partial x_{1}}=\frac{\partial y_{6}}{\partial x_{2}}=\frac{\partial y_{7}}{\partial x_{3}}=\frac{\partial y_{8}}{\partial x_{5}} \\
\frac{\partial y_{i}}{\partial x_{j}}=0 & \text { otherwise }
\end{array}
$$

where $y_{1}, \ldots, y_{8}$ are dependent variables and $x_{1}, \ldots, x_{8}$ are independent variables. By a direct calculation, we see that the prolongation of the first order system (4.2) is given by

$$
\frac{\partial^{2} y_{i}}{\partial x_{j} \partial x_{k}}=0 \quad \text { for } \quad 1 \leqq i, j, k \leqq 8 .
$$

Case $(2)\left[\left(D_{5},\left\{\alpha_{5}\right\}\right), \varpi_{5},\left(E_{6},\left\{\gamma_{1}, \gamma_{3}\right\}\right)\right]$.

For the gradation of type $\left(E_{6},\left\{\gamma_{1}, \gamma_{3}\right\}\right)$, we have

$$
\begin{aligned}
& \Phi_{3}^{+}=\left\{\alpha_{1}=12 \underset{1}{2} 10, \alpha_{2}=12 \underset{1}{2} 11, \alpha_{3}=12 \underset{1}{2} 1, \alpha_{4}=12 \underset{1}{3} 21, \alpha_{5}=12 \underset{2}{3} 21\right\} \\
& \Phi_{2}^{+}=\left\{\eta_{1}=1 \underset{0}{1000}, \quad \eta_{2}=11 \underset{0}{1} 00, \quad \eta_{3}=11 \underset{1}{100}, \quad \eta_{4}=11 \underset{0}{110}, \quad \eta_{5}=11{ }_{1}^{110},\right.
\end{aligned}
$$

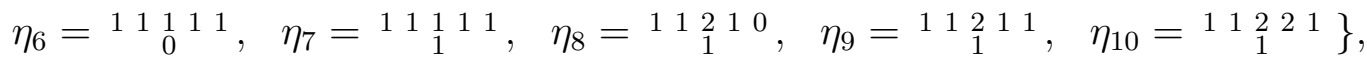




$$
\begin{aligned}
& \Phi_{1}^{+}=\Psi^{1} \cup \Psi^{3} \\
& \Psi^{3}=\left\{\xi_{1}=01 \underset{0}{0} 00, \quad \xi_{2}=01 \underset{0}{1} 00, \quad \xi_{3}=01 \underset{1}{100}, \quad \xi_{4}=01 \underset{0}{1} 100, \quad \xi_{5}=01{ }_{1}^{110},\right. \\
& \left.\xi_{6}=01{ }_{0}^{11} 11, \quad \xi_{7}=01 \underset{1}{1} 11, \quad \xi_{8}=01 \underset{1}{210}, \quad \xi_{9}=01 \underset{1}{2} 11, \quad \xi_{10}=01 \underset{1}{2} 1\right\} \text {, } \\
& \Psi^{1}=\left\{\gamma_{1}=\begin{array}{lllll}
1 & 0 & 0 & 0 & 0 \\
0 & 0
\end{array}\right\}
\end{aligned}
$$

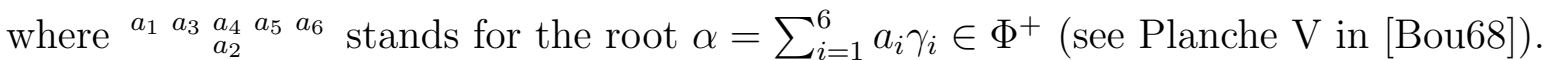

Thus we have $\mu=3$,

$$
\mathfrak{m}=\check{\mathfrak{g}}_{-3} \oplus \check{\mathfrak{g}}_{-2} \oplus \check{\mathfrak{g}}_{-1} \quad \text { and } \quad \check{\mathfrak{g}}_{-1}=S_{-1} \oplus \mathfrak{l}_{-1},
$$

where $\check{\mathfrak{g}}_{-3}=S_{-3}, \check{\mathfrak{g}}_{-2}=S_{-2}, S_{-1}$ and $\mathfrak{l}_{-1}$ are spanned by the root spaces $\mathfrak{g}_{-\beta}$ for $\beta \in$ $\Phi_{3}^{+}, \Phi_{2}^{+}, \Psi^{1}$ and $\Psi^{3}$ respectively. Hence $\operatorname{dim} S_{-3}=5, \operatorname{dim} S_{-2}=\operatorname{dim} \mathfrak{l}_{-1}=10$ and $\operatorname{dim} S_{-1}=1$.

For $\Phi_{3}^{+}, \Phi_{2}^{+}, \Psi^{1}$ and $\Psi^{3}$, we observe that $\alpha+\beta \notin \Phi$ for $\alpha, \beta \in \Phi_{3}^{+} \cup \Phi_{2}^{+} \cup \Psi^{1}, \xi-\gamma \notin \Phi$ for $\xi \in \Psi^{3}, \gamma \in \Psi^{1}$ and that, if $\eta+\xi \in \Phi_{3}^{+}$for $\eta \in \Phi_{2}^{+}, \xi \in \Psi^{3}$, then $\eta-\xi \notin \Phi$. This implies that $\left[y_{\alpha}, y_{\beta}\right]=0$ for $\alpha, \beta \in \Phi_{3}^{+} \cup \Phi_{2}^{+} \cup \Psi^{1},\left[y_{\gamma}, y_{\xi}\right]= \pm y_{\gamma+\xi}$ for $\xi \in \Psi^{3}, \gamma \in \Psi^{1}$, if $\gamma+\xi \in \Phi$ and $\left[y_{\eta}, y_{\xi}\right]= \pm y_{\eta+\xi}$ for $\eta \in \Phi_{2}^{+}, \xi \in \Psi^{3}$, if $\eta+\xi \in \Phi$, by the property of the Chevalley basis. Hence, from Planche $\mathrm{V}$ in [Bou68], we readily obtain the non-trivial bracket relation among $\check{\mathfrak{g}}_{-1}$ and $\left[\check{\mathfrak{g}}_{-2}, \mathfrak{l}_{-1}\right]$ as in (4.4) and (4.5) below up to signs.

We fix the signs of $y_{\beta}$ for $\beta \in \Phi_{3}^{+}, \Phi_{2}^{+}, \Psi^{3}$ and $\Psi^{1}$ as follows: First we choose the orientation of $y_{\gamma_{i}}$ for simple roots by fixing the root vectors $y_{i}=y_{\gamma_{i}} \in \mathfrak{g}_{-\gamma_{i}}$. For $\xi \in \Psi^{3}$, we fix the orientation by the following order;

$$
\begin{aligned}
& y_{\xi_{1}}=y_{3}, \quad y_{\xi_{2}}=\left[y_{4}, y_{\xi_{1}}\right], \quad y_{\xi_{3}}=\left[y_{2}, y_{\xi_{2}}\right], \quad y_{\xi_{4}}=\left[y_{5}, y_{\xi_{2}}\right], \quad y_{\xi_{5}}=\left[y_{5}, y_{\xi_{3}}\right], \\
& y_{\xi_{6}}=\left[y_{6}, y_{\xi_{4}}\right], \quad y_{\xi_{7}}=\left[y_{2}, y_{\xi_{6}}\right], \quad y_{\xi_{8}}=\left[y_{4}, y_{\xi_{5}}\right], \quad y_{\xi_{9}}=\left[y_{6}, y_{\xi_{8}}\right], \quad y_{\xi_{10}}=\left[y_{5}, y_{\xi_{9}}\right] \text {. }
\end{aligned}
$$

For $\eta \in \Phi_{2}^{+}$, we fix the orientation by the following ;

$$
y_{\eta_{i}}=\left[y_{1}, y_{\xi_{i}}\right] \quad \text { for } \quad i=1,2, \ldots, 10 .
$$

Finally, for $\alpha \in \Phi_{3}^{+}$, we fix the orientation by the following;

$$
y_{\alpha_{1}}=\left[y_{\eta_{8}}, y_{\xi_{1}}\right], \quad y_{\alpha_{2}}=\left[y_{\eta_{9}}, y_{\xi_{1}}\right], \quad y_{\alpha_{3}}=\left[y_{\eta_{10}}, y_{\xi_{1}}\right], \quad y_{\alpha_{4}}=\left[y_{\eta_{10}}, y_{\xi_{2}}\right], \quad y_{\alpha_{5}}=\left[y_{\eta_{10}}, y_{\xi_{3}}\right] .
$$

Then, for example, we calculate

$$
\left[y_{\eta_{p}}, y_{\xi_{q}}\right]=\left[\left[y_{1}, y_{\xi_{p}}\right], y_{\xi_{q}}\right]=\left[\left[y_{1}, y_{\xi_{q}}\right], y_{\xi_{p}}\right]=\left[y_{\eta_{q}}, y_{\xi_{p}}\right] \quad \text { for } \quad 1 \leqq p, q \leqq 10
$$

and

$$
\begin{aligned}
{\left[y_{\eta_{5}}, y_{\xi_{2}}\right]=\left[y_{\eta_{5}},\left[y_{4}, y_{\xi_{1}}\right]\right]=\left[\left[y_{\eta_{5}}, y_{4}\right], y_{\xi_{1}}\right] } & =\left[\left[\left[y_{1}, y_{\xi_{5}}\right], y_{4}\right], y_{\xi_{1}}\right] \\
& =\left[\left[y_{1},\left[y_{\xi_{5}}, y_{4}\right]\right], y_{\xi_{1}}\right]=-\left[\left[y_{1}, y_{\xi_{8}}\right], y_{\xi_{1}}\right]=-\left[y_{\eta_{8}}, y_{\xi_{1}}\right] .
\end{aligned}
$$

In the same way, by the repeated application of Jacobi identities, we obtain

$$
\begin{aligned}
& y_{\alpha_{1}}=\left[y_{\eta_{8}}, y_{\xi_{1}}\right]=-\left[y_{\eta_{5}}, y_{\xi_{2}}\right]=\left[y_{\eta_{4}}, y_{\xi_{3}}\right]=\left[y_{\eta_{3}}, y_{\xi_{4}}\right]=-\left[y_{\eta_{2}}, y_{\xi_{5}}\right]=\left[y_{\eta_{1}}, y_{\xi_{8}}\right], \\
& y_{\alpha_{2}}=\left[y_{\eta_{9}}, y_{\xi_{1}}\right]=-\left[y_{\eta_{7}}, y_{\xi_{2}}\right]=\left[y_{\eta_{6}}, y_{\xi_{3}}\right]=\left[y_{\eta_{3}}, y_{\xi_{6}}\right]=-\left[y_{\eta_{2}}, y_{\xi_{7}}\right]=\left[y_{\eta_{1}}, y_{\xi_{9}}\right], \\
& y_{\alpha_{3}}=\left[y_{\eta_{10}}, y_{\xi_{1}}\right]=-\left[y_{\eta_{7}}, y_{\xi_{4}}\right]=\left[y_{\eta_{6}}, y_{\xi_{5}}\right]=\left[y_{\eta_{5}}, y_{\xi_{6}}\right]=-\left[y_{\eta_{4}}, y_{\xi_{7}}\right]=\left[y_{\eta_{1}}, y_{\xi_{10}}\right], \\
& y_{\alpha_{4}}=\left[y_{\eta_{10}}, y_{\xi_{2}}\right]=-\left[y_{\eta_{9}}, y_{\xi_{4}}\right]=\left[y_{\eta_{8}}, y_{\xi_{6}}\right]=\left[y_{\eta_{6}}, y_{\xi_{8}}\right]=-\left[y_{\eta_{4}}, y_{\xi_{9}}\right]=\left[y_{\eta_{2}}, y_{\xi_{10}}\right], \\
& y_{\alpha_{5}}=\left[y_{\eta_{10}}, y_{\xi_{3}}\right]=-\left[y_{\eta_{9}}, y_{\xi_{5}}\right]=\left[y_{\eta_{8}}, y_{\xi_{7}}\right]=\left[y_{\eta_{7}}, y_{\xi_{8}}\right]=-\left[y_{\eta_{5}}, y_{\xi_{9}}\right]=\left[y_{\eta_{3}}, y_{\xi_{10}}\right] .
\end{aligned}
$$


Then, by fixing the basis $\left\{y_{\alpha_{i}}\right\}_{i=1}^{5}$ of $S_{-3}$ and $\left\{y_{\xi_{j}}\right\}_{j=1}^{10}$ of $\mathfrak{l}_{-1}$, an element $A=\sum_{j=1}^{10} a_{j} \operatorname{ad}\left(y_{\eta_{j}}\right)$ $\in S_{-2} \subset S_{-3} \otimes\left(\mathfrak{l}_{-1}\right)^{*} \cong M(5,10)$ is represented as the matrix of the following form;

$$
\left(\begin{array}{cccccccccc}
a_{8} & -a_{5} & a_{4} & a_{3} & -a_{2} & 0 & 0 & a_{1} & 0 & 0 \\
a_{9} & -a_{7} & a_{6} & 0 & 0 & a_{3} & -a_{2} & 0 & a_{1} & 0 \\
a_{10} & 0 & 0 & -a_{7} & a_{6} & a_{5} & -a_{4} & 0 & 0 & a_{1} \\
0 & a_{10} & 0 & -a_{9} & 0 & a_{8} & 0 & a_{6} & -a_{4} & a_{2} \\
0 & 0 & a_{10} & 0 & -a_{9} & 0 & a_{8} & a_{7} & -a_{5} & a_{3}
\end{array}\right)
$$

Moreover, for $y_{1} \in S_{-1}$, we have

$$
\begin{aligned}
& y_{\alpha_{1}}=\left[\left[y_{1}, y_{\xi_{1}}\right], y_{\xi_{8}}\right]=-\left[\left[y_{1}, y_{\xi_{2}}\right], y_{\xi_{5}}\right]=\left[\left[y_{1}, y_{\xi_{3}}\right], y_{\xi_{4}}\right], \\
& y_{\alpha_{2}}=\left[\left[y_{1}, y_{\xi_{1}}\right], y_{\xi_{9}}\right]=-\left[\left[y_{1}, y_{\xi_{2}}\right], y_{\xi_{7}}\right]=\left[\left[y_{1}, y_{\xi_{3}}\right], y_{\xi_{6}}\right], \\
& y_{\alpha_{3}}=\left[\left[y_{1}, y_{\xi_{1}}\right], y_{\xi_{10}}\right]=-\left[\left[y_{1}, y_{\xi_{4}}\right], y_{\xi_{7}}\right]=\left[\left[y_{1}, y_{\xi_{5}}\right], y_{\xi_{6}}\right], \\
& y_{\alpha_{4}}=\left[\left[y_{1}, y_{\xi_{2}}\right], y_{\xi_{10}}\right]=-\left[\left[y_{1}, y_{\xi_{4}}\right], y_{\xi_{9}}\right]=\left[\left[y_{1}, y_{\xi_{6}}\right], y_{\xi_{8}}\right], \\
& y_{\alpha_{5}}=\left[\left[y_{1}, y_{\xi_{3}}\right], y_{\xi_{10}}\right]=-\left[\left[y_{1}, y_{\xi_{5}}\right], y_{\xi_{9}}\right]=\left[\left[y_{1}, y_{\xi_{7}}\right], y_{\xi_{8}}\right] .
\end{aligned}
$$

Thus $S_{-1}$ is embedded as the 1-dimensional subspace of $S_{-3} \otimes S^{2}\left(\left(\mathfrak{l}_{-1}\right)^{*}\right)$ spanned by the following quadratic form $f$

$$
\begin{array}{r}
f(X, X)=\left(x_{1} x_{8}-x_{2} x_{5}+x_{3} x_{4}\right) y_{\alpha_{1}}+\left(x_{1} x_{9}-x_{2} x_{7}+x_{3} x_{6}\right) y_{\alpha_{2}}+\left(x_{1} x_{10}-x_{4} x_{7}+x_{5} x_{6}\right) y_{\alpha_{3}} \\
+\left(x_{2} x_{10}-x_{4} x_{9}+x_{6} x_{8}\right) y_{\alpha_{4}}+\left(x_{3} x_{10}-x_{5} x_{9}+x_{7} x_{8}\right) y_{\alpha_{5}} .
\end{array}
$$

for $X=\sum_{j=1}^{10} x_{j} y_{\xi_{j}} \in \mathfrak{l}_{-1}$.

By the above matrix representation, we can embed $S_{-2}$ as a subspace of $S_{-3} \otimes\left(\mathfrak{l}_{-1}\right)^{*} \cong$ $M(5,10)$ and obtain the following first order system as the model equation whose symbol coincides with this subspace:

$$
\begin{aligned}
\frac{\partial y_{1}}{\partial x_{8}} & =\frac{\partial y_{2}}{\partial x_{9}}=\frac{\partial y_{3}}{\partial x_{10}}, & -\frac{\partial y_{1}}{\partial x_{5}} & =-\frac{\partial y_{2}}{\partial x_{7}}=\frac{\partial y_{4}}{\partial x_{10}}, & & \frac{\partial y_{1}}{\partial x_{4}}=\frac{\partial y_{2}}{\partial x_{6}}=\frac{\partial y_{5}}{\partial x_{10}}, \\
\frac{\partial y_{1}}{\partial x_{3}} & =-\frac{\partial y_{3}}{\partial x_{7}}=-\frac{\partial y_{4}}{\partial x_{9}}, & -\frac{\partial y_{1}}{\partial x_{2}} & =\frac{\partial y_{3}}{\partial x_{6}}=-\frac{\partial y_{5}}{\partial x_{9}}, & & \frac{\partial y_{2}}{\partial x_{3}}=\frac{\partial y_{3}}{\partial x_{5}}=\frac{\partial y_{4}}{\partial x_{8}} \\
-\frac{\partial y_{3}}{\partial x_{4}} & =\frac{\partial y_{5}}{\partial x_{8}}, & \frac{\partial y_{1}}{\partial x_{1}} & =\frac{\partial y_{4}}{\partial x_{6}}=\frac{\partial y_{5}}{\partial x_{7}}, & \frac{\partial y_{2}}{\partial x_{1}} & =-\frac{\partial y_{4}}{\partial x_{4}}=-\frac{\partial y_{5}}{\partial x_{5}}, \\
\frac{\partial y_{4}}{\partial x_{2}} & =\frac{\partial y_{5}}{\partial x_{3}}, & \frac{\partial y_{i}}{\partial x_{j}} & =0 & \text { otherwise, } &
\end{aligned}
$$

where $y_{1}, \ldots, y_{5}$ are dependent variables and $x_{1}, \ldots, x_{10}$ are independent variables. Moreover, by a direct calculation, we see that the prolongation of the first order system (4.6) is given by

$$
\begin{aligned}
& \frac{\partial^{2} y_{1}}{\partial x_{1} \partial x_{8}}=\frac{\partial^{2} y_{2}}{\partial x_{1} \partial x_{9}}=\frac{\partial^{2} y_{3}}{\partial x_{1} \partial x_{10}}=\frac{\partial^{2} y_{4}}{\partial x_{10} \partial x_{2}}=\frac{\partial^{2} y_{5}}{\partial x_{10} \partial x_{3}} \\
&=\frac{\partial^{2} y_{2}}{\partial x_{3} \partial x_{6}}=\frac{\partial^{2} y_{1}}{\partial x_{3} \partial x_{4}}=-\frac{\partial^{2} y_{3}}{\partial x_{4} \partial x_{7}}=-\frac{\partial^{2} y_{4}}{\partial x_{4} \partial x_{9}}=-\frac{\partial^{2} y_{5}}{\partial x_{9} \partial x_{5}} \\
&=\frac{\partial^{2} y_{3}}{\partial x_{5} \partial x_{6}}=-\frac{\partial^{2} y_{1}}{\partial x_{5} \partial x_{2}}=-\frac{\partial^{2} y_{2}}{\partial x_{2} \partial x_{7}}=\frac{\partial^{2} y_{5}}{\partial x_{7} \partial x_{8}}=\frac{\partial^{2} y_{4}}{\partial x_{8} \partial x_{6}}, \\
& \frac{\partial^{2} y_{i}}{\partial x_{p} \partial x_{q}}=0 \quad \text { otherwise. }
\end{aligned}
$$


From the above expression of $f$, we observe that the above second order system is the model equation of the 1-dimensional embedded subspace $S_{-1}$ in $S_{-3} \otimes S^{2}\left(\left(\mathfrak{l}_{-1}\right)^{*}\right)$.Furthermore, by a direct calculation, we see that the prolongation of this second order system (4.7) is given by

$$
\frac{\partial^{3} y_{i}}{\partial x_{p} \partial x_{q} \partial x_{r}}=0 \quad \text { for } \quad 1 \leqq i \leqq 5, \quad 1 \leqq p, q, r \leqq 10
$$

Case $(3)\left[\left(D_{5},\left\{\alpha_{4}\right\}\right), \varpi_{5},\left(E_{6},\left\{\gamma_{1}, \gamma_{2}\right\}\right)\right]$.

For the gradation of type $\left(E_{6},\left\{\gamma_{1}, \gamma_{2}\right\}\right)$, we have

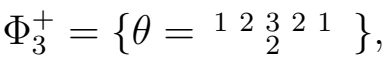

$$
\begin{aligned}
& \Phi_{2}^{+}=\left\{\eta_{1}=12 \underset{1}{3} 21, \quad \eta_{2}=12 \underset{1}{2} 21, \quad \eta_{3}=11 \underset{1}{2} 21, \quad \eta_{4}=12 \underset{1}{2} 11, \quad \eta_{5}=1{ }_{1}^{2} 11,\right.
\end{aligned}
$$

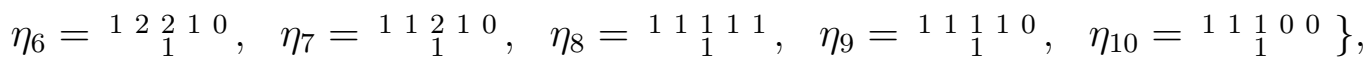

$$
\begin{aligned}
& \Phi_{1}^{+}=\Psi^{2} \cup \Psi^{1}
\end{aligned}
$$

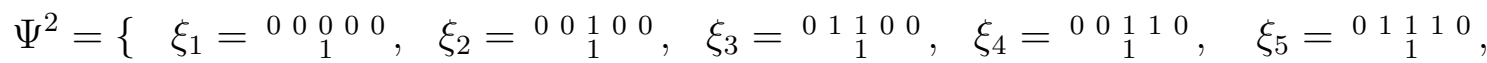

$$
\begin{aligned}
& \left.\xi_{6}=00{ }_{1}^{1} 11, \quad \xi_{7}=01{ }_{1}^{1} 11, \quad \xi_{8}=01 \underset{1}{2} 10, \quad \xi_{9}=01 \underset{1}{2} 11, \quad \xi_{10}=01{ }_{1}^{2} 21\right\} \text {, }
\end{aligned}
$$

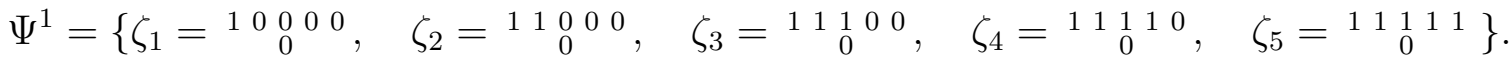

where $\begin{array}{ccccc}a_{1} & a_{3} & a_{4} & a_{5} & a_{6} \\ & & a_{2} & & \\ & & \end{array}$ stands for the root $\alpha=\sum_{i=1}^{6} a_{i} \gamma_{i} \in \Phi^{+}$(see Planche V in [Bou68]).

Thus we have $\mu=3$,

$$
\mathfrak{m}=\check{\mathfrak{g}}_{-3} \oplus \check{\mathfrak{g}}_{-2} \oplus \check{\mathfrak{g}}_{-1} \quad \text { and } \quad \check{\mathfrak{g}}_{-1}=S_{-1} \oplus \mathfrak{l}_{-1},
$$

where $\check{\mathfrak{g}}_{-3}=S_{-3}, \check{\mathfrak{g}}_{-2}=S_{-2}, S_{-1}$ and $\mathfrak{l}_{-1}$ are spanned by the root spaces $\mathfrak{g}_{-\beta}$ for $\beta \in \Phi_{3}^{+}, \Phi_{2}^{+}, \Psi^{1}$ and $\Psi^{2}$ respectively. Hence $\operatorname{dim} S_{-3}=1, \operatorname{dim} S_{-2}=\operatorname{dim} \mathfrak{l}_{-1}=10$ and $\operatorname{dim} S_{-1}=5$.

For $\Phi_{3}^{+}, \Phi_{2}^{+}, \Psi^{1}$ and $\Psi^{2}$, we observe that $\Phi_{3}^{+}=\{\theta\}$, where $\theta$ is the highest root, $\alpha+\beta \notin \Phi$ for $\alpha, \beta \in \Phi_{3}^{+} \cup \Phi_{2}^{+} \cup \Psi^{1}, \zeta-\xi \notin \Phi$ for $\xi \in \Psi^{2}, \zeta \in \Psi^{1}$ and that $\eta_{i}+\xi_{i}=\theta, \eta_{i}-\xi_{i} \notin \Phi$, $\xi_{i}+\xi_{j} \notin \Phi$ and $\eta_{i}+\xi_{j} \notin \Phi$ if $i \neq j$ for $\eta_{i} \in \Phi_{2}^{+}$and $\xi_{i}, \xi_{j} \in \Psi^{2}(i, j=1, \ldots, 10)$. This implies that $\left[y_{\alpha}, y_{\beta}\right]=0$ for $\alpha, \beta \in \Phi_{3}^{+} \cup \Phi_{2}^{+} \cup \Psi^{1},\left[y_{\zeta}, y_{\xi}\right]= \pm y_{\zeta+\xi}$ for $\xi \in \Psi^{2}, \zeta \in \Psi^{1}$, if $\zeta+\xi \in \Phi$ and that $\left[y_{\xi_{i}}, y_{\xi_{j}}\right]=0,\left[y_{\eta_{i}}, y_{\xi_{j}}\right]= \pm \delta_{i j} y_{\theta}$ for $\eta_{i} \in \Phi_{2}^{+}, \xi_{i}, \xi_{j} \in \Psi^{2}(i, j=1, \ldots, 10)$, by the property of the Chevalley basis. Hence, from Planche $\mathrm{V}$ in [Bou68], we readily obtain the non-trivial bracket relation among $\check{\mathfrak{g}}_{-1}$ and $\left[\check{\mathfrak{g}}_{-2}, \mathfrak{l}_{-1}\right]$ as in (4.9) and (4.10) below up to signs.

We fix the signs of $y_{\beta}$ for $\beta \in \Phi_{3}^{+}, \Phi_{2}^{+}, \Psi^{2}$ and $\Psi^{1}$ as follows: First we choose the orientation of $y_{\gamma_{i}}$ for simple roots by fixing the root vectors $y_{i}=y_{\gamma_{i}} \in \mathfrak{g}_{-\gamma_{i}}$. For $\zeta \in \Psi^{1}$, we fix the orientation by the following order;

$$
y_{\zeta_{1}}=y_{1}, \quad y_{\zeta_{2}}=\left[y_{3}, y_{\zeta_{1}}\right], \quad y_{\zeta_{3}}=\left[y_{4}, y_{\zeta_{2}}\right], \quad y_{\zeta_{4}}=\left[y_{5}, y_{\zeta_{3}}\right], \quad y_{\zeta_{5}}=\left[y_{6}, y_{\zeta_{4}}\right] .
$$

For $\xi \in \Psi^{2}$, we fix the orientation by the following order;

$$
\begin{aligned}
& y_{\xi_{1}}=y_{2}, \quad y_{\xi_{2}}=\left[y_{\xi_{1}}, y_{4}\right], \quad y_{\xi_{3}}=\left[y_{\xi_{2}}, y_{3}\right], \quad y_{\xi_{4}}=\left[y_{\xi_{2}}, y_{5}\right], \quad y_{\xi_{5}}=\left[y_{\xi_{3}}, y_{5}\right] \\
& y_{\xi_{6}}=\left[y_{\xi_{4}}, y_{6}\right], \quad y_{\xi_{7}}=\left[y_{\xi_{5}}, y_{6}\right], \quad y_{\xi_{8}}=\left[y_{\xi_{5}}, y_{4}\right], \quad y_{\xi_{9}}=\left[y_{\xi_{8}}, y_{6}\right], \quad y_{\xi_{10}}=\left[y_{\xi_{9}}, y_{5}\right] \text {. }
\end{aligned}
$$

For $\eta \in \Phi_{2}^{+}$, we fix the orientation by the following order;

$$
\begin{aligned}
& y_{\eta_{1}}=\left[y_{\zeta_{3}}, y_{\xi_{10}}\right], \quad y_{\eta_{2}}=\left[y_{\zeta_{2}}, y_{\xi_{10}}\right], \quad y_{\eta_{3}}=\left[y_{\zeta_{1}}, y_{\xi_{10}}\right], \quad y_{\eta_{4}}=-\left[y_{\zeta_{2}}, y_{\xi_{9}}\right], \\
& y_{\eta_{5}}=-\left[y_{\zeta_{1}}, y_{\xi_{9}}\right], \quad y_{\eta_{6}}=\left[y_{\zeta_{2}}, y_{\xi_{8}}\right], \quad y_{\eta_{7}}=\left[y_{\zeta_{1}}, y_{\xi_{8}}\right], \quad y_{\eta_{8}}=\left[y_{\zeta_{1}}, y_{\xi_{7}}\right], \\
& y_{\eta_{9}}=-\left[y_{\zeta_{1}}, y_{\xi_{5}}\right], \quad y_{\eta_{10}}=\left[y_{\zeta_{1}}, y_{\xi_{3}}\right] \text {. }
\end{aligned}
$$


Finally, for $\theta \in \Phi_{3}^{+}$, we fix the orientation by the following;

$$
y_{\theta}=\left[y_{\eta_{10}}, y_{\xi_{10}}\right]
$$

Then, for example, we calculate

$$
\begin{aligned}
{\left[y_{\eta_{10}}, y_{\xi_{10}}\right]=\left[y_{\eta_{10}},\left[y_{\xi_{9}}, y_{5}\right]\right] } & =\left[y_{\xi_{9}},\left[y_{\eta_{10}}, y_{5}\right]\right]=\left[y_{\xi_{9}},\left[\left[y_{\zeta_{1}}, y_{\xi_{3}}\right], y_{5}\right]\right] \\
= & {\left[y_{\xi_{9}},\left[y_{\zeta_{1}},\left[y_{\xi_{3}}, y_{5}\right]\right]\right]=\left[y_{\xi_{9}},\left[y_{\zeta_{1}}, y_{\xi_{5}}\right]\right]=\left[y_{\xi_{9}},-y_{\eta_{9}}\right]=\left[y_{\eta_{9}}, y_{\xi_{9}}\right], }
\end{aligned}
$$

and obtain

$$
\left[y_{\eta_{i}}, y_{\xi_{j}}\right]=\delta_{i j} y_{\theta}, \quad\left[y_{\eta_{i}} \cdot y_{\eta_{j}}\right]=\left[y_{\xi_{i}}, y_{\xi_{j}}\right]=0 \quad \text { for } \quad 1 \leqq i, j \leqq 10 .
$$

Moreover we calculate as in

$$
\begin{array}{r}
{\left[y_{\zeta_{3}}, y_{\xi_{7}}\right]=\left[\left[y_{4}, y_{\zeta_{2}}\right], y_{\xi_{7}}\right]=\left[\left[y_{4}, y_{\xi_{7}}\right], y_{\zeta_{2}}\right]=\left[\left[y_{4},\left[y_{\xi_{5}}, y_{6}\right]\right], y_{\zeta_{2}}\right]} \\
\left.=\left[\left[y_{4}, y_{\xi_{5}}\right], y_{6}\right], y_{\zeta_{2}}\right]=\left[\left[-y_{\xi_{8}}, y_{6}\right], y_{\zeta_{2}}\right]=-\left[y_{\xi_{9}}, y_{\zeta_{2}}\right]=-y_{\eta_{4}},
\end{array}
$$

and obtain

$$
\begin{aligned}
& y_{\theta}=\left[\left[y_{\zeta_{1}}, y_{\xi_{3}}\right], y_{\xi_{10}}\right]=-\left[\left[y_{\zeta_{1}}, y_{\xi_{5}}\right], y_{\xi_{9}}\right]=\left[\left[y_{\zeta_{1}}, y_{\xi_{7}}\right], y_{\xi_{8}}\right], \\
& y_{\theta}=\left[\left[y_{\zeta_{2}}, y_{\xi_{2}}\right], y_{\xi_{10}}\right]=-\left[\left[y_{\zeta_{2}}, y_{\xi_{4}}\right], y_{\xi_{9}}\right]=\left[\left[y_{\zeta_{2}}, y_{\xi_{6}}\right], y_{\xi_{8}}\right], \\
& y_{\theta}=\left[\left[y_{\zeta_{3}}, y_{\xi_{1}}\right], y_{\xi_{10}}\right]=-\left[\left[y_{\zeta_{3}}, y_{\xi_{4}}\right], y_{\xi_{7}}\right]=\left[\left[y_{\zeta_{3}}, y_{\xi_{5}}\right], y_{\xi_{6}}\right], \\
& y_{\theta}=\left[\left[y_{\zeta_{4}}, y_{\xi_{1}}\right], y_{\xi_{9}}\right]=-\left[\left[y_{\zeta_{4}}, y_{\xi_{2}}\right], y_{\xi_{7}}\right]=\left[\left[y_{\zeta_{4}}, y_{\xi_{3}}\right], y_{\xi_{6}}\right], \\
& y_{\theta}=\left[\left[y_{\zeta_{5}}, y_{\xi_{1}}\right], y_{\xi_{8}}\right]=-\left[\left[y_{\zeta_{2}}, y_{\xi_{2}}\right], y_{\xi_{5}}\right]=\left[\left[y_{\zeta_{5}}, y_{\xi_{3}}\right], y_{\xi_{4}}\right] .
\end{aligned}
$$

From (4.9), we have $S_{-2}=V^{*}$, by fixing the base of $S_{-3} \cong \mathbb{K}$ and putting $\mathfrak{l}_{-1}=V$. Moreover, from (4.10), $S_{-1}$ is embedded as the 5-dimensional subspace of $S^{2}\left(V^{*}\right)$ spanned by the following quadratic forms $f_{1}, \ldots, f_{5}$;

$$
\begin{array}{ll}
f_{1}(X, X)=x_{3} x_{10}-x_{5} x_{9}+x_{7} x_{8}, & f_{2}(X, X)=x_{2} x_{10}-x_{4} x_{9}+x_{6} x_{8}, \\
f_{3}(X, X)=x_{1} x_{10}-x_{4} x_{7}+x_{5} x_{6}, & f_{4}(X, X)=x_{1} x_{9}-x_{2} x_{7}+x_{3} x_{6}, \\
f_{5}(X, X)=x_{1} x_{8}-x_{2} x_{5}+x_{3} x_{4}, &
\end{array}
$$

for $X=\sum_{i=1}^{10} x_{i} y_{\xi_{i}} \in \mathfrak{l}_{-1}$. Thus, by fixing the basis $\left\{y_{\theta}\right\}$ of $S_{-3}$ and $\left\{y_{\xi_{1}}, \ldots, y_{\xi_{10}}\right\}$ of $\mathfrak{l}_{-1}$, an element $A=\sum_{i=1}^{5} a_{i} \operatorname{ad}\left(y_{\zeta_{i}}\right) \in S_{-1} \subset S^{2}\left(V^{*}\right) \cong \operatorname{Sym}(10)$ is represented as the symmetric matix of the following form;

$$
\left(\begin{array}{cccccccccc}
0 & 0 & 0 & 0 & 0 & 0 & 0 & a_{5} & a_{4} & a_{3} \\
0 & 0 & 0 & 0 & -a_{5} & 0 & -a_{4} & 0 & 0 & a_{2} \\
0 & 0 & 0 & a_{5} & 0 & a_{4} & 0 & 0 & 0 & a_{1} \\
0 & 0 & a_{5} & 0 & 0 & 0 & -a_{3} & 0 & -a_{2} & 0 \\
0 & -a_{5} & 0 & 0 & 0 & a_{3} & 0 & 0 & -a_{1} & 0 \\
0 & 0 & a_{4} & 0 & a_{3} & 0 & 0 & a_{2} & 0 & 0 \\
0 & -a_{4} & 0 & -a_{3} & 0 & 0 & 0 & a_{1} & 0 & 0 \\
a_{5} & 0 & 0 & 0 & 0 & a_{2} & a_{1} & 0 & 0 & 0 \\
a_{4} & 0 & 0 & -a_{2} & -a_{1} & 0 & 0 & 0 & 0 & 0 \\
a_{3} & a_{2} & a_{1} & 0 & 0 & 0 & 0 & 0 & 0 & 0
\end{array}\right)
$$

Hence the standard differential system $\left(M(\mathfrak{m}), D_{\mathfrak{m}}\right)$ of type $\mathfrak{m}$ in this case is given by

$$
D_{\mathfrak{m}}=\left\{\varpi=\varpi_{1}=\varpi_{2}=\cdots=\varpi_{10}=0\right\},
$$


where

$$
\begin{array}{rlrl}
\varpi & =d y-p_{1} d x_{1}-\cdots-p_{10} d x_{10}, & & \\
\varpi_{1}=d p_{1}+q_{5} d x_{8}+q_{4} d x_{9}+q_{3} d x_{10}, & & \varpi_{2}=d p_{2}-q_{5} d x_{5}-q_{4} d x_{7}+q_{2} d x_{10}, \\
\varpi_{3}=d p_{3}+q_{5} d x_{4}+q_{4} d x_{6}+q_{1} d x_{10}, & \varpi_{4}=d p_{4}+q_{5} d x_{3}-q_{3} d x_{7}-q_{2} d x_{9}, \\
\varpi_{5}=d p_{5}-q_{5} d x_{2}+q_{3} d x_{6}-q_{1} d x_{9}, & \varpi_{6}=d p_{6}+q_{4} d x_{3}+q_{3} d x_{5}+q_{2} d x_{8}, \\
\varpi_{7}=d p_{7}-q_{4} d x_{2}-q_{3} d x_{4}+q_{1} d x_{8}, & \varpi_{8}=d p_{8}+q_{5} d x_{1}+q_{2} d x_{6}+q_{1} d x_{7}, \\
\varpi_{9}=d p_{9}+q_{4} d x_{1}-q_{2} d x_{4}-q_{1} d x_{5} & \varpi_{10}=d p_{10}+q_{3} d x_{1}+q_{2} d x_{2}+q_{1} d x_{3} .
\end{array}
$$

Here $\left(x_{1}, \ldots, x_{10}, y, p_{1}, \ldots, p_{10}, q_{1}, \ldots, q_{5}\right)$ is a coordinate system of $M(\mathfrak{m}) \cong \mathbb{K}^{26}$. Thus the model equation of our typical symbol $\mathfrak{m}=\check{\mathfrak{g}}_{-3} \oplus \check{\mathfrak{g}}_{-2} \oplus \check{\mathfrak{g}}_{-1} \subset \mathfrak{C}^{2}\left(\mathfrak{l}_{-1}, \mathbb{K}\right)$ is given by

$$
\begin{array}{rlrl}
\frac{\partial^{2} y}{\partial x_{3} \partial x_{10}} & =-\frac{\partial^{2} y}{\partial x_{5} \partial x_{9}}=\frac{\partial^{2} y}{\partial x_{7} \partial x_{8}}, & & \frac{\partial^{2} y}{\partial x_{2} \partial x_{10}}=-\frac{\partial^{2} y}{\partial x_{4} \partial x_{9}}=\frac{\partial^{2} y}{\partial x_{6} \partial x_{8}} \\
\frac{\partial^{2} y}{\partial x_{1} \partial x_{10}}=-\frac{\partial^{2} y}{\partial x_{4} \partial x_{7}}=\frac{\partial^{2} y}{\partial x_{5} \partial x_{6}}, & \frac{\partial^{2} y}{\partial x_{1} \partial x_{9}}=-\frac{\partial^{2} y}{\partial x_{2} \partial x_{7}}=\frac{\partial^{2} y}{\partial x_{3} \partial x_{6}} \\
\frac{\partial^{2} y}{\partial x_{1} \partial x_{8}}=-\frac{\partial^{2} y}{\partial x_{2} \partial x_{5}}=\frac{\partial^{2} y}{\partial x_{3} \partial x_{4}}, & \frac{\partial^{2} y}{\partial x_{i} \partial x_{j}}=0 \quad \text { otherwise }
\end{array}
$$

where $y$ is dependent variable and $x_{1}, \ldots, x_{10}$ are independent variables. By a direct calculation, we see that the prolongation of the second order system (4.11) is given by

$$
\frac{\partial^{3} y}{\partial x_{i} \partial x_{j} \partial x_{k}}=0 \quad \text { for } \quad 1 \leqq i, j, k \leqq 10 .
$$

Case (4) $\left[\left(E_{6},\left\{\alpha_{6}\right\}\right), \varpi_{6},\left(E_{7},\left\{\gamma_{6}, \gamma_{7}\right\}\right)\right]$.

For the gradation of type $\left(E_{7},\left\{\gamma_{6}, \gamma_{7}\right\}\right)$, we have

$$
\begin{aligned}
& \Phi_{3}^{+}=\left\{\quad \alpha_{1}=012221, \quad \alpha_{2}=112221, \quad \alpha_{3}=122221,\right. \\
& \alpha_{4}=12 \underset{1}{3} 221, \quad \alpha_{5}=12 \underset{1}{3} 321, \quad \alpha_{6}=12 \underset{2}{3} 221, \quad \alpha_{7}=12 \underset{2}{3} 321, \\
& \left.\alpha_{8}=12 \underset{2}{4} 321, \quad \alpha_{9}=13 \underset{2}{4} 321, \quad \alpha_{10}=23 \underset{2}{4} 321\right\} \text {, } \\
& \Phi_{2}^{+}=\left\{\quad \eta_{1}=\underset{0}{0} \underset{0}{0} 11, \quad \eta_{2}=0000111, \quad \eta_{3}=00 \underset{0}{1} 111, \quad \eta_{4}=00 \frac{1111}{0},\right. \\
& \eta_{5}=01{ }_{0}^{1} 111, \quad \eta_{6}=01{ }_{1}^{1111}, \quad \eta_{7}={ }_{0}^{1}{ }_{0}^{1} 111, \quad \eta_{8}=11{ }_{1}^{1} 111, \\
& \eta_{9}=01{ }_{1}^{2} 111, \quad \eta_{10}=11{\underset{1}{1}}_{1}^{2} 11, \quad \eta_{11}=01 \underset{1}{2} 211, \quad \eta_{12}=12 \underset{1}{2} 111, \\
& \left.\eta_{13}=11 \underset{1}{2} 211, \quad \eta_{14}=12 \underset{1}{2} 211, \quad \eta_{15}=12 \underset{1}{3} 211, \quad \eta_{16}=12 \underset{2}{2} 211\right\},
\end{aligned}
$$$$
\Phi_{1}^{+}=\Psi^{6} \cup \Psi^{7}
$$$$
\Psi^{6}=\left\{\quad \xi_{1}=0000010, \quad \xi_{2}=0000110, \quad \xi_{3}=00 \underset{0}{1} 110, \quad \xi_{4}=001110,\right.
$$$$
\xi_{5}=01 \underset{0}{1} 110, \quad \xi_{6}=01 \underset{1}{1110}, \quad \xi_{7}=11 \underset{0}{1110}, \quad \xi_{8}=11 \underset{1}{1110},
$$$$
\xi_{9}=012110, \quad \xi_{10}=112110, \quad \xi_{11}=01 \underset{1}{2} 210, \quad \xi_{12}=122110,
$$$$
\left.\xi_{13}=11 \underset{1}{2} 210, \quad \xi_{14}=12 \underset{1}{2} 210, \quad \xi_{15}=12 \underset{1}{32} 10, \quad \xi_{16}=12 \underset{2}{32} 10 \quad\right\},
$$

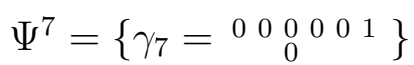

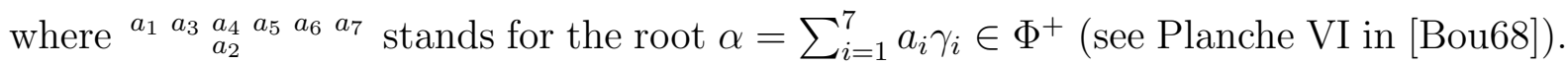

Thus we have $\mu=3$,

$$
\mathfrak{m}=\check{\mathfrak{g}}_{-3} \oplus \check{\mathfrak{g}}_{-2} \oplus \check{\mathfrak{g}}_{-1} \quad \text { and } \quad \check{\mathfrak{g}}_{-1}=S_{-1} \oplus \mathfrak{l}_{-1},
$$


where $\check{\mathfrak{g}}_{-3}=S_{-3}, \check{\mathfrak{g}}_{-2}=S_{-2}, S_{-1}$ and $\mathfrak{l}_{-1}$ are spanned by the root spaces $\mathfrak{g}_{-\beta}$ for $\beta \in$ $\Phi_{3}^{+}, \Phi_{2}^{+}, \Psi^{7}$ and $\Psi^{6}$ respectively. Hence $\operatorname{dim} S_{-3}=10, \operatorname{dim} S_{-2}=\operatorname{dim} \mathfrak{l}_{-1}=16$ and $\operatorname{dim} S_{-1}=1$.

For $\Phi_{3}^{+}, \Phi_{2}^{+}, \Psi^{7}$ and $\Psi^{6}$, we observe that $\alpha+\beta \notin \Phi$ for $\alpha, \beta \in \Phi_{3}^{+} \cup \Phi_{2}^{+} \cup \Psi^{7}, \xi-\gamma \notin \Phi$ for $\xi \in \Psi^{6}, \gamma \in \Psi^{7}$ and that, if $\eta+\xi \in \Phi_{3}^{+}$for $\eta \in \Phi_{2}^{+}, \xi \in \Psi^{6}$, then $\eta-\xi \notin \Phi$. This implies that $\left[y_{\alpha}, y_{\beta}\right]=0$ for $\alpha, \beta \in \Phi_{3}^{+} \cup \Phi_{2}^{+} \cup \Psi^{7},\left[y_{\gamma}, y_{\xi}\right]= \pm y_{\gamma+\xi}$ for $\xi \in \Psi^{6}, \gamma \in \Psi^{7}$, if $\gamma+\xi \in \Phi$ and $\left[y_{\eta}, y_{\xi}\right]= \pm y_{\eta+\xi}$ for $\eta \in \Phi_{2}^{+}, \xi \in \Psi^{6}$, if $\eta+\xi \in \Phi$, by the property of the Chevalley basis. Hence, from Planche VI in [Bou68], we readily obtain the non-trivial bracket relation among $\check{\mathfrak{g}}_{-1}$ and $\left[\check{\mathfrak{g}}_{-2}, \mathfrak{l}_{-1}\right]$ as in (4.13) and (4.14) below up to signs.

We fix the signs of $y_{\beta}$ for $\beta \in \Phi_{3}^{+}, \Phi_{2}^{+}, \Psi^{7}$ and $\Psi^{6}$ as follows: First we choose the orientation of $y_{\gamma_{i}}$ for simple roots by fixing the root vectors $y_{i}=y_{\gamma_{i}} \in \mathfrak{g}_{-\gamma_{i}}$. For $\xi \in \Psi^{6}$, we fix the orientation by the following order;

$$
\begin{aligned}
& y_{\xi_{1}}=y_{6}, \quad y_{\xi_{2}}=\left[y_{\xi_{1}}, y_{5}\right], \quad y_{\xi_{3}}=\left[y_{\xi_{2}}, y_{4}\right], \quad y_{\xi_{4}}=\left[y_{\xi_{3}}, y_{2}\right], \\
& y_{\xi_{5}}=\left[y_{\xi_{3}}, y_{3}\right], \quad y_{\xi_{6}}=\left[y_{\xi_{5}}, y_{2}\right], \quad y_{\xi_{7}}=\left[y_{\xi_{5}}, y_{1}\right], \quad y_{\xi_{8}}=\left[y_{\xi_{7}}, y_{2}\right], \\
& y_{\xi_{9}}=\left[y_{\xi_{6}}, y_{4}\right], \quad y_{\xi_{10}}=\left[y_{\xi_{9}}, y_{1}\right], \quad y_{\xi_{11}}=\left[y_{\xi_{9}}, y_{5}\right], \quad y_{\xi_{12}}=\left[y_{\xi_{10}}, y_{3}\right], \\
& y_{\xi_{13}}=\left[y_{\xi_{11}}, y_{1}\right], \quad y_{\xi_{14}}=\left[y_{\xi_{13}}, y_{3}\right], \quad y_{\xi_{15}}=\left[y_{\xi_{14}}, y_{4}\right], \quad y_{\xi_{16}}=\left[y_{\xi_{15}}, y_{2}\right] \text {. }
\end{aligned}
$$

For $\eta \in \Phi_{2}^{+}$, we fix the orientation by the following ;

$$
y_{\eta_{p}}=\left[y_{7}, y_{\xi_{p}}\right] \quad \text { for } \quad p=1,2, \ldots, 16 .
$$

Finally, for $\alpha \in \Phi_{3}^{+}$, we fix the orientation by the following;

$$
\begin{aligned}
& y_{\alpha_{1}}=\left[y_{\eta_{11}}, y_{\xi_{1}}\right], \quad y_{\alpha_{2}}=\left[y_{\eta_{13}}, y_{\xi_{1}}\right], \quad y_{\alpha_{3}}=\left[y_{\eta_{14}}, y_{\xi_{1}}\right], \quad y_{\alpha_{4}}=\left[y_{\eta_{15}}, y_{\xi_{1}}\right], \\
& y_{\alpha_{5}}=\left[y_{\eta_{15}}, y_{\xi_{2}}\right], \quad y_{\alpha_{6}}=\left[y_{\eta_{16}}, y_{\xi_{1}}\right], \quad y_{\alpha_{7}}=\left[y_{\eta_{16}}, y_{\xi_{2}}\right], \quad y_{\alpha_{8}}=\left[y_{\eta_{16}}, y_{\xi_{3}}\right] \text {, } \\
& y_{\alpha_{9}}=\left[y_{\eta_{16}}, y_{\xi_{5}}\right], \quad y_{\alpha_{10}}=\left[y_{\eta_{16}}, y_{\xi_{7}}\right] \text {, }
\end{aligned}
$$

Then, for example, we calculate

$$
\left[y_{\eta_{p}}, y_{\xi_{q}}\right]=\left[\left[y_{7}, y_{\xi_{p}}\right], y_{\xi_{q}}\right]=\left[\left[y_{7}, y_{\xi_{q}}\right], y_{\xi_{p}}\right]=\left[y_{\eta_{q}}, y_{\xi_{p}}\right] \quad \text { for } \quad 1 \leqq p, q \leqq 16,
$$

and

$$
\begin{array}{r}
{\left[y_{\eta_{11}}, y_{\xi_{1}}\right]=\left[\left[y_{7}, y_{\xi_{11}}\right], y_{\xi_{1}}\right]=\left[\left[y_{7},\left[y_{\xi_{9}}, y_{5}\right]\right], y_{\xi_{1}}\right]=\left[\left[\left[y_{7}, y_{\xi_{9}}\right], y_{5}\right], y_{\xi_{1}}\right]} \\
=\left[\left[y_{\eta_{9}}, y_{5}\right], y_{\xi_{1}}\right]=\left[y_{\eta_{9}},\left[y_{5}, y_{\xi_{1}}\right]\right]=-\left[y_{\eta_{9}}, y_{\xi_{2}}\right] .
\end{array}
$$

In the same way, by the repeated application of Jacobi identities, we obtain

$$
\begin{gathered}
y_{\alpha_{1}}=\left[y_{\eta_{11}}, y_{\xi_{1}}\right]=-\left[y_{\eta_{9}}, y_{\xi_{2}}\right]=\left[y_{\eta_{6}}, y_{\xi_{3}}\right]=-\left[y_{\eta_{5}}, y_{\xi_{4}}\right], \\
y_{\alpha_{2}}=\left[y_{\eta_{13}}, y_{\xi_{1}}\right]=-\left[y_{\eta_{10}}, y_{\xi_{2}}\right]=\left[y_{\eta_{8}}, y_{\xi_{3}}\right]=-\left[y_{\eta_{7}}, y_{\xi_{4}}\right], \\
y_{\alpha_{3}}=\left[y_{\eta_{14}}, y_{\xi_{1}}\right]=-\left[y_{\eta_{12}}, y_{\xi_{2}}\right]=\left[y_{\eta_{8}}, y_{\xi_{5}}\right]=-\left[y_{\eta_{7}}, y_{\xi_{6}}\right], \\
y_{\alpha_{4}}=\left[y_{\eta_{15}}, y_{\xi_{1}}\right]=-\left[y_{\eta_{12}}, y_{\xi_{3}}\right]=\left[y_{\eta_{10}}, y_{\xi_{5}}\right]=-\left[y_{\eta_{9}}, y_{\xi_{7}}\right], \\
y_{\alpha_{5}}=\left[y_{\eta_{15}}, y_{\xi_{2}}\right]=-\left[y_{\eta_{14}}, y_{\xi_{3}}\right]=\left[y_{\eta_{13}}, y_{\xi_{5}}\right]=-\left[y_{\eta_{11}}, y_{\xi_{7}}\right], \\
y_{\alpha_{6}}=\left[y_{\eta_{16}}, y_{\xi_{1}}\right]=-\left[y_{\eta_{12}}, y_{\xi_{4}}\right]=\left[y_{\eta_{10}}, y_{\xi_{6}}\right]=-\left[y_{\eta_{9}}, y_{\xi_{8}}\right], \\
y_{\alpha_{7}}=\left[y_{\eta_{16}}, y_{\xi_{2}}\right]=-\left[y_{\eta_{14}}, y_{\xi_{4}}\right]=\left[y_{\eta_{13}}, y_{\xi_{6}}\right]=-\left[y_{\eta_{11}}, y_{\xi_{8}}\right], \\
y_{\alpha_{8}}=\left[y_{\eta_{16}}, y_{\xi_{3}}\right]=-\left[y_{\eta_{15}}, y_{\xi_{4}}\right]=\left[y_{\eta_{13}}, y_{\xi_{9}}\right]=-\left[y_{\eta_{11}}, y_{\xi_{10}}\right], \\
y_{\alpha_{9}}=\left[y_{\eta_{16}}, y_{\xi_{5}}\right]=-\left[y_{\eta_{15}}, y_{\xi_{6}}\right]=\left[y_{\eta_{14}}, y_{\xi_{9}}\right]=-\left[y_{\eta_{12}}, y_{\xi_{11}}\right], \\
y_{\alpha_{10}}=\left[y_{\eta_{16}}, y_{\xi_{7}}\right]=-\left[y_{\eta_{15}}, y_{\xi_{8}}\right]=\left[y_{\eta_{14}}, y_{\xi_{10}}\right]=-\left[y_{\eta_{13}}, y_{\xi_{12}}\right] .
\end{gathered}
$$


Then, by fixing the basis $\left\{y_{\alpha_{i}}\right\}_{i=1}^{10}$ of $S_{-3}$ and $\left\{y_{\xi_{j}}\right\}_{j=1}^{16}$ of $\mathfrak{l}_{-1}$, an element $A=\sum_{j=1}^{16} a_{j} \operatorname{ad}\left(y_{\eta_{j}}\right)$ $\in S_{-2} \subset S_{-3} \otimes\left(\mathfrak{l}_{-1}\right)^{*} \cong M(10,16)$ is represented as the matrix of the following form;

$$
\left(\begin{array}{cccccccccccccccc}
a_{11} & a_{9}{ }^{*} & a_{6} & a_{5}{ }^{*} & a_{4}{ }^{*} & a_{3} & 0 & 0 & a_{2}{ }^{*} & 0 & a_{1} & 0 & 0 & 0 & 0 & 0 \\
a_{13} & a_{10}{ }^{*} & a_{8} & a_{7}{ }^{*} & 0 & 0 & a_{4}{ }^{*} & a_{3} & 0 & a_{2}{ }^{*} & 0 & 0 & a_{1} & 0 & 0 & 0 \\
a_{14} & a_{12}{ }^{*} & 0 & 0 & a_{8} & a_{7}{ }^{*} & a_{6}{ }^{*} & a_{5} & 0 & 0 & 0 & a_{2}{ }^{*} & 0 & a_{1} & 0 & 0 \\
a_{15} & 0 & a_{12}{ }^{*} & 0 & a_{10} & 0 & a_{9}{ }^{*} & 0 & a_{7}{ }^{*} & a_{5} & 0 & a_{3}{ }^{*} & 0 & 0 & a_{1} & 0 \\
0 & a_{15} & a_{14}{ }^{*} & 0 & a_{13} & 0 & a_{11}{ }^{*} & 0 & 0 & 0 & a_{7}{ }^{*} & 0 & a_{5} & a_{3}{ }^{*} & a_{2} & 0 \\
a_{16} & 0 & 0 & a_{12}{ }^{*} & 0 & a_{10} & 0 & a_{9}{ }^{*} & a_{8}{ }^{*} & a_{6} & 0 & a_{4}{ }^{*} & 0 & 0 & 0 & a_{1} \\
0 & a_{16} & 0 & a_{14}{ }^{*} & 0 & a_{13} & 0 & a_{11}{ }^{*} & 0 & 0 & a_{8}{ }^{*} & 0 & a_{6} & a_{4}{ }^{*} & 0 & a_{2} \\
0 & 0 & a_{16} & a_{15}{ }^{*} & 0 & 0 & 0 & 0 & a_{13} & a_{11}{ }^{*} & a_{10}{ }^{*} & 0 & a_{9} & 0 & a_{4}{ }^{*} & a_{3} \\
0 & 0 & 0 & 0 & a_{16} & a_{15}{ }^{*} & 0 & 0 & a_{14} & 0 & a_{12}{ }^{*} & a_{11}{ }^{*} & 0 & a_{9} & a_{6}{ }^{*} & a_{5} \\
0 & 0 & 0 & 0 & 0 & 0 & a_{16} & a_{15}{ }^{*} & 0 & a_{14} & 0 & a_{13}{ }^{*} & a_{12}{ }^{*} & a_{10} & a_{8}{ }^{*} & a_{7}
\end{array}\right)
$$

where $a_{i}{ }^{*}=-a_{i}$. Moreover, for $y_{7} \in S_{-1}$, we have

$$
\begin{aligned}
y_{\alpha_{1}}=\left[\left[y_{7}, y_{\xi_{1}}\right], y_{\xi_{11}}\right]=-\left[\left[y_{7} \cdot y_{\xi_{2}}\right], y_{\xi_{9}}\right]=\left[\left[y_{7}, y_{\xi_{3}}\right], y_{\xi_{6}}\right]=-\left[\left[y_{7} \cdot y_{\xi_{4}}\right], y_{\xi_{5}}\right], \\
y_{\alpha_{2}}=\left[\left[y_{7}, y_{\xi_{1}}\right], y_{\xi_{13}}\right]=-\left[\left[y_{7}, y_{\xi_{2}}\right], y_{\xi_{10}}\right]=\left[\left[y_{7}, y_{\xi_{3}}\right], y_{\xi_{8}}\right]=-\left[\left[y_{7}, y_{\xi_{4}}\right], y_{\xi_{7}}\right], \\
y_{\alpha_{3}}=\left[\left[y_{7}, y_{\xi_{1}}\right], y_{\xi_{14}}\right]=-\left[\left[y_{7}, y_{\xi_{2}}\right], y_{\xi_{12}}\right]=\left[\left[y_{7}, y_{\xi_{5}}\right], y_{\xi_{8}}\right]=-\left[\left[y_{7}, y_{\xi_{6}}\right], y_{\xi_{7}}\right], \\
y_{\alpha_{4}}=\left[\left[y_{7}, y_{\xi_{1}}\right], y_{\xi_{15}}\right]=-\left[\left[y_{7}, y_{\xi_{3}}\right], y_{\xi_{12}}\right]=\left[\left[y_{7}, y_{\xi_{5}}\right], y_{\xi_{10}}\right]=-\left[\left[y_{7}, y_{\xi_{7}}\right], y_{\xi_{9}}\right], \\
y_{\alpha_{5}}=\left[\left[y_{7}, y_{\xi_{2}}\right], y_{\xi_{15}}\right]=-\left[\left[y_{7}, y_{\xi_{3}}\right], y_{\xi_{14}}\right]=\left[\left[y_{7}, y_{\xi_{5}}\right], y_{\xi_{13}}\right]=-\left[\left[y_{7}, y_{\xi_{7}}\right], y_{\xi_{11}}\right], \\
y_{\alpha_{6}}=\left[\left[y_{7}, y_{\xi_{1}}\right], y_{\xi_{16}}\right]=-\left[\left[y_{7} \cdot y_{\xi_{4}}\right], y_{\xi_{12}}\right]=\left[\left[y_{7}, y_{\xi_{6}}\right], y_{\xi_{10}}\right]=-\left[\left[y_{7} . y_{\xi_{8}}\right], y_{\xi_{9}}\right], \\
y_{\alpha_{7}}=\left[\left[y_{7}, y_{\xi_{2}}\right], y_{\xi_{16}}\right]=-\left[\left[y_{7}, y_{\xi_{4}}\right], y_{\xi_{14}}\right]=\left[\left[y_{7}, y_{\xi_{6}}\right], y_{\xi_{13}}\right]=-\left[\left[y_{7}, y_{\xi_{8}}\right], y_{\xi_{11}}\right], \\
y_{\alpha_{8}}=\left[\left[y_{7}, y_{\xi_{3}}\right], y_{\xi_{16}}\right]=-\left[\left[y_{7}, y_{\xi_{4}}\right], y_{\xi_{15}}\right]=\left[\left[y_{7}, y_{\xi_{9}}\right], y_{\xi_{13}}\right]=-\left[\left[y_{7}, y_{\xi_{10}}\right], y_{\xi_{11}}\right], \\
y_{\alpha_{9}}=\left[\left[y_{7}, y_{\xi_{5}}\right], y_{\xi_{16}}\right]=-\left[\left[y_{7}, y_{\xi_{6}}\right], y_{\xi_{15}}\right]=\left[\left[y_{7}, y_{\xi_{9}}\right], y_{\xi_{14}}\right]=-\left[\left[y_{7}, y_{\xi_{11}}\right], y_{\xi_{12}}\right], \\
y_{\alpha_{10}}=\left[\left[y_{7}, y_{\xi_{7}}\right], y_{\xi_{16}}\right]=-\left[\left[y_{7}, y_{\xi_{8}}\right], y_{\xi_{15}}\right]=\left[\left[y_{7}, y_{\xi_{10}}\right], y_{\xi_{14}}\right]=-\left[\left[y_{7}, y_{\xi_{12}}\right], y_{\xi_{13}}\right] .
\end{aligned}
$$

Thus $S_{-1}$ is embedded as the 1-dimensional subspace of $S_{-3} \otimes S^{2}\left(\left(\mathfrak{l}_{-1}\right)^{*}\right)$ spanned by the following quadratic form $f$

$$
\begin{aligned}
f(X, X) & =\left(x_{1} x_{11}-x_{2} x_{9}+x_{3} x_{6}-x_{4} x_{5}\right) y_{\alpha_{1}}+\left(x_{1} x_{13}-x_{2} x_{10}+x_{3} x_{8}-x_{4} x_{7}\right) y_{\alpha_{2}} \\
& +\left(x_{1} x_{14}-x_{2} x_{12}+x_{5} x_{8}-x_{6} x_{7}\right) y_{\alpha_{3}}+\left(x_{1} x_{15}-x_{3} x_{12}+x_{5} x_{10}-x_{7} x_{9}\right) y_{\alpha_{4}} \\
+ & \left(x_{2} x_{15}-x_{3} x_{14}+x_{5} x_{13}-x_{7} x_{11}\right) y_{\alpha_{5}}+\left(x_{1} x_{16}-x_{4} x_{12}+x_{6} x_{10}-x_{8} x_{9}\right) y_{\alpha_{6}} \\
+ & \left(x_{2} x_{16}-x_{4} x_{14}+x_{6} x_{13}-x_{8} x_{11}\right) y_{\alpha_{7}}+\left(x_{3} x_{16}-x_{4} x_{15}+x_{9} x_{13}-x_{10} x_{11}\right) y_{\alpha_{8}} \\
& +\left(x_{5} x_{16}-x_{6} x_{15}+x_{9} x_{14}-x_{11} x_{12}\right) y_{\alpha_{9}}+\left(x_{7} x_{16}-x_{8} x_{15}+x_{10} x_{14}-x_{12} x_{13}\right) y_{\alpha_{10}}
\end{aligned}
$$

for $X=\sum_{j=1}^{16} x_{j} y_{\xi_{j}} \in \mathfrak{l}_{-1}$.

By the above matrix representation, we can embed $S_{-2}$ as a subspace of $S_{-3} \otimes\left(\mathfrak{l}_{-1}\right)^{*} \cong$ $M(10,16)$ and obtain the following first order system as the model equation whose symbol coincides with this subspace:

$$
\begin{array}{rlrlrl}
\frac{\partial y_{1}}{\partial x_{11}} & =\frac{\partial y_{2}}{\partial x_{13}}=\frac{\partial y_{3}}{\partial x_{14}}=\frac{\partial y_{4}}{\partial x_{15}}=\frac{\partial y_{6}}{\partial x_{16}}, & -\frac{\partial y_{1}}{\partial x_{9}}=-\frac{\partial y_{2}}{\partial x_{10}}=-\frac{\partial y_{3}}{\partial x_{12}}=\frac{\partial y_{5}}{\partial x_{15}}=\frac{\partial y_{7}}{\partial x_{16}} \\
\frac{\partial y_{1}}{\partial x_{6}}=\frac{\partial y_{2}}{\partial x_{8}}=-\frac{\partial y_{4}}{\partial x_{12}}=-\frac{\partial y_{5}}{\partial x_{14}}=\frac{\partial y_{8}}{\partial x_{16}}, & \frac{\partial y_{1}}{\partial x_{5}}=\frac{\partial y_{2}}{\partial x_{7}}=\frac{\partial y_{6}}{\partial x_{12}}=\frac{\partial y_{7}}{\partial x_{14}}=\frac{\partial y_{8}}{\partial x_{15}}, \\
-\frac{\partial y_{1}}{\partial x_{4}} & =\frac{\partial y_{3}}{\partial x_{8}}=\frac{\partial y_{4}}{\partial x_{10}}=\frac{\partial y_{5}}{\partial x_{13}}=\frac{\partial y_{9}}{\partial x_{16}}, & \frac{\partial y_{1}}{\partial x_{3}} & =-\frac{\partial y_{3}}{\partial x_{7}}=\frac{\partial y_{6}}{\partial x_{10}}=\frac{\partial y_{7}}{\partial x_{13}}=-\frac{\partial y_{9}}{\partial x_{15}},
\end{array}
$$




$$
\begin{array}{rlrlrl}
-\frac{\partial y_{2}}{\partial x_{4}} & =-\frac{\partial y_{3}}{\partial x_{6}}=-\frac{\partial y_{4}}{\partial x_{9}}=-\frac{\partial y_{5}}{\partial x_{11}}=\frac{\partial y_{10}}{\partial x_{16}}, & & \frac{\partial y_{2}}{\partial x_{3}}=\frac{\partial y_{3}}{\partial x_{5}}=-\frac{\partial y_{6}}{\partial x_{9}}=-\frac{\partial y_{7}}{\partial x_{11}}=-\frac{\partial y_{10}}{\partial x_{15}}, \\
-\frac{\partial y_{1}}{\partial x_{2}} & =-\frac{\partial y_{4}}{\partial x_{7}}=-\frac{\partial y_{6}}{\partial x_{8}}=\frac{\partial y_{8}}{\partial x_{13}}=\frac{\partial y_{9}}{\partial x_{14}}, & -\frac{\partial y_{2}}{\partial x_{2}}=\frac{\partial y_{4}}{\partial x_{5}}=\frac{\partial y_{6}}{\partial x_{6}}=-\frac{\partial y_{8}}{\partial x_{11}}=\frac{\partial y_{10}}{\partial x_{14}} \\
\frac{\partial y_{1}}{\partial x_{1}}=-\frac{\partial y_{5}}{\partial x_{7}}=-\frac{\partial y_{7}}{\partial x_{8}}=-\frac{\partial y_{8}}{\partial x_{10}}=-\frac{\partial y_{9}}{\partial x_{12}}, & \frac{\partial y_{3}}{\partial x_{2}}=\frac{\partial y_{4}}{\partial x_{3}}=\frac{\partial y_{6}}{\partial x_{4}}=\frac{\partial y_{9}}{\partial x_{11}}=\frac{\partial y_{10}}{\partial x_{13}}, \\
\frac{\partial y_{2}}{\partial x_{1}}=\frac{\partial y_{5}}{\partial x_{5}}=\frac{\partial y_{7}}{\partial x_{6}}=\frac{\partial y_{8}}{\partial x_{9}}=-\frac{\partial y_{10}}{\partial x_{12}}, & \frac{\partial y_{3}}{\partial x_{1}}=-\frac{\partial y_{5}}{\partial x_{3}}=-\frac{\partial y_{7}}{\partial x_{4}}=\frac{\partial y_{9}}{\partial x_{9}}=\frac{\partial y_{10}}{\partial x_{10}}, \\
\frac{\partial y_{4}}{\partial x_{1}} & =\frac{\partial y_{5}}{\partial x_{2}}=-\frac{\partial y_{8}}{\partial x_{4}}=-\frac{\partial y_{9}}{\partial x_{6}}=-\frac{\partial y_{10}}{\partial x_{8}}, & & \frac{\partial y_{6}}{\partial x_{1}}=\frac{\partial y_{7}}{\partial x_{2}}=\frac{\partial y_{8}}{\partial x_{3}}=\frac{\partial y_{9}}{\partial x_{5}}=\frac{\partial y_{10}}{\partial x_{7}}, \\
\frac{\partial y_{i}}{\partial x_{j}} & =0 & & \text { otherwise }
\end{array}
$$

where $y_{1}, \ldots, y_{10}$ are dependent variables and $x_{1}, \ldots, x_{16}$ are independent variables. Moreover, by a direct calculation, we see that the prolongation of the first order system (4.15) is given by

$$
\begin{aligned}
& \frac{\partial^{2} y_{1}}{\partial x_{1} \partial x_{11}}=\frac{\partial^{2} y_{2}}{\partial x_{1} \partial x_{13}}=\frac{\partial^{2} y_{3}}{\partial x_{1} \partial x_{14}}=\frac{\partial^{2} y_{4}}{\partial x_{1} \partial x_{15}}=\frac{\partial^{2} y_{6}}{\partial x_{1} \partial x_{16}}=\frac{\partial^{2} y_{7}}{\partial x_{16} \partial x_{2}} \\
&= \frac{\partial^{2} y_{8}}{\partial x_{16} \partial x_{3}}=\frac{\partial^{2} y_{9}}{\partial x_{16} \partial x_{5}}=\frac{\partial^{2} y_{10}}{\partial x_{16} \partial x_{7}}=-\frac{\partial^{2} y_{5}}{\partial x_{7} \partial x_{11}}=-\frac{\partial^{2} y_{4}}{\partial x_{7} \partial x_{9}}=-\frac{\partial^{2} y_{3}}{\partial x_{7} \partial x_{6}} \\
&=-\frac{\partial^{2} y_{2}}{\partial x_{7} \partial x_{4}}=-\frac{\partial^{2} y_{6}}{\partial x_{4} \partial x_{12}}=-\frac{\partial^{2} y_{7}}{\partial x_{4} \partial x_{14}}=-\frac{\partial^{2} y_{8}}{\partial x_{4} \partial x_{15}}=-\frac{\partial^{2} y_{1}}{\partial x_{4} \partial x_{5}}=\frac{\partial^{2} y_{3}}{\partial x_{5} \partial x_{8}} \\
&= \frac{\partial^{2} y_{4}}{\partial x_{5} \partial x_{10}}=\frac{\partial^{2} y_{5}}{\partial x_{5} \partial x_{13}}=\frac{\partial^{2} y_{7}}{\partial x_{13} \partial x_{6}}=\frac{\partial^{2} y_{8}}{\partial x_{13} \partial x_{9}}=-\frac{\partial^{2} y_{10}}{\partial x_{13} \partial x_{12}}=-\frac{\partial^{2} y_{9}}{\partial x_{12} \partial x_{11}} \\
&=- \frac{\partial^{2} y_{4}}{\partial x_{12} \partial x_{3}}=-\frac{\partial^{2} y_{3}}{\partial x_{12} \partial x_{2}}=\frac{\partial^{2} y_{5}}{\partial x_{2} \partial x_{15}}=-\frac{\partial^{2} y_{2}}{\partial x_{2} \partial x_{10}}=-\frac{\partial^{2} y_{1}}{\partial x_{2} \partial x_{9}}=-\frac{\partial^{2} y_{6}}{\partial x_{9} \partial x_{8}} \\
&= \frac{\partial^{2} y_{9}}{\partial x_{9} \partial x_{14}}=-\frac{\partial^{2} y_{5}}{\partial x_{14} \partial x_{3}}=\frac{\partial^{2} y_{10}}{\partial x_{14} \partial x_{10}}=-\frac{\partial^{2} y_{8}}{\partial x_{10} \partial x_{11}}=\frac{\partial^{2} y_{6}}{\partial x_{10} \partial x_{6}}=-\frac{\partial^{2} y_{9}}{\partial x_{6} \partial x_{15}} \\
&= \frac{\partial^{2} y_{1}}{\partial x_{6} \partial x_{3}}=\frac{\partial^{2} y_{2}}{\partial x_{3} \partial x_{8}}=-\frac{\partial^{2} y_{7}}{\partial x_{8} \partial x_{11}}=-\frac{\partial^{2} y_{10}}{\partial x_{8} \partial x_{15}}, \\
& \frac{\partial^{2} y_{i}}{\partial x_{p} \partial x_{q}}=0 \\
& \text { otherwise. }
\end{aligned}
$$

From the above expression of $f$, we observe that the above second order system is the model equation of the 1-dimensional embedded subspace $S_{-1}$ in $S_{-3} \otimes S^{2}\left(\left(\mathfrak{l}_{-1}\right)^{*}\right)$.Furthermore, by a direct calculation, we see that the prolongation of this second order system (4.16) is given by

$$
\frac{\partial^{3} y_{i}}{\partial x_{p} \partial x_{q} \partial x_{r}}=0 \quad \text { for } \quad 1 \leqq i \leqq 10, \quad 1 \leqq p, q, r \leqq 16
$$

Case $(5)\left[\left(E_{6},\left\{\alpha_{1}\right\}\right), \varpi_{6},\left(E_{7},\left\{\gamma_{1}, \gamma_{7}\right\}\right)\right]$.

For the gradation of type $\left(E_{7},\left\{\gamma_{1}, \gamma_{7}\right\}\right)$, we have 


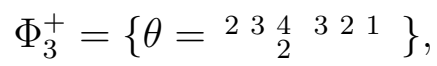

$$
\begin{aligned}
& \Phi_{2}^{+}=\left\{\quad \eta_{1}=13 \underset{2}{4} 321, \quad \eta_{2}=12 \underset{2}{4} 321, \quad \eta_{3}=12 \underset{2}{3} 321, \quad \eta_{4}=12 \underset{1}{3} 321,\right. \\
& \eta_{5}=12 \underset{2}{3} 221, \quad \eta_{6}=12 \underset{1}{3} 221, \quad \eta_{7}=12 \underset{2}{3} 211, \quad \eta_{8}=12 \underset{1}{3} 211, \\
& \eta_{9}=12 \underset{1}{2} 221, \quad \eta_{10}=12 \underset{1}{2} 211, \quad \eta_{11}=11 \underset{1}{2} 221, \quad \eta_{12}=1{ }_{1}^{2} 211,
\end{aligned}
$$

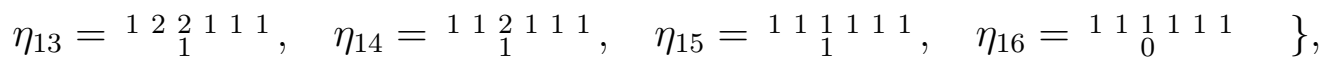

$$
\begin{aligned}
& \Phi_{1}^{+}=\Psi^{1} \cup \Psi^{7} \\
& \Psi^{1}=\left\{\quad \xi_{1}=1000000, \quad \xi_{2}=110000, \quad \xi_{3}=11 \underset{0}{10000}, \quad \xi_{4}=11 \underset{1}{10000},\right. \\
& \xi_{5}=11 \underset{0}{1} 100, \quad \xi_{6}=11{ }_{1}^{1100}, \quad \xi_{7}=11{ }_{0}^{1110}, \quad \xi_{8}=11{ }_{1}^{1110}, \\
& \xi_{9}=112100, \quad \xi_{10}=112110, \quad \xi_{11}=122100, \quad \xi_{12}=122110, \\
& \left.\xi_{13}=11 \underset{1}{2} 210, \quad \xi_{14}=12 \underset{1}{2} 210, \quad \xi_{15}=12 \underset{1}{32} 10, \quad \xi_{16}=12 \underset{2}{3} 210 \quad\right\} \text {, } \\
& \Psi^{7}=\left\{\quad \zeta_{1}=000001, \quad \zeta_{2}=000011, \quad \zeta_{3}=0000111,\right. \\
& \zeta_{4}=001111, \quad \zeta_{5}=011111, \quad \zeta_{6}=001111, \quad \zeta_{7}=011111,
\end{aligned}
$$

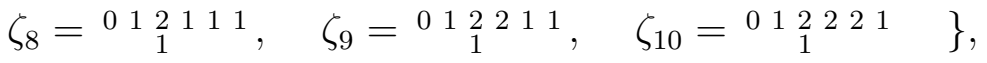

where ${ }^{a_{1}} a_{3} a_{3} a_{4} a_{5} a_{6} a_{7}$ stands for the root $\alpha=\sum_{i=1}^{7} a_{i} \gamma_{i} \in \Phi^{+}$(see Planche VI in [Bou68]).

Thus we have $\mu=3$,

$$
\mathfrak{m}=\check{\mathfrak{g}}_{-3} \oplus \check{\mathfrak{g}}_{-2} \oplus \check{\mathfrak{g}}_{-1} \quad \text { and } \quad \check{\mathfrak{g}}_{-1}=S_{-1} \oplus \mathfrak{l}_{-1},
$$

where $\check{\mathfrak{g}}_{-3}=S_{-3}, \check{\mathfrak{g}}_{-2}=S_{-2}, S_{-1}$ and $\mathfrak{l}_{-1}$ are spanned by the root spaces $\mathfrak{g}_{-\beta}$ for $\beta \in \Phi_{3}^{+}, \Phi_{2}^{+}, \Psi^{7}$ and $\Psi^{1}$ respectively. Hence $\operatorname{dim} S_{-3}=1, \operatorname{dim} S_{-2}=\operatorname{dim} \mathfrak{l}_{-1}=16$ and $\operatorname{dim} S_{-1}=10$.

For $\Phi_{3}^{+}, \Phi_{2}^{+}, \Psi^{7}$ and $\Psi^{1}$, we observe that $\Phi_{3}^{+}=\{\theta\}$, where $\theta$ is the highest root, $\alpha+\beta \notin \Phi$ for $\alpha, \beta \in \Phi_{3}^{+} \cup \Phi_{2}^{+} \cup \Psi^{7}, \zeta-\xi \notin \Phi$ for $\xi \in \Psi^{1}, \zeta \in \Psi^{7}$ and that $\eta_{i}+\xi_{i}=\theta, \eta_{i}-\xi_{i} \notin \Phi$, $\xi_{i}+\xi_{j} \notin \Phi$ and $\eta_{i}+\xi_{j} \notin \Phi$ if $i \neq j$ for $\eta_{i} \in \Phi_{2}^{+}$and $\xi_{i}, \xi_{j} \in \Psi^{2}(i, j=1, \ldots, 16)$. This implies that $\left[y_{\alpha}, y_{\beta}\right]=0$ for $\alpha, \beta \in \Phi_{3}^{+} \cup \Phi_{2}^{+} \cup \Psi^{7},\left[y_{\zeta}, y_{\xi}\right]= \pm y_{\zeta+\xi}$ for $\xi \in \Psi^{2}, \zeta \in \Psi^{7}$, if $\zeta+\xi \in \Phi$ and that $\left[y_{\xi_{i}}, y_{\xi_{j}}\right]=0,\left[y_{\eta_{i}}, y_{\xi_{j}}\right]= \pm \delta_{i j} y_{\theta}$ for $\eta_{i} \in \Phi_{2}^{+}, \xi_{i}, \xi_{j} \in \Psi^{2}(i, j=1, \ldots, 16)$, by the property of the Chevalley basis. Hence, from Planche VI in [Bou68], we readily obtain the non-trivial bracket relation among $\check{\mathfrak{g}}_{-1}$ and $\left[\check{\mathfrak{g}}_{-2}, \mathfrak{l}_{-1}\right]$ as in (4.18) and (4.19) below up to signs.

We fix the signs of $y_{\beta}$ for $\beta \in \Phi_{3}^{+}, \Phi_{2}^{+}, \Psi^{7}$ and $\Psi^{1}$ as follows: First we choose the orientation of $y_{\gamma_{i}}$ for simple roots by fixing the root vectors $y_{i}=y_{\gamma_{i}} \in \mathfrak{g}_{-\gamma_{i}}$. For $\zeta \in \Psi^{7}$, we fix the orientation by the following order;

$$
\begin{aligned}
& y_{\zeta_{1}}=y_{7}, \quad y_{\zeta_{2}}=\left[y_{6}, y_{\zeta_{1}}\right], \quad y_{\zeta_{3}}=\left[y_{5}, y_{\zeta_{2}}\right], \quad y_{\zeta_{4}}=\left[y_{4}, y_{\zeta_{3}}\right], \quad y_{\zeta_{5}}=\left[y_{3}, y_{\zeta_{4}}\right], \\
& y_{\zeta_{6}}=\left[y_{2}, y_{\zeta_{4}}\right], \quad y_{\zeta_{7}}=\left[y_{2}, y_{\zeta_{5}}\right], \quad y_{\zeta_{8}}=\left[y_{4}, y_{\zeta_{7}}\right], \quad y_{\zeta_{9}}=\left[y_{5}, y_{\zeta_{8}}\right], \quad y_{\zeta_{10}}=\left[y_{6}, y_{\zeta_{9}}\right] \text {. }
\end{aligned}
$$

For $\xi \in \Psi^{1}$, we fix the orientation by the following order;

$$
\begin{aligned}
& y_{\xi_{1}}=y_{1}, \quad y_{\xi_{2}}=\left[y_{\xi_{1}}, y_{3}\right], \quad y_{\xi_{3}}=\left[y_{\xi_{2}}, y_{4}\right], \quad y_{\xi_{4}}=\left[y_{\xi_{3}}, y_{2}\right], \\
& y_{\xi_{5}}=\left[y_{\xi_{3}}, y_{5}\right], \quad y_{\xi_{6}}=\left[y_{\xi_{5}}, y_{2}\right], \quad y_{\xi_{7}}=\left[y_{\xi_{5}}, y_{6}\right], \quad y_{\xi_{8}}=\left[y_{\xi_{7}}, y_{2}\right], \\
& y_{\xi_{9}}=\left[y_{\xi_{6}}, y_{4}\right], \quad y_{\xi_{10}}=\left[y_{\xi_{9}}, y_{6}\right], \quad y_{\xi_{11}}=\left[y_{\xi_{9}}, y_{3}\right], \quad y_{\xi_{12}}=\left[y_{\xi_{11}}, y_{6}\right], \\
& y_{\xi_{13}}=\left[y_{\xi_{10}}, y_{5}\right], \quad y_{\xi_{14}}=\left[y_{\xi_{13}}, y_{3}\right], \quad y_{\xi_{15}}=\left[y_{\xi_{14}}, y_{4}\right], \quad y_{\xi_{16}}=\left[y_{\xi_{15}}, y_{2}\right] \text {. }
\end{aligned}
$$

For $\eta \in \Phi_{2}^{+}$, we fix the orientation by the following order;

$$
y_{\eta_{1}}=-\left[y_{\zeta_{5}}, y_{\xi_{16}}\right], \quad y_{\eta_{2}}=-\left[y_{\zeta_{4}}, y_{\xi_{16}}\right], \quad y_{\eta_{3}}=-\left[y_{\zeta_{3}}, y_{\xi_{16}}\right], \quad y_{\eta_{4}}=\left[y_{\zeta_{3}}, y_{\xi_{15}}\right],
$$




$$
\begin{aligned}
& y_{\eta_{5}}=-\left[y_{\zeta_{2}}, y_{\xi_{16}}\right], \quad y_{\eta_{6}}=\left[y_{\zeta_{2}}, y_{\xi_{15}}\right], \quad y_{\eta_{7}}=-\left[y_{\zeta_{1}}, y_{\xi_{16}}\right], \quad y_{\eta_{8}}=\left[y_{\zeta_{1}}, y_{\xi_{15}}\right], \\
& y_{\eta_{9}}=-\left[y_{\zeta_{2}}, y_{\xi_{14}}\right], \quad y_{\eta_{10}}=-\left[y_{\zeta_{1}}, y_{\xi_{14}}\right], \quad y_{\eta_{11}}=\left[y_{\zeta_{2}}, y_{\xi_{13}}\right], \quad y_{\eta_{12}}=\left[y_{\zeta_{1}}, y_{\xi_{13}}\right] \text {, } \\
& y_{\eta_{13}}=\left[y_{\zeta_{1}}, y_{\xi_{12}}\right], \quad y_{\eta_{14}}=-\left[y_{\zeta_{1}}, y_{\xi_{10}}\right], \quad y_{\eta_{15}}=\left[y_{\zeta_{1}}, y_{\xi_{8}}\right], \quad y_{\eta_{16}}=-\left[y_{\zeta_{1}}, y_{\xi_{7}}\right] \text {. }
\end{aligned}
$$

Finally, for $\theta \in \Phi_{3}^{+}$, we fix the orientation by the following;

$$
y_{\theta}=\left[y_{\eta_{16}}, y_{\xi_{16}}\right]
$$

Then, for example, we calculate

$$
\left[y_{\eta_{16}}, y_{\xi_{16}}\right]=\left[-\left[y_{\zeta_{1}}, y_{\xi_{7}}\right], y_{\xi_{16}}\right]=\left[-\left[y_{\zeta_{1}}, y_{\xi_{16}}\right], y_{\xi_{7}}\right]=\left[y_{\eta_{7}}, y_{\xi_{7}}\right]
$$

and obtain

$$
\left[y_{\eta_{p}}, y_{\xi_{q}}\right]=\delta_{p q} y_{\theta}, \quad\left[y_{\eta_{p}}, y_{\eta_{q}}\right]=\left[y_{\xi_{p}}, y_{\xi_{q}}\right]=0 \quad \text { for } \quad 1 \leqq p, q \leqq 16
$$

Moreover we calculate as in

$$
\left[y_{\zeta_{3}}, y_{\xi_{12}}\right]=\left[\left[y_{5}, y_{\zeta_{2}}\right], y_{\xi_{12}}\right]=\left[\left[y_{5}, y_{\xi_{12}}\right], y_{\zeta_{2}}\right]=\left[y_{\zeta_{2}},\left[y_{\xi_{12}}, y_{5}\right]\right]=\left[y_{\zeta_{2}}, y_{\xi_{14}}\right]=-y_{\eta_{9}},
$$

and obtain

$$
\begin{aligned}
& y_{\theta}=-\left[\left[y_{\zeta_{1}}, y_{\xi_{7}}\right], y_{\xi_{16}}\right]=\left[\left[y_{\zeta_{1}}, y_{\xi_{8}}\right], y_{\xi_{15}}\right]=-\left[\left[y_{\zeta_{1}}, y_{\xi_{10}}\right], y_{\xi_{14}}\right]=\left[\left[y_{\zeta_{1}}, y_{\xi_{12}}\right], y_{\xi_{13}}\right] \text {, } \\
& y_{\theta}=-\left[\left[y_{\zeta_{2}}, y_{\xi_{5}}\right], y_{\xi_{16}}\right]=\left[\left[y_{\zeta_{2}}, y_{\xi_{6}}\right], y_{\xi_{15}}\right]=-\left[\left[y_{\zeta_{2}}, y_{\xi_{9}}\right], y_{\xi_{14}}\right]=\left[\left[y_{\zeta_{2}}, y_{\xi_{11}}\right], y_{\xi_{13}}\right] \text {, } \\
& y_{\theta}=-\left[\left[y_{\zeta_{3}}, y_{\xi_{3}}\right], y_{\xi_{16}}\right]=\left[\left[y_{\zeta_{3}}, y_{\xi_{4}}\right], y_{\xi_{15}}\right]=-\left[\left[y_{\zeta_{3}}, y_{\xi_{9}}\right], y_{\xi_{12}}\right]=\left[\left[y_{\zeta_{3}}, y_{\xi_{10}}\right], y_{\xi_{11}}\right] \text {, } \\
& y_{\theta}=-\left[\left[y_{\zeta_{4}}, y_{\xi_{2}}\right], y_{\xi_{16}}\right]=\left[\left[y_{\zeta_{4}}, y_{\xi_{4}}\right], y_{\xi_{14}}\right]=-\left[\left[y_{\zeta_{4}}, y_{\xi_{6}}\right], y_{\xi_{12}}\right]=\left[\left[y_{\zeta_{4}}, y_{\xi_{8}}\right], y_{\xi_{11}}\right] \\
& y_{\theta}=-\left[\left[y_{\zeta_{5}}, y_{\xi_{1}}\right], y_{\xi_{16}}\right]=\left[\left[y_{\zeta_{5}}, y_{\xi_{4}}\right], y_{\xi_{13}}\right]=-\left[\left[y_{\zeta_{5}}, y_{\xi_{6}}\right], y_{\xi_{10}}\right]=\left[\left[y_{\zeta_{5}}, y_{\xi_{8}}\right], y_{\xi_{9}}\right] \text {, } \\
& y_{\theta}=-\left[\left[y_{\zeta_{6}}, y_{\xi_{2}}\right], y_{\xi_{15}}\right]=\left[\left[y_{\zeta_{6}}, y_{\xi_{3}}\right], y_{\xi_{14}}\right]=-\left[\left[y_{\zeta_{6}}, y_{\xi_{5}}\right], y_{\xi_{12}}\right]=\left[\left[y_{\zeta_{6}}, y_{\xi_{7}}\right], y_{\xi_{11}}\right] \text {, } \\
& y_{\theta}=-\left[\left[y_{\zeta_{7}}, y_{\xi_{1}}\right], y_{\xi_{15}}\right]=\left[\left[y_{\zeta_{7}}, y_{\xi_{3}}\right], y_{\xi_{13}}\right]=-\left[\left[y_{\zeta_{7}}, y_{\xi_{5}}\right], y_{\xi_{10}}\right]=\left[\left[y_{\zeta_{7}}, y_{\xi_{7}}\right], y_{\xi_{9}}\right] \text {, } \\
& y_{\theta}=-\left[\left[y_{\zeta_{8}}, y_{\xi_{1}}\right], y_{\xi_{14}}\right]=\left[\left[y_{\zeta_{8}}, y_{\xi_{2}}\right], y_{\xi_{13}}\right]=-\left[\left[y_{\zeta_{8}}, y_{\xi_{5}}\right], y_{\xi_{8}}\right]=\left[\left[y_{\zeta_{8}}, y_{\xi_{6}}\right], y_{\xi_{7}}\right] \text {, } \\
& y_{\theta}=-\left[\left[y_{\zeta_{9}}, y_{\xi_{1}}\right], y_{\xi_{12}}\right]=\left[\left[y_{\zeta_{9}}, y_{\xi_{2}}\right], y_{\xi_{10}}\right]=-\left[\left[y_{\zeta_{9}}, y_{\xi_{3}}\right], y_{\xi_{8}}\right]=\left[\left[y_{\zeta_{9}}, y_{\xi_{4}}\right], y_{\xi_{7}}\right] \\
& y_{\theta}=-\left[\left[y_{\zeta_{10}}, y_{\xi_{1}}\right], y_{\xi_{11}}\right]=\left[\left[y_{\zeta_{10}}, y_{\xi_{2}}\right], y_{\xi_{9}}\right]=-\left[\left[y_{\zeta_{10}}, y_{\xi_{3}}\right], y_{\xi_{6}}\right]=\left[\left[y_{\zeta_{10}}, y_{\xi_{4}}\right], y_{\xi_{5}}\right] \text {. }
\end{aligned}
$$

From (4.18), we have $S_{-2}=V^{*}$, by fixing the base of $S_{-3} \cong \mathbb{K}$ and putting $\mathfrak{l}_{-1}=V$. Moreover, from (4.19), $S_{-1}$ is embedded as the 10-dimensional subspace of $S^{2}\left(V^{*}\right)$ spanned by the following quadratic forms $f_{1}, \ldots, f_{10}$;

$$
\begin{array}{ll}
f_{1}(X)=-x_{7} x_{16}+x_{8} x_{15}-x_{10} x_{14}+x_{12} x_{13}, & f_{2}(X)=-x_{5} x_{16}+x_{6} x_{15}-x_{9} x_{14}+x_{11} x_{13}, \\
f_{3}(X)=-x_{3} x_{16}+x_{4} x_{15}-x_{9} x_{12}+x_{10} x_{11}, & f_{4}(X)=-x_{2} x_{16}+x_{4} x_{14}-x_{6} x_{12}+x_{8} x_{11}, \\
f_{5}(X)=-x_{1} x_{16}+x_{4} x_{13}-x_{6} x_{10}+x_{8} x_{9}, & f_{6}(X)=-x_{2} x_{15}+x_{3} x_{14}-x_{5} x_{12}+x_{7} x_{11}, \\
f_{7}(X)=-x_{1} x_{15}+x_{3} x_{13}-x_{5} x_{10}+x_{7} x_{9}, & f_{8}(X)=-x_{1} x_{14}+x_{2} x_{13}-x_{5} x_{8}+x_{6} x_{7}, \\
f_{9}(X)=-x_{1} x_{12}+x_{2} x_{10}-x_{3} x_{8}+x_{4} x_{7}, & f_{10}(X)=-x_{1} x_{11}+x_{2} x_{9}-x_{3} x_{6}+x_{4} x_{5} .
\end{array}
$$

for $X=\sum_{i=1}^{16} x_{i} y_{\xi_{i}} \in \mathfrak{l}_{-1}$. Thus, by fixing the basis $\left\{y_{\theta}\right\}$ of $S_{-3}$ and $\left\{y_{\xi_{1}}, \ldots, y_{\xi_{16}}\right\}$ of $\mathfrak{l}_{-1}$, an element $A=\sum_{i=1}^{10} a_{i} \operatorname{ad}\left(y_{\zeta_{i}}\right) \in S_{-1} \subset S^{2}\left(V^{*}\right) \cong \operatorname{Sym}(16)$ is represented as the 
symmetric matix of the following form;

$$
\left(\begin{array}{cccccccccccccccc}
0 & 0 & 0 & 0 & 0 & 0 & 0 & 0 & 0 & 0 & a_{10} & a_{9} & 0 & a_{8} & a_{7} & a_{5} \\
0 & 0 & 0 & 0 & 0 & 0 & 0 & 0 & a_{10}{ }^{*} & a_{9}{ }^{*} & 0 & 0 & a_{8}{ }^{*} & 0 & a_{6} & a_{4} \\
0 & 0 & 0 & 0 & 0 & a_{10} & 0 & a_{9} & 0 & 0 & 0 & 0 & a_{7}{ }^{*} & a_{6}{ }^{*} & 0 & a_{3} \\
0 & 0 & 0 & 0 & a_{10}{ }^{*} & 0 & a_{9}{ }^{*} & 0 & 0 & 0 & 0 & 0 & a_{5}{ }^{*} & a_{4}{ }^{*} & a_{3}{ }^{*} & 0 \\
0 & 0 & 0 & a_{10}{ }^{*} & 0 & 0 & 0 & a_{8} & 0 & a_{7} & 0 & a_{6} & 0 & 0 & 0 & a_{2} \\
0 & 0 & a_{10} & 0 & 0 & 0 & a_{8}{ }^{*} & 0 & 0 & a_{5} & 0 & a_{4} & 0 & 0 & a_{2}{ }^{*} & 0 \\
0 & 0 & 0 & a_{9}{ }^{*} & 0 & a_{8}{ }^{*} & 0 & 0 & a_{7}{ }^{*} & 0 & a_{6}{ }^{*} & 0 & 0 & 0 & 0 & a_{1} \\
0 & 0 & a_{9} & 0 & a_{8} & 0 & 0 & 0 & a_{5}{ }^{*} & 0 & a_{4}{ }^{*} & 0 & 0 & 0 & a_{1}{ }^{*} & 0 \\
0 & a_{10}{ }^{*} & 0 & 0 & 0 & 0 & a_{7}{ }^{*} & a_{5}{ }^{*} & 0 & 0 & 0 & a_{3} & 0 & a_{2} & 0 & 0 \\
0 & a_{9}{ }^{*} & 0 & 0 & a_{7} & a_{5} & 0 & 0 & 0 & 0 & a_{3}{ }^{*} & 0 & 0 & a_{1} & 0 & 0 \\
a_{10} & 0 & 0 & 0 & 0 & 0 & a_{6}{ }^{*} & a_{4}{ }^{*} & 0 & a_{3}{ }^{*} & 0 & 0 & a_{2}{ }^{*} & 0 & 0 & 0 \\
a_{9} & 0 & 0 & 0 & a_{6} & a_{4} & 0 & 0 & a_{3} & 0 & 0 & 0 & a_{1}{ }^{*} & 0 & 0 & 0 \\
0 & a_{8}{ }^{*} & a_{7}{ }^{*} & a_{5}{ }^{*} & 0 & 0 & 0 & 0 & 0 & 0 & a_{2}{ }^{*} & a_{1}{ }^{*} & 0 & 0 & 0 & 0 \\
a_{8} & 0 & a_{6}{ }^{*} & a_{4}{ }^{*} & 0 & 0 & 0 & 0 & a_{2} & a_{1} & 0 & 0 & 0 & 0 & 0 & 0 \\
a_{7} & a_{6} & 0 & a_{3}{ }^{*} & 0 & a_{2}{ }^{*} & 0 & a_{1}{ }^{*} & 0 & 0 & 0 & 0 & 0 & 0 & 0 & 0 \\
a_{5} & a_{4} & a_{3} & 0 & a_{2} & 0 & a_{1} & 0 & 0 & 0 & 0 & 0 & 0 & 0 & 0 & 0
\end{array}\right)
$$

where $a_{i}{ }^{*}=-a_{i}$. Hence the standard differential system $\left(M(\mathfrak{m}), D_{\mathfrak{m}}\right)$ of type $\mathfrak{m}$ in this case is given by

$$
D_{\mathfrak{m}}=\left\{\varpi=\varpi_{1}=\varpi_{2}=\cdots=\varpi_{16}=0\right\}
$$

where

$$
\begin{aligned}
\varpi & =d y-p_{1} d x_{1}-\cdots-p_{16} d x_{16}, \\
\varpi_{1} & =d p_{1}+q_{10} d x_{11}+q_{9} d x_{12}+q_{8} d x_{14}+q_{7} d x_{15}+q_{5} d x_{16}, \\
\varpi_{2} & =d p_{2}-q_{10} d x_{9}-q_{9} d x_{10}-q_{8} d x_{13}+q_{6} d x_{15}+q_{4} d x_{16}, \\
\varpi_{3} & =d p_{3}+q_{10} d x_{6}+q_{9} d x_{8}-q_{7} d x_{13}-q_{6} d x_{14}+q_{3} d x_{16}, \\
\varpi_{4} & =d p_{4}-q_{10} d x_{5}-q_{9} d x_{7}-q_{5} d x_{13}-q_{4} d x_{14}-q_{3} d x_{15}, \\
\varpi_{5} & =d p_{5}-q_{10} d x_{4}+q_{8} d x_{8}+q_{7} d x_{10}+q_{6} d x_{12}+q_{2} d x_{16}, \\
\varpi_{6} & =d p_{6}+q_{10} d x_{3}-q_{8} d x_{7}+q_{5} d x_{10}+q_{4} d x_{12}-q_{2} d x_{15}, \\
\varpi_{7} & =d p_{7}-q_{9} d x_{4}-q_{8} d x_{6}-q_{7} d x_{9}-q_{6} d x_{11}+q_{1} d x_{16}, \\
\varpi_{8} & =d p_{8}+q_{9} d x_{3}+q_{8} d x_{5}-q_{5} d x_{9}-q_{4} d x_{11}-q_{1} d x_{15}, \\
\varpi_{9} & =d p_{9}-q_{10} d x_{2}-q_{7} d x_{7}-q_{5} d x_{8}+q_{3} d x_{12}+q_{2} d x_{14}, \\
\varpi_{10} & =d p_{10}-q_{9} d x_{2}+q_{7} d x_{5}+q_{5} d x_{6}-q_{3} d x_{11}+q_{1} d x_{14}, \\
\varpi_{11} & =d p_{11}+q_{10} d x_{1}-q_{6} d x_{7}-q_{4} d x_{8}-q_{3} d x_{10}-q_{2} d x_{13}, \\
\varpi_{12} & =d p_{12}+q_{9} d x_{1}+q_{6} d x_{5}+q_{4} d x_{6}+q_{3} d x_{9}-q_{1} d x_{13}, \\
\varpi_{13} & =d p_{13}-q_{8} d x_{2}-q_{7} d x_{3}-q_{5} d x_{4}-q_{2} d x_{11}-q_{1} d x_{12}, \\
\varpi_{14} & =d p_{14}+q_{8} d x_{1}-q_{6} d x_{3}-q_{4} d x_{4}+q_{2} d x_{9}+q_{1} d x_{10}, \\
\varpi_{15} & =d p_{15}+q_{7} d x_{1}+q_{6} d x_{2}-q_{3} d x_{4}-q_{2} d x_{6}-q_{1} d x_{8}, \\
\varpi_{16} & =d p_{16}+q_{5} d x_{1}+q_{4} d x_{2}+q_{3} d x_{3}+q_{2} d x_{5}+q_{1} d x_{7} .
\end{aligned}
$$

Here $\left(x_{1}, \ldots, x_{16}, y, p_{1}, \ldots, p_{16}, q_{1}, \ldots, q_{10}\right)$ is a coordinate system of $M(\mathfrak{m}) \cong \mathbb{K}^{43}$. Thus the model equation of our typical symbol $\mathfrak{m}=\check{\mathfrak{g}}_{-3} \oplus \check{\mathfrak{g}}_{-2} \oplus \check{\mathfrak{g}}_{-1} \subset \mathfrak{C}^{2}\left(\mathfrak{l}_{-1}, \mathbb{K}\right)$ is given by

$$
\frac{\partial^{2} y}{\partial x_{7} \partial x_{16}}=-\frac{\partial^{2} y}{\partial x_{8} \partial x_{15}}=\frac{\partial^{2} y}{\partial x_{10} \partial x_{14}}=-\frac{\partial^{2} y}{\partial x_{12} \partial x_{13}}
$$




$$
\begin{aligned}
& \frac{\partial^{2} y}{\partial x_{5} \partial x_{16}}=-\frac{\partial^{2} y}{\partial x_{6} \partial x_{15}}=\frac{\partial^{2} y}{\partial x_{9} \partial x_{14}}=-\frac{\partial^{2} y}{\partial x_{11} \partial x_{13}} \\
& \frac{\partial^{2} y}{\partial x_{3} \partial x_{16}}=-\frac{\partial^{2} y}{\partial x_{4} \partial x_{15}}=\frac{\partial^{2} y}{\partial x_{9} \partial x_{12}}=-\frac{\partial^{2} y}{\partial x_{10} \partial x_{11}} \\
& \frac{\partial^{2} y}{\partial x_{2} \partial x_{16}}=-\frac{\partial^{2} y}{\partial x_{4} \partial x_{14}}=\frac{\partial^{2} y}{\partial x_{6} \partial x_{12}}=-\frac{\partial^{2} y}{\partial x_{8} \partial x_{11}} \\
& \frac{\partial^{2} y}{\partial x_{1} \partial x_{16}}=-\frac{\partial^{2} y}{\partial x_{4} \partial x_{13}}=\frac{\partial^{2} y}{\partial x_{6} \partial x_{10}}=-\frac{\partial^{2} y}{\partial x_{8} \partial x_{9}} \\
& \frac{\partial^{2} y}{\partial x_{2} \partial x_{15}}=-\frac{\partial^{2} y}{\partial x_{3} \partial x_{14}}=\frac{\partial^{2} y}{\partial x_{5} \partial x_{12}}=-\frac{\partial^{2} y}{\partial x_{7} \partial x_{11}} \\
& \frac{\partial^{2} y}{\partial x_{1} \partial x_{15}}=-\frac{\partial^{2} y}{\partial x_{3} \partial x_{13}}=\frac{\partial^{2} y}{\partial x_{5} \partial x_{10}}=-\frac{\partial^{2} y}{\partial x_{7} \partial x_{9}} \\
& \frac{\partial^{2} y}{\partial x_{1} \partial x_{14}}=-\frac{\partial^{2} y}{\partial x_{2} \partial x_{13}}=\frac{\partial^{2} y}{\partial x_{5} \partial x_{8}}=-\frac{\partial^{2} y}{\partial x_{6} \partial x_{7}} \\
& \frac{\partial^{2} y}{\partial x_{1} \partial x_{12}}=-\frac{\partial^{2} y}{\partial x_{2} \partial x_{10}}=\frac{\partial^{2} y}{\partial x_{3} \partial x_{8}}=-\frac{\partial^{2} y}{\partial x_{4} \partial x_{7}} \\
& \frac{\partial^{2} y}{\partial x_{1} \partial x_{11}}=-\frac{\partial^{2} y}{\partial x_{2} \partial x_{9}}=\frac{\partial^{2} y}{\partial x_{3} \partial x_{6}}=-\frac{\partial^{2} y}{\partial x_{4} \partial x_{5}} \\
& \frac{\partial^{2} y}{\partial x_{i} \partial x_{j}}=0 \\
& \text { otherwise. }
\end{aligned}
$$

where $y$ is dependent variable and $x_{1}, \ldots, x_{16}$ are independent variables. By a direct calculation, we see that the prolongation of the second order system (4.20) is given by

$$
\frac{\partial^{3} y}{\partial x_{i} \partial x_{j} \partial x_{k}}=0 \quad \text { for } \quad 1 \leqq i, j, k \leqq 16 .
$$

\section{Equivalence of Parabolic Geometries}

In this section, we will discuss about the equivalence of each parabolic geometry associated with the differential equations of finite type explicitly described in $\S 3$ and $\S 4$.

In the following, we will first show a common property of the typical symbol $\mathfrak{m}$ of type $(\mathfrak{l}, S)$. Here $\mathfrak{m}=\bigoplus_{p=-1}^{-\mu} \mathfrak{g}_{p}$ is a graded subalgebra of $\mathfrak{C}^{\mu-1}(V, W)$, which has the splitting $\mathfrak{g}_{-1}=\mathfrak{l}_{-1} \oplus S_{-1}$, where $V=\mathfrak{l}_{-1}$ and $W=S_{-\mu}$. In particular $S_{p} \subset W \otimes S^{\mu+p}\left(V^{*}\right)$. Thus we have the notion of the algebraic prolongation $\rho\left(S_{p}\right)$ of $S_{p}$, which is defined by

$$
\rho\left(S_{p}\right)=S_{p} \otimes V^{*} \cap W \otimes S^{\mu+p+1}\left(V^{*}\right) \quad \text { for } \quad-\mu+1 \leqq p \leqq-1 .
$$

We will show the following Proposition 5.1 concerning the property of the prolongations of $S_{p}$ for the typical symbol $\mathfrak{m}$ of type $(\mathfrak{l}, S)$.

Let $\mathfrak{g}=\bigoplus_{p=-\mu}^{1} \mathfrak{g}_{p}$ be a pseudo-product GLA of type $(\mathfrak{l}, S)$.

Lemma 5.1. Let $p$ be an integer with $-\mu+1 \leqq p \leqq-1$. If $H^{1}(\mathfrak{m}, \mathfrak{g})_{p+1,-1}=0$, then the algebraic prolongation $\rho\left(S_{p}\right)$ of $S_{p}$ is equal to $S_{p+1}$, where we put $S_{0}=0$.

Proof. Since the fact $S_{p+1} \subset \rho\left(S_{p}\right)$ is clear, it is sufficient to prove that $\rho\left(S_{p}\right) \subset S_{p+1}$. Let $\varphi$ be an element of $\rho\left(S_{p}\right)$. The space $\rho\left(S_{p}\right)$ can be considered as a subspace of $\operatorname{Hom}\left(\mathfrak{l}_{-1}, S_{p}\right)$. 
We define an element $\tilde{\varphi}$ of $C^{1}(\mathfrak{m}, \mathfrak{g})_{p+1,-1}$ as follows:

$$
\tilde{\varphi}(X)=\varphi(X)\left(X \in \mathfrak{l}_{-1}\right), \quad \tilde{\varphi}(S)=0 .
$$

Then we have

$$
\partial \tilde{\varphi}\left(X_{1}, X_{2}\right)=\left[X_{1}, \varphi\left(X_{2}\right)\right]-\left[X_{2}, \varphi\left(X_{1}\right)\right] \text { for } X_{1}, X_{2} \in \mathfrak{l}_{-1} .
$$

Since $\varphi \in \rho\left(S_{p}\right)$, we get $\partial \tilde{\varphi}=0$. Also since $H^{1}(\mathfrak{m}, \mathfrak{g})_{p+1,-1}=0$, there exits an element $s \in S_{p+1}$ such that $\partial s=\tilde{\varphi}$. Hence $\rho\left(S_{p}\right) \subset S_{p+1}$.

For a pseudo-product GLA $\mathfrak{g}$ of type $(\mathfrak{l}, S)$, we furthermore assume that the prolongation $\check{\mathfrak{g}}=\bigoplus_{p \in \mathbb{Z}} \check{\mathfrak{g}}_{p}$ of $\left(\mathfrak{m}, \mathfrak{g}_{0}\right)$ is a simple graded Lie algebra (SGLA), where $\mathfrak{m}=\mathfrak{g}_{-}$.

Now we investigate the space $H^{1}(\mathfrak{m}, \mathfrak{g})_{r,-1}$. Note that, from $\check{\mathfrak{g}}_{p}=\mathfrak{g}_{p}$ for $p \leqq 0$, $H^{1}(\mathfrak{m}, \mathfrak{g})_{r,-1}=H^{1}(\mathfrak{m}, \check{\mathfrak{g}})_{r,-1}$ for $r \leqq 1$. Also we know that $H^{1}(\mathfrak{m}, \mathfrak{g})_{r,-1}$ is isomorphic to $H^{1}\left(\mathfrak{l}_{-1}, S\right)_{r}$ as a $\mathfrak{g}_{0}$-module(see $\S 5$ in [YY02]). Let $\Sigma=\left\{\gamma_{1}, \ldots, \gamma_{L}\right\}$ be a simple root system of $\check{\mathfrak{g}}$ and let $\theta$ be the highest root of $\check{\mathfrak{g}}$. Assume that

(i) The SGLA $\check{\mathfrak{g}}$ is of type $\left(Y_{L},\left\{\gamma_{a}, \gamma_{b}\right\}\right)$;

(ii) $\mathfrak{l}_{-1}$ is a $\mathfrak{g}_{0}$-module with highest weight $-\gamma_{a}$;

(iii) $S_{-1}$ is a $\mathfrak{g}_{0}$-module with highest weight $-\gamma_{b}$.

By Kostant's theorem, $H^{1}(\mathfrak{m}, \mathfrak{g})_{r,-1}$ is an irreducible $\mathfrak{g}_{0}$-module with lowest weight $\sigma_{a}(-\theta-$ $\delta)+\delta$, where we use the notations in [Yam93]. Let $E$ be the characteristic element of the GLA $\mathfrak{\mathfrak { g }}$; then

$$
\left(\sigma_{a}(-\theta-\delta)+\delta\right)(E)=-\mu+\left\langle\theta, \gamma_{a}\right\rangle+1
$$

Hence $H^{1}(\mathfrak{m}, \check{\mathfrak{g}})_{r,-1} \neq 0$ if and only if $r=-\mu+\left\langle\theta, \gamma_{a}\right\rangle+1$. From the table in Theorem 2.1 (a) and [Bou68], we obtain the following lemma.

Lemma 5.2. Under the above assumptions, we have

(1) Assume that $\left(Y_{L},\left\{\gamma_{a}, \gamma_{b}\right\}\right)$ is one of the following types: $\left(A_{\ell+n+1},\left\{\gamma_{1}, \gamma_{\ell+1}\right\}\right)(n \geqq$ $0, \ell \geqq 1),\left(C_{\ell+1},\left\{\gamma_{1}, \gamma_{\ell+1}\right\}\right)(\ell \geqq 1)$. Then $H^{1}(\mathfrak{m}, \mathfrak{g})_{r,-1} \neq 0$ if and only if $r=0$.

(2) Assume that $\left(Y_{L},\left\{\gamma_{a}, \gamma_{b}\right\}\right)$ is one of the following types: $\left(A_{\ell+n+1},\left\{\gamma_{i}, \gamma_{\ell+1}\right\}\right)(1<$ $i \leqq \ell, \ell \geqq 2, n \geqq 0),\left(B_{\ell+1},\left\{\gamma_{2}, \gamma_{1}\right\}\right)(\ell \geqq 2),\left(D_{\ell+1},\left\{\gamma_{2}, \gamma_{1}\right\}\right)(\ell \geqq 4),\left(D_{\ell+1},\left\{\gamma_{\ell+1}, \gamma_{1}\right\}\right)$ $(\ell \geqq 4),\left(D_{\ell+1},\left\{\gamma_{1}, \gamma_{\ell+1}\right\}\right)(\ell \geqq 3),\left(D_{\ell+1},\left\{\gamma_{\ell}, \gamma_{\ell+1}\right\}\right)(\ell \geqq 3),\left(D_{\ell+1},\left\{\gamma_{2}, \gamma_{\ell+1}\right\}\right)$ $(\ell \geqq 3),\left(E_{6},\left\{\gamma_{1}, \gamma_{6}\right\}\right),\left(E_{6},\left\{\gamma_{2}, \gamma_{1}\right\}\right),\left(E_{7},\left\{\gamma_{1}, \gamma_{7}\right\}\right)$. Then $H^{1}(\mathfrak{m}, \mathfrak{g})_{r,-1} \neq 0$ if and only if $r=-1$.

(3) Assume that $\left(Y_{L},\left\{\gamma_{a}, \gamma_{b}\right\}\right)$ is one of the following types: $\left(C_{\ell+1},\left\{\gamma_{i}, \gamma_{\ell+1}\right\}\right)(1<$ $i \leqq \ell, \ell \geqq 2),\left(D_{\ell+1},\left\{\gamma_{i}, \gamma_{\ell+1}\right\}\right)(2<i<\ell, \ell \geqq 4),\left(E_{6},\left\{\gamma_{3}, \gamma_{1}\right\}\right),\left(E_{7},\left\{\gamma_{6}, \gamma_{7}\right\}\right)$. Then $H^{1}(\mathfrak{m}, \mathfrak{g})_{r,-1} \neq 0$ if and only if $r=-2$.

By Lemmas 5.1 and 5.2, we get the following proposition.

Proposition 5.1. Under the above assumptions, we have

(1) Unless $\left(Y_{L},\left\{\gamma_{a}, \gamma_{b}\right\}\right)$ is $\left(A_{\ell+n+1},\left\{\gamma_{1}, \gamma_{\ell+1}\right\}\right)(n \geqq 0, \ell \geqq 1)$ or $\left(C_{\ell+1},\left\{\gamma_{1}, \gamma_{\ell+1}\right\}\right)$ $(\ell \geqq 1)$, the algebraic prolongation $\rho\left(S_{-1}\right)$ of $S_{-1}$ is $\{0\}$.

(2) Assume that $\left(Y_{L},\left\{\gamma_{a}, \gamma_{b}\right\}\right)$ is one of the following types: $\left(C_{\ell+1},\left\{\gamma_{i}, \gamma_{\ell+1}\right\}\right)(1<$ $i \leqq \ell, \ell \geqq 2),\left(D_{\ell+1},\left\{\gamma_{i}, \gamma_{\ell+1}\right\}\right)(2<i<\ell, \ell \geqq 4),\left(E_{6},\left\{\gamma_{3}, \gamma_{1}\right\}\right),\left(E_{7},\left\{\gamma_{6}, \gamma_{7}\right\}\right)$. Then $\rho\left(S_{-2}\right)=S_{-1}$ and $\rho\left(S_{-1}\right)=0$.

Actually we can check these properties by direct calculations in each cases in the previous sections. By these properties of the typical symbols, we can classify our parabolic geometries into the following four groups. 
(A) The parabolic geometry associated with $\left(A_{\ell+n+1},\left\{\gamma_{1}, \gamma_{\ell+1}\right\}\right)(n \geqq 0, \ell \geqq 1)$ is the geometry of the pseudo-pojective systems of second order of bidegree $(\ell, n+1)$, i.e. the geometry of the second order equations of $\ell$ independent and $n+1$ dependent variables by point transformations. The parabolic geometry associated with $\left(C_{\ell+1},\left\{\gamma_{1}, \gamma_{\ell+1}\right\}\right)(\ell \geqq 1)$ is the geometry of the pseudo-projective systems of third order of bidegree $(\ell, 1)$, i.e. the geometry of the third order equations of $\ell$ independent and 1 dependent variables by contact transformations.

(B) The parabolic geometries associated with $\left(A_{\ell+n+1},\left\{\gamma_{i}, \gamma_{\ell+1}\right\}\right)(2 \leqq i \leqq \ell, n \geqq$ $0),\left(D_{\ell+1},\left\{\gamma_{\ell+1}, \gamma_{1}\right\}\right)(\ell \geqq 4),\left(D_{\ell+1},\left\{\gamma_{1}, \gamma_{\ell+1}\right\}\right)(\ell \geqq 3),\left(D_{\ell+1},\left\{\gamma_{\ell}, \gamma_{\ell+1}\right\}\right)(\ell \geqq 3)$ and $\left(E_{6},\left\{\gamma_{1}, \gamma_{6}\right\}\right)$ are the contact geometries of finite type equations of the first order in the following sense.

In this case $\mu=2$ and the typical symbol $\mathfrak{m}$ has the following description: $\mathfrak{m}=$ $\mathfrak{g}_{-2} \oplus \mathfrak{g}_{-1} \subset \mathfrak{C}^{1}(V, W)$, where $W=S_{-2}$ and $V=\mathfrak{l}_{-1}$. Moreover $\mathfrak{g}_{-1}=V \oplus S_{-1}$ and $S_{-1} \subset W \otimes V^{*}$. Let $J^{k}(n, m)$ be the space of $k$-jets of $n$ independent and $m$ dependent variables, where $n=\operatorname{dim} V$ and $m=\operatorname{dim} W$. We consider a submanifold $R$ of $J^{1}(n, m)$ such that $\left.\pi_{0}^{1}\right|_{R}: R \rightarrow J^{0}(n, m)$ is a submersion. Let $D$ be the restriction to $R$ of the canonical systetem $C^{1}$ on $J^{1}(n, m)$ and $R^{(1)} \subset J^{2}(n, m)$ be the first prolongation of $R$ (cf. $\S 4.2$ [Yam82]). We assume that $p^{(1)}: R^{(1)} \rightarrow R$ is onto. This assumption is equivalent to say that $(R, D)$ has an (n-dimensional) integral element (transversal to the fibre $\left.\operatorname{Ker}\left(\pi_{0}^{1}||_{R}\right)_{*}\right)$ at each point of $R$. Under this integrability condition, $(R, D)$ is a regular differential system of type $\mathfrak{m}$ if and only if the symbols of this equation $R$ are isomorphic to $S_{-1} \subset W \otimes V^{*}$ at each point of $R$ (see $\S 2.1$ in [SYY97] for the precise meaning of the isomorphism of the symbol). In this case, by (1) of Proposition 5.1, integral elements of $(R, D)$ are unique at each point of $R$ so that $p^{(1)}: R^{(1)} \rightarrow R$ is a diffeomorphism. Thus $(R, D)$ has the (almost) pseudo-product structure corresponding to the splitting $\mathfrak{g}_{-1}=V \oplus S_{-1}$. In fact $S_{-1}$ corresponds to the fibre direction $\operatorname{Ker}\left(\left.\pi_{0}^{1}\right|_{R}\right)_{*}$ and $V$ corresponds to the restriction to $R^{(1)}$ of the canonical system $C^{2}$ on $J^{2}(n, m)$. Since $\check{\mathfrak{g}}$ is the prolongation of $\mathfrak{m}$, an isomorphism of $(R, D)$ preserves the pseudo-product structure. In particular an isomorphism of $(R, D)$ preserves the projection $\left.\pi_{0}^{1}\right|_{R}: R \rightarrow J^{0}(n, m)$. Hence a local isomorphism of $(R, D)$ is the lift of a local point transformation of $J^{0}(n, m)$.

By Theorem 2.7 and 2.9 [Tan79] and Proposition 5.5 [Yam93], we observe that parabolic geometries associated with $\left(A_{\ell+n+1},\left\{\gamma_{i}, \gamma_{\ell+1}\right\}\right)(3 \leqq i \leqq \ell-1, n \geqq 2),\left(D_{\ell+1},\left\{\gamma_{\ell}, \gamma_{\ell+1}\right\}\right)$ $(\ell \geqq 3)$ and $\left(E_{6},\left\{\gamma_{1}, \gamma_{6}\right\}\right)$ have no local invariants. Hence in these cases, $(R, D)$, satisfying the integrability condition, is always locally isomorphic to the model equation given in Case (1), (8) of $\S 3$ or Case (1) of $\S 4$ respectively.

(C) The parabolic geometries associated with $\left(B_{\ell+1},\left\{\gamma_{2}, \gamma_{1}\right\}\right)(\ell \geqq 2),\left(D_{\ell+1},\left\{\gamma_{2}, \gamma_{1}\right\}\right)$ $(\ell \geqq 4),\left(D_{\ell+1},\left\{\gamma_{2}, \gamma_{\ell+1}\right\}\right)(\ell \geqq 3),\left(E_{6},\left\{\gamma_{2}, \gamma_{1}\right\}\right)$ and $\left(E_{7},\left\{\gamma_{1}, \gamma_{7}\right\}\right)$ are the contact geometries of finite type equations of the second order in the following sense.

In this case $\mu=3$ and the typical symbol $\mathfrak{m}$ has the following description: $\mathfrak{m}=$ $\mathfrak{g}_{-3} \oplus \mathfrak{g}_{-2} \oplus \mathfrak{g}_{-1} \subset \mathfrak{C}^{2}(V, W)$, where $W=\mathbb{K}, V=\mathfrak{l}_{-1}$ and $\operatorname{dim} V=n$. Moreover we have $\mathfrak{g}_{-2}=V^{*}, \mathfrak{g}_{-1}=V \oplus S_{-1}$ and $S_{-1} \subset S^{2}\left(V^{*}\right)$. In this case, we note that the standard differential system $\left(M_{\mathfrak{g}}, D_{\mathfrak{g}}\right)$ of type $\left(Y_{L},\left\{\gamma_{a}\right\}\right)$ is the standard contact manifold of type $Y_{L}$ (see $\S 4$ in [Yam93]).

We consider a submanifold $R$ of $J^{2}(n, 1)$ such that $\left.\pi_{1}^{2}\right|_{R}: R \rightarrow J^{1}(n, 1)$ is a submersion. Let $D$ be the restriction to $R$ of the canonical systetem $C^{2}$ on $J^{2}(n, 1)$ and $R^{(1)} \subset J^{3}(n, 1)$ be the first prolongation of $R$. We assume that $p^{(1)}: R^{(1)} \rightarrow R$ is onto. Under this integrability condition, $(R, D)$ is a regular differential system of type $\mathfrak{m}$ if and only if the symbols of this equation $R$ are isomorphic to $S_{-1} \subset S^{2}\left(V^{*}\right)$ at each point of $R$. In this 
case, by (1) of Proposition 5.1, integral elements of $(R, D)$ are unique at each point of $R$ so that $p^{(1)}: R^{(1)} \rightarrow R$ is a diffeomorphism. Thus $(R, D)$ has the (almost) pseudo-product structure corresponding to the splitting $\mathfrak{g}_{-1}=V \oplus S_{-1}$. In fact $S_{-1}$ corresponds to the fibre direction $\operatorname{Ker}\left(\left.\pi_{1}^{2}\right|_{R}\right)_{*}$ and $V$ corresponds to the restriction to $R^{(1)}$ of the canonical system $C^{3}$ on $J^{3}(n, 1)$. Since $\check{\mathfrak{g}}$ is the prolongation of $\mathfrak{m}$, an isomorphism of $(R, D)$ preserves the pseudo-product structure. In particular an isomorphism of $(R, D)$ preserves the projection $\left.\pi_{1}^{2}\right|_{R}: R \rightarrow J^{1}(n, 1)$ and $\partial D=\left(\pi_{1}^{2}\right)_{*}^{-1}\left(C^{1}\right)$. Hence a local isomorphism of $(R, D)$ is the lift of a local contact transformation of $J^{1}(n, 1)$.

By Theorem 2.7 and 2.9 [Tan79] and Proposition 5.5 [Yam93], we observe that parabolic geometries associated with $\left(D_{\ell+1},\left\{\gamma_{2}, \gamma_{\ell+1}\right\}\right)(\ell \geqq 3),\left(E_{6},\left\{\gamma_{2}, \gamma_{1}\right\}\right)$ and $\left(E_{7},\left\{\gamma_{1}, \gamma_{7}\right\}\right)$ have no local invariants. Hence in these cases, $(R, D)$, satisfying the integrability condition, is always locally isomorphic to the model equation given in Case (10) of $\S 3$ or Case (3), (5) of $\S 4$ respectively. The rigidity of the parabolic geometry associated with $\left(D_{\ell+1},\left\{\gamma_{2}, \gamma_{\ell+1}\right\}\right)$ $(\ell \geqq 3)$ is already discussed in [YY02] in connection with the Plücker embedding equations.

(D) The parabolic geometries associated with $\left(C_{\ell+1},\left\{\gamma_{i}, \gamma_{\ell+1}\right\}\right)(1<i \leqq \ell, \ell \geqq 2)$, $\left(D_{\ell+1},\left\{\gamma_{i}, \gamma_{\ell+1}\right\}\right)(2<i<\ell, \ell \geqq 4),\left(E_{6},\left\{\gamma_{3}, \gamma_{1}\right\}\right)$ and $\left(E_{7},\left\{\gamma_{6}, \gamma_{7}\right\}\right)$ are the geometries of finite type equations of the first order in the following sense.

In this case $\mu=3$ and the typical symbol $\mathfrak{m}$ has the following description: $\mathfrak{m}=\mathfrak{g}_{-3} \oplus$ $\mathfrak{g}_{-2} \oplus \mathfrak{g}_{-1} \subset \mathfrak{C}^{2}(V, W)$, where $W=S_{-3}$ and $V=\mathfrak{l}_{-1}$. Moreover $\mathfrak{g}_{-2}=S_{-2}, \mathfrak{g}_{-1}=V \oplus S_{-1}$, $S_{-2} \subset W \otimes V^{*}, S_{-1} \subset W \otimes S^{2}\left(V^{*}\right)$ and $\operatorname{dim} S_{-2}=\operatorname{dim} V$. In this case we first consider a submanifold $R$ of $J^{1}(n, m)$ such that $\left.\pi_{0}^{1}\right|_{R}: R \rightarrow J^{0}(n, m)$ is a submersion, where $n=\operatorname{dim} V$ and $m=\operatorname{dim} W$. Let $D$ be the restriction to $R$ of the canonical systetem $C^{1}$ on $J^{1}(n, m)$ and $R^{(1)} \subset J^{2}(n, m)$ be the first prolongation of $R$. We assume that the symbols of this equation $R$ are isomorphic to $S_{-2} \subset W \otimes V^{*}$ at each point of $R$ and also assume that $p^{(1)}: R^{(1)} \rightarrow R$ is onto. Then $(R, D)$ is a regular differential system of type $\hat{\mathfrak{m}}=\hat{\mathfrak{g}}_{-2} \oplus \hat{\mathfrak{g}}_{-1}$, where $\hat{\mathfrak{g}}_{-2}=W$ and $\hat{\mathfrak{g}}_{-1}=V \oplus S_{-2}$. Here the symbol algebra $\hat{\mathfrak{m}}$ is the negative part of the simple graded Lie algebra of type $\left(Y_{L},\left\{\gamma_{a}\right\}\right)$, i.e., of type $\left(C_{\ell+1},\left\{\gamma_{i}\right\}\right)(2 \leqq i \leqq \ell)$, $\left(D_{\ell+1},\left\{\gamma_{i}\right\}\right)(2<i<\ell),\left(E_{6},\left\{\gamma_{3}\right\}\right)$ and $\left(E_{7},\left\{\gamma_{6}\right\}\right)$ respectively. Furthermore, by $(2)$ of Proposition 5.1, the symbols of this equation $R^{(1)}$ are isomorphic to $\rho\left(S_{-2}\right)=S_{-1} \subset$ $W \otimes S^{2}\left(V^{*}\right)$. Let $D^{(1)}$ be the restriction to $R^{(1)}$ of the canonical system $C^{2}$ on $J^{2}(n, m)$ and $R^{(2)} \subset J^{3}(n, m)$ be the prolongation of $R^{(1)}$. We further assume that $p^{(2)}: R^{(2)} \rightarrow R^{(1)}$ is onto. Under these integrability conditions, $\left(R^{(1)}, D^{(1)}\right)$ becomes a regular differential system of type $\mathfrak{m}$. Actually the set of $n$-dimensional integral elements of $(R, D)$ forms a bundle over $R$, which contains $R^{(1)}$ as an open dense subset such that $D^{(1)}$ coincides with the canonical system induced by this Grassmanian construction (cf. $\S 2$ in [Yam82], $\S 1$ in [Yam99]). Moreover, by (1) of Proposition 5.1, integral elements of $\left(R^{(1)}, D^{(1)}\right)$ are unique at each point of $R^{(1)}$ so that $p^{(2)}: R^{(2)} \rightarrow R^{(1)}$ is a diffeomorphism. Thus $\left(R^{(1)}, D^{(1)}\right)$ has the (almost) pseudo-product structure corresponding to the splitting $\mathfrak{g}_{-1}=V \oplus$ $S_{-1}$. In fact $S_{-1}$ corresponds to the fibre direction $\operatorname{Ker}\left(p^{(1)}\right)_{*}$ and $V$ corresponds to the restriction to $R^{(2)}$ of the canonical system $C^{3}$ on $J^{3}(n, m)$. Since $\mathfrak{\mathfrak { g }}$ is the prolongation of $\mathfrak{m}$, an isomorphism of $\left(R^{(1)}, D^{(1)}\right)$ preserves the pseudo-product structure. In particular an isomorphism of $\left(R^{(1)}, D^{(1)}\right)$ preserves the projection $p^{(1)}: R^{(1)} \rightarrow R$ and $\partial D^{(1)}=$ $\left(p^{(1)}\right)_{*}^{-1}(D)$. Thus a local isomorphism of $\left(R^{(1)}, D^{(1)}\right)$ induces that of $(R, D)$ and coincides with the local lift of this isomorphism of $(R, D)$. Hence the local equivalence of $\left(R^{(1)}, D^{(1)}\right)$ is reducible to that of $(R, D)$.

By Theorem 2.7 and 2.9 [Tan79] and Proposition 5.5 [Yam93], we observe that parabolic geometries associated with $\left(C_{\ell+1},\left\{\gamma_{i}, \gamma_{\ell+1}\right\}\right)(2<i<\ell),\left(D_{\ell+1},\left\{\gamma_{i}, \gamma_{\ell+1}\right\}\right)(2<i<\ell)$, $\left(E_{6},\left\{\gamma_{3}, \gamma_{1}\right\}\right)$ and $\left(E_{7},\left\{\gamma_{6}, \gamma_{7}\right\}\right)$ have no local invariants. Hence in these cases, $(R, D)$, 
satisfying the integrability conditions, is always locally isomorphic to the model equation given in Case (3), (9) of $\S 3$ or Case (2), (4) of $\S 4$ respectively.

\section{REFERENCES}

[Bai93] T.N.Baily, Parabolic Invariant Theory and Geometry in "The Penrose Transform and Analytic Cohomology in Representation Theory" Contemp. Math. 154, Amer. Math. Soc., 1993.

[Bou68] N. Bourbaki, Groupes et algebres de Lie, Chapitres 4, 5 et 6, Hermann Paris (1968)

[Bou75] N. Bourbaki, Groupes et algebres de Lie, Chapitres 7 et 8, Hermann Paris (1975)

[BCG91] R. Bryant, S. Chern, R. Gardner, H. Goldschmidt and P. Griffiths : Exterior Differential Systems, MSRI Publ. vol. 18, Springer Verlag, Berlin 1991

[Car10] E. Cartan : Les systèmes de Pfaff à cinq variables et les équations aux dérivées partielles du second ordre, Ann. École Normale, 27 (1910), 109-192

[DKM99] B.Doubrov, B.Komrakov and T.Morimoto, Equivalence of holonomic differential equations, Lobachevskii J. of Math. 3 (1999), 39-71.

[Hum72] J.E.Humphreys, Introduction to Lie Algebras and Representation Theory, Springer-Verlag 1972.

[Kos61] B. Kostant, Lie algebra cohomology and generalized Borel-Weil theorem, Ann. of Math. 74 (1961), 329-397.

[Sea88] Y. Se-ashi, On differential invariants of integrable finite type linear differential equations, Hokkaido Math.J.17 (1988), 151-195.

[SYY97] T. Sasaki, K. Yamaguchi and M. Yoshida, On the Rigidity of Differential Systems modeled on Hermitian Symmetric Spaces and Disproofs of a Conjecture concerning Modular Interpretations of Configuration Spaces, Advanced Studies in Pure Math. 25 (1997), 318-354.

[SY98] H.Sato and A.Y.Yoshikawa, Third order ordinary differential equations and Legendre connection, J. Math. Soc. Japan, 50 (1998), 993-1013

[Tan70] N. Tanaka, On differential systems, graded Lie algebras and pseudo-groups, J.Math. Kyoto Univ.10 (1970), 1-82

[Tan79] N. Tanaka, On the equivalence problems associated with simple graded Lie algebras, Hokkaido Math. J. 8 (1979), 23-84

[Tan82] N.Tanaka, On geometry and integration of systems of second order ordinary differential equations, Proc. Symposium on Differential Geometry, 1982, pp. 194-205 (in Japanese)

[Tan85] N. Tanaka, On affine symmetric spaces and the automorphism groups of product manifolds, Hokkaido Math.J.14 (1985), 277-351.

[Tan89] N. Tanaka, Geometric theory of ordinary differential equations, Report of Grant-in-Aid for Scientific Research MESC Japan (1989).

[Yam82] K. Yamaguchi : Contact geometry of higher order, Japan.J.Math 8(1982), 109-176.

[Yam83] K. Yamaguchi : Geometrization of jet bundles, Hokkaido Math. J. 12 (1983), 27-40.

[Yam93] K. Yamaguchi : Differential Systems associated with Simple Graded Lie Algebras, Advanced Studies in Pure Mathematics 22 (1993), 413-494.

[Yam99] K. Yamaguchi : $G_{2}$-geometry of overdetermined systems of second order, Trends in Mathematics (Analysis and Geometry in Several Complex Variables) (1999), Birkhäuser, Boston, 289-314.

[YY02] K. Yamaguchi and T. Yatsui : Geometry of Higher Order Differential Equations of Finite Type associated with Symmetric Spaces, Advanced Studies in Pure Mathematics 37 (2002), 397-458.

[Yat88] T.Yatsui, On pseudo-product graded Lie algebras, Hokkaido Math. J. 17 (1988), 333-343

[Yat92] T. Yatsui, On completely reducible transitive graded Lie algebras of finite depth, Japan. J. Math. 18 (1992), 291-330.

K.Yamaguchi, Department of Mathematics,, Graduate School of Science,, Hokkaido UNIVERSITY,, SAPPORO 060-0810,, JAPAN, E-MAIL yamaguch@math.sci.hokudai.ac.jp

T. Yatsui, Department of Mathematics, Hokkaido University of Education, Asahikawa Campus,, Asahikawa 070-8261,, JAPAn, E-MAIL tomoaki@atson.asa.hokkyodai.ac.jp 